\title{
Nonlinear development of subsonic modes on compressible mixing layers: a unified strongly nonlinear critical-layer theory
}

\author{
Clifford A. Sparks \& Xuesong Wu \\ Department of Mathematics, Imperial College London, \\ 180 Queens Gate, London SW7 2AZ, UK
}

June 29, 2008

\begin{abstract}
This paper is concerned with the nonlinear instability of compressible mixing layers in the regime of small to moderate values of Mach number $M$, in which subsonic modes play a dominant role. At high Reynolds numbers of practical interest, previous studies have shown that the dominant nonlinear effect controlling the evolution of an instability wave comes from the so-called critical layer. In the incompressible limit $(M=0)$, the critical-layer dynamics are strongly nonlinear, with nonlinearity being associated with the logarithmic singularity of the velocity fluctuation (Goldstein \& Leib 1988). In contrast, in the fully compressible regime $(M=\mathcal{O}(1))$, nonlinearity is associated with a simple-pole singularity in the temperature fluctuation, and enters in a weakly nonlinear fashion (Goldstein \& Leib 1989). In this paper, we first consider a weakly compressible regime, corresponding to the distinguished scaling $M=\mathcal{O}\left(\epsilon^{1 / 4}\right)$, for which the strongly nonlinear structure persists but is affected by compressibility at leading order (where $\epsilon \ll 1$ measures the magnitude of the instability mode). A strongly nonlinear system governing the development of the vorticity and temperature perturbation is derived. It is further noted that the strength of the pole singularity is controlled by $T_{c}^{\prime}$, the mean temperature gradient at the critical level, and for typical base-flow profiles $T_{c}^{\prime}$ is small even when $M=\mathcal{O}(1)$. By treating $T_{c}^{\prime}$ as an independent parameter of $\mathcal{O}\left(\epsilon^{1 / 2}\right)$, we construct a composite strongly nonlinear theory, from which the weakly nonlinear result for $M=\mathcal{O}(1)$ can be derived as an appropriate limiting case. Thus the strongly nonlinear formulation is uniformly valid for $\mathcal{O}(1)$ Mach numbers. Numerical solutions show that this theory captures vortex roll-up process, which remains the most prominent feature of compressible mixing-layer transition. The theory offers an effective tool for investigating the nonlinear instability of mixing layers at high Reynolds numbers.
\end{abstract}

\section{1 introduction}

A mixing layer, which is formed between two parallel streams of different speeds, represents an important prototype of shear flows for studying a fundamental instability mechanism: inviscid Kelvin-Helmholtz (or Rayleigh) instability. The interest in its instability and transition to turbulence stems from their relevance to many practical applications such as combustion and other chemically reactive flows, where the mixing process is of serious technological concern (Gutmark \& Schadow 1995). The recent resurgence of research on supersonic mixing layers has in particular been driven by the plan to develop scramjet 
engines (Curran, Heiser \& Pratt 1996). The projected engine uses air-breathing technique and combustion takes place in the non-premixed mode and at supersonic speeds. Effective mixing is crucial to overcome the difficulty caused by the extremely short residence time of fuel and oxidizer in a combustor of restricted length. A compressible mixing layer provides the simplest conceptual model in which relevant mixing processes can be studied.

In typical applications, the relevant Reynolds numbers are so high that the flow is slowly evolving in the streamwise direction and local parallel-flow stability theory is deemed applicable. In the incompressible regime, linear stability analysis shows that a mixing layer without a reverse flow is convectively unstable and thus behaves like a noise amplifier (Ho \& Huerre 1984, Huerre \& Monkewitz 1990). Dominant unstable waves, excited usually by ambient unsteady disturbances, roll up under nonlinear effects to form concentrated spanwise vortices, or 'rollers', which then undergo repeated pairing (Winant \& Browand 1974, Ho \& Huerre 1984). The rollers eventually break down to small-scale motions. Yet the most striking feature is that predominantly two-dimensional large-scale roller-like structures persist in a fully turbulent state (Brown \& Roshko 1974).

Large-scale rollers as well as the associated small-scale motions directly influence mixing process. The presence of the former implies that the conventional concept of gradient diffusion for turbulent mixing may not be applicable. Broadwell \& Breidenthal (1982) demonstrated that a model taking into account large-scale structures would give significantly different predictions from diffusion models. In order to characterise mixing at the molecular level, one has to describe first the formation and structure of rolled-up vortices. That in turn requires investigating the nonlinear development of instability waves.

The classical weakly nonlinear theory (Stuart 1960), formulated for exactly parallel flows at finite Reynolds numbers, has been adapted to spatially developing mixing layers, and a certain degree of success has been achieved; see the review of Liu (1989). However, such adaption reply on ad-hoc treatments of nonlinear and non-parallel-flow effects. In order to treat systematically the delicate balance of various physical effects, it is necessary to take the Reynolds number to be asymptotically large. This has led to a self-consistent approach, known as non-equilibrium critical layer theory; for reviews see Goldstein (1994) and Cowley \& Wu (1994). A key observation is that as an initially linear instability wave propagates downstream, its growth rate diminishes due to the thickening of the shear layer, and there emerges a critical layer, i.e. a region surrounding the level at which the mean velocity $\bar{U}$ equals the phase speed of the wave, $c$ say. Outside this region, the perturbation remains linear and inviscid, but nonlinear effects become significant within the critical layer because the effective mean flow $(\bar{U}-c)$, about which linearization is made, is vanishingly small locally. In addition, the non-equilibrium effect associated with the slow modulation of the wave envelope may also appear at leading order.

Goldstein \& Leib (1988) and Goldstein \& Hultgren (1988) showed that a planar mode on an incompressible mixing layer evolves into a nonlinear stage where the critical layer is non-equilibrium, and strongly nonlinear in the sense that nonlinearity enters as a 'coefficient' of the operator governing the dynamics rather than as an inhomogeneous term of a linear system in weakly nonlinear theory (cf. Stuart 1960). Due to the strongly nonlinear nature, the disturbance in the critical-layer rolls up to form concentrated vortices, i.e. 'rollers". Capturing the crucial feature of vortex roll-up is a notable success of the strongly nonlinear critical-layer theory. Hultgren (1992) went on to construct a composite solution to take into account the non-parallel-flow effect, and demonstrated that the theory was able to make quantitatively accurate predictions.

Linear instability theory for compressible shear flows was first formulated by Lees \& 
Lin (1946), who showed that a necessary condition for a flow to be inviscidly unstable is that its velocity and temperature (density) profiles, $\bar{U}$ and $\bar{T}(\bar{\rho})$ must have a generalised inflection point, i.e. $\left(\bar{\rho} \bar{U}^{\prime}\right)^{\prime}=0$ at some transverse position $y$, where a prime denotes differentiation with respect to $y$. For a perfect gas, the condition is equivalent to

$$
\left(\bar{U}^{\prime} / \bar{T}\right)^{\prime}=0 .
$$

A significant consequence of including compressibility is that a flow that is stable in the incompressible limit may be unstable. Moreover, there may exist a multitude of instability modes at large values of a characteristic Mach number $M$. Extensive computations have been carried out to identify these modes, and major results are documented in the report by Mack (1984). Instability modes may be classified according to their propagation speeds relative to the free-stream velocity. If the relative velocity is smaller/larger than the freestream sound speed, the mode is called subsonic/supersonic. A neutral subsonic mode must have a phase speed given by $c=U\left(y_{c}\right)$, where $y_{c}$ is the point at which equation (1.1) is satisfied (Lees \& Lin 1946). Supersonic modes exist at sufficiently high Mach numbers, and its critical layer does not coincide with an inflection point.

Temporal instability of compressible mixing layers was studied by several investigators. Lessen, Fox \& Zien $(1965,1966)$ analysed the stability property of a compressible mixing layer with a velocity profile described by Lock (1951). They found that oblique modes may be more unstable than planar ones. Blumen (1970), Blumen et al. (1975) and Drazin \& Davey (1977) considered stability of a mixing layer modelled by a hyperbolic tangent (i.e. tanh) velocity profile. Three unstable modes at large Mach numbers are identified. For the case of two counter-flowing streams with equal speed, one of the modes is stationary while the other two have equal but opposite phase velocities.

A comprehensive analysis of spatial stability was carried out by Jackson \& Grosch (1989). They found that subsonic modes exist at relatively low Mach numbers. When $N$ exceeds a critical value, two groups of unstable modes come into existence, which are supersonic with respect to the slow and fast streams respectively. Planar subsonic modes are most unstable at relatively low Mach numbers. At sufficiently high Mach numbers, the most unstable modes are oblique, consistent with the earlier and subsequent temporal instability analysis (e.g. Lessen et al. 1965, 1966, Sandham \& Reynolds 1991). In general, increasing the Mach number renders the flow less unstable.

Nonlinear instability of a fully compressible mixing layer $(M=\mathcal{O}(1))$ was considered by Goldstein \& Leib (1989). They studied the evolution of a single subsonic oblique instability mode, for which the eigenfunction for the pressure is free from the logarithmic singularity. The dominant nonlinearity is associated with a pole singularity in the leading-order temperature perturbation, which makes nonlinearity appear sooner than in the incompressible case. As a result, nonlinearity operates in a weakly nonlinear fashion, that is, its effects appear successively as inhomogeneous terms at higher orders (cf. Stuart 1960). By analyzing the weakly nonlinear interaction within the critical layer, they derived an amplitude equation containing a cubic nonlinear term, which is, unlike the classical Landau equation, nonlocal because the non-equilibrium effect appears at leading order in the critical-layer equations. The analysis of Goldstein \& Leib (1989) was subsequently extended by Leib (1991) to supersonic modes.

The two existing nonlinear instability theories, a strongly nonlinear critical-layer theory in the incompressible regime $(M=0)$ and a weakly nonlinear critical-layer theory in the compressible regime with $M=\mathcal{O}(1)$, have no overlap. From the mathematical viewpoint, 
it is certainly desirable to unify them by considering a distinguished weakly compressible regime. Moreover, experiments indicate that in the moderately to fully compressible regime with $M=\mathcal{O}(1)$ (including low supersonic speeds $M>1$ ), mixing layers continue to behave in more-or-less the same manner as in the incompressible regime (Clemens \& Mungal 1995, Elliott, Samimy \& Arnette 1995). In particular, vortex roll-up remains the most significant feature of mixing layer development (Urban \& Mungal 2001, Olsen \& Dutton 2003). However, the phenomenon of vortex roll-up cannot be captured by the weakly nonlinear theory for $M=\mathcal{O}(1)$.

The aim of this paper is to develop a nonlinear theory capable of describing vortex roll-up process. This is to be achieved via two steps. As a first step, we consider a distinguished weakly compressible regime, in which the critical-layer dynamics remains strongly nonlinear but is substantially modified by compressibility. The next step involves extending the analysis to the fully compressible regime and constructing a composite theory that is uniformly valid for $M=\mathcal{O}(1)$. The proposed theoretical development is important since vortex roll-up plays a crucial role in the entrainment and mixing of the two streams (Gutmark \& Schadow 1995).

In $\S 2$, we formulate the two-dimensional instability problem for a compressible mixing layer perturbed by a planar subsonic mode. We then analyse the inviscid linear unsteady flow in the main part of the layer, and derive the asymptote of the solution as the critical level is approached, highlighting the nature of singularities, which ultimately determine the nonlinear dynamics within the critical layer.

In $\S 3$, we consider the strongly nonlinear dynamics within the non-equilibrium, viscous critical layer for the distinguished weakly compressible regime $M=\mathcal{O}\left(\epsilon^{1 / 4}\right)$, which bridges the strongly nonlinear theory in the incompressible limit $(M=0)$ and the weakly nonlinear theory for $M=\mathcal{O}(1)$, where $\epsilon$ is a measure of the magnitude of the mode. The resulting evolution system consists of an amplitude equation coupled with the equations governing the development of vorticity and temperature within the critical layer.

In $\S 4$, we extend the theory to the fully compressible regime with $M=\mathcal{O}(1)$. An extension is first made for certain velocity profiles (e.g. the tanh profile) for which $T_{c}^{\prime}=0$ even when $M=\mathcal{O}(1)$. The appropriate scaling leads to a strongly nonlinear critical layer. The vorticity and temperature evolution equations both have to be modified to take into account the effect associated with $\mathcal{O}(1)$ Mach number. The final extension is motivated by the observation that $T_{c}^{\prime}$ is numerically small for practical profiles even when $M=\mathcal{O}(1)$. We then assume that $T_{c}^{\prime}=\mathcal{O}\left(\epsilon^{1 / 2}\right)$ so as to include the compressible nonlinear effect in the strongly nonlinear structure. The final evolution system reduces to that in the incompressible limit, while on the other hand contains the amplitude equation in the weakly nonlinear theory of Goldstein \& Leib (1989), in that the latter can be derived in an appropriate limit. Thus our final system is a composite theory uniformly valid for all $0 \leqslant M \leqslant \mathcal{O}(1)$.

In $\S 5$, the strongly nonlinear system is solved numerically, and solutions are presented and discussed. A summary of main results is given in $\S 6$.

\section{Formulation \& outer flow}

\subsection{Problem specification and governing equations}

Consider two compressible streams separated by a splitter plate. The velocities of the oncoming streams are denoted by $U_{1}, U_{2}\left(U_{1}>U_{2}\right.$ say), and temperatures by $T_{1}, T_{2}$ 
respectively. Downstream of the splitter plate, a free shear layer develops. The flow will be described in terms of Cartesian coordinates $(x, y)$ with $x$ and $y$ being parallel and normal to the plate respectively, and normalized by the shear-layer thickness $l$ at the location of interest. Let $t$ be the time variable normalized by $l / U_{1}$. The non-dimensional velocity field $(u, v)$, the pressure $p$, density $\rho$, and temperature $T$ are introduced by

$$
(u, v)=\left(u^{*}, v^{*}\right) / U_{1}, \quad \rho=\rho^{*} / \rho_{1}, \quad p=\gamma M^{2} p^{*} /\left(\rho_{1} U_{1}^{2}\right), \quad T=T^{*} / T_{1},
$$

where ${ }^{*}$ signifies dimensional quantities, $\rho_{1}$ the density of the fast stream, $\gamma$ is the ratio of specific heats $\gamma=c_{p} / c_{v}$. We define Mach number $M$ and Reynolds number $R$ as

$$
M=U_{1} / a_{1}, \quad R=\rho_{1} U_{1} l / \mu_{1},
$$

with $\mu_{1}$ being the viscosity coefficient in the fast stream. We shall assume $R \gg 1$. Two further parameters, which affect the mean profiles and hence instability, are the velocity and temperature ratios, defined as

$$
\beta_{U}=U_{2} / U_{1}, \quad \beta_{T}=T_{2} / T_{1}
$$

On assuming the fluid to be a perfect gas, the non-dimensional compressible NavierStokes equations in two-dimensions can be written as (Schlichting \& Gersten 2000)

$$
\begin{gathered}
T D p-p D T+p T\left(\frac{\partial u}{\partial x}+\frac{\partial v}{\partial y}\right)=0, \\
p D u=-\frac{T}{\gamma M^{2}} \frac{\partial p}{\partial x}+\frac{T}{R}\left\{\frac{2}{3} \frac{\partial}{\partial x}\left[\mu\left(2 \frac{\partial u}{\partial x}-\frac{\partial v}{\partial y}\right)\right]+\frac{\partial}{\partial y}\left[\mu\left(\frac{\partial u}{\partial y}+\frac{\partial v}{\partial x}\right)\right]\right\}, \\
p D v=-\frac{T}{\gamma M^{2}} \frac{\partial p}{\partial y}+\frac{T}{R}\left\{\frac{2}{3} \frac{\partial}{\partial y}\left[\mu\left(2 \frac{\partial v}{\partial y}-\frac{\partial u}{\partial x}\right)\right]+\frac{\partial}{\partial x}\left[\mu\left(\frac{\partial u}{\partial y}+\frac{\partial v}{\partial x}\right)\right]\right\}, \\
p D T=\frac{(\gamma-1)}{\gamma} T D p+\frac{T}{\operatorname{Pr} R}\left\{\frac{\partial}{\partial x}\left(\mu \frac{\partial T}{\partial x}\right)+\frac{\partial}{\partial y}\left(\mu \frac{\partial T}{\partial y}\right)\right\} \\
+\frac{(\gamma-1) M^{2}}{R} T \mu\left\{\frac{4}{3}\left[\left(\frac{\partial u}{\partial x}\right)^{2}+\left(\frac{\partial v}{\partial y}\right)^{2}-\frac{\partial u}{\partial x} \frac{\partial v}{\partial y}\right]+\left(\frac{\partial v}{\partial x}+\frac{\partial u}{\partial y}\right)^{2}\right\},
\end{gathered}
$$

supplemented by the dimensionless state equation

$$
p=\rho T
$$

In the above, the material derivative $D \equiv \partial / \partial t+u \partial / \partial x+v \partial / \partial y$, and $\operatorname{Pr}=c_{p} \mu_{1} / k$ is the Prandtl number $\operatorname{Pr}$ with $k$ being the thermal diffusivity. The viscosity $\mu$ is assumed to be a function of the temperature $T$ only, i.e. $\mu=\mu(T)$.

\subsection{The base flow}

Except in the vicinity of the trailing edge of the splitter plate, the base flow is described by the classical boundary layer theory, according to which, the solution expands as

$$
\left(U_{B}, V_{B}, P_{B}, \rho_{B}, T_{B}, \mu_{B}\right)=\left(\bar{U}\left(\mathcal{X}_{2}, y\right), R^{-1} \bar{V}, 1, \bar{\rho}, \bar{T}, \bar{\mu}\right)+\mathcal{O}\left(R^{-1}\right),
$$


where the subscript $B$ denotes basic flow quantities, and $(\bar{U}, \bar{V}, \bar{\rho}, \bar{T}, \bar{\mu})$ are functions of $y$ and $\mathcal{X}_{2}=x / R$, a slow streamwise variable. Substituting (2.8) into (2.3)-(2.7) yields equations for $\bar{U}, \bar{V}$ and $\bar{T}$, which can be converted into a form analogous to the incompressible case by using the Howarth-Dorodnitsyn transformation (Stewartson 1964, p29)

$$
\eta=\int_{0}^{y} \bar{\rho}\left(\mathcal{X}_{2}, \tilde{y}\right) \mathrm{d} \tilde{y}, \quad \widehat{V}\left(\mathcal{X}_{2}, \eta\right)=\bar{\rho} \bar{V}+\bar{U} \int_{0}^{y} \bar{\rho}_{\mathcal{X}_{2}}\left(\mathcal{X}_{2}, \tilde{y}\right) \mathrm{d} \tilde{y} .
$$

Consideration of a similarity solution of the form

$$
\bar{U}=f^{\prime}(q), \quad \widehat{V}=-\left(2 \mathcal{X}_{2}\right)^{-1 / 2}\left(f(q)-q f^{\prime}(q)\right), \quad \bar{T}=\bar{T}(q), \quad q=\eta /\left(2 \mathcal{X}_{2}\right)^{1 / 2}
$$

leads to a pair of coupled ordinary differential equations for $f$ and $\bar{T}$,

$$
\left(\frac{\bar{\mu}}{\bar{T}} f^{\prime \prime}\right)^{\prime}+f f^{\prime \prime}=0, \quad \frac{1}{P_{r}}\left(\frac{\bar{\mu}}{\bar{T}} \bar{T}^{\prime}\right)^{\prime}+f \bar{T}^{\prime}+(\gamma-1) M^{2}\left(\frac{\bar{\mu}}{\bar{T}}\right)\left(f^{\prime \prime}\right)^{2}=0,
$$

where the primes denote differentiation with respect to the similarity variable $q$. Equations in (2.11) are subjected to the boundary conditions

$$
\left(f^{\prime}, \bar{T}\right) \rightarrow(1,1) \text { as } \eta \rightarrow+\infty, \quad\left(f^{\prime}, \bar{T}\right) \rightarrow\left(\beta_{U}, \beta_{T}\right) \text { as } \eta \rightarrow-\infty, \quad f(0)=0 .
$$

Under the assumption of unity Prandtl number, the so-called Crocco relation,

$$
\bar{T}=1-\frac{\left(1-\beta_{T}\right)}{\left(1-\beta_{U}\right)}(1-\bar{U})-\frac{1}{2}(\gamma-1) M^{2}\left[(\bar{U}-1)\left(\bar{U}-\beta_{U}\right)\right]
$$

can be derived from (2.11) (Grosch \& Jackson 1991), where $\beta_{U}$ and $\beta_{T}$ are defined by (2.2). This relation holds for an arbitrary viscosity law $\bar{\mu}=\bar{\mu}(\bar{T})$ (Stewartson 1964, p44).

The solution of (2.11) $-(2.12)$ is to be found numerically. For the Chapman law $\bar{\mu}=\bar{T}$, the two equations in (2.11) decouple, with the first reducing to the Blasius equation, and the solution is equivalent to the Lock (1951) profile with uniform density and viscosity.

To the order of approximation of the subsequent stability analysis, it suffices to specify the velocity and temperature profiles only at the location of interest (Leib 1991). Therefore, for brevity the slow variable $\mathcal{X}_{2}$ will be suppressed hereafter.

As a common practice in mixing-layer instability studies, most of our subsequent numerical calculations will focus on the tanh profile,

$$
\bar{U}=\frac{1}{2}\left(1+\beta_{U}+\left(1-\beta_{U}\right) \tanh (\eta+s)\right),
$$

where $s$ is a constant, representing a shift of the coordinate so that the critical level is located at $\eta=0$. The extent to which (2.14) approximates the more realistic profile (2.11) is examined in Sparks (2006).

\subsection{Perturbation and the outer flow}

To study the stability, we perturb the basic flow. The perturbed flow can be written as

$$
(u, v, p, T, \mu)=\left(U_{B}, V_{B}, P_{B}, T_{B}, \mu_{B}\right)+\epsilon\left(\tilde{u}, \tilde{v}, \gamma M^{2} \tilde{p}, \tilde{T}, \tilde{\mu}\right),
$$

where the perturbation is assumed to be an instability mode, whose overall magnitude is small, i.e. $\epsilon \ll 1$. As a mode propagates downstream, its local linear growth rate 
gradually decreases due to the thickening of the shear layer, and would become neutral at a streamwise location $x_{n}$, known as neutral position. Without loss of generality, the origin of the coordinate is taken to be $x_{n}$. Close to $x_{n}$, a critical layer emerges, where nonlinear effects may become important first, whilst the unsteady flow outside the critical layer remains linear (Goldstein \& Leib 1988). The analysis must therefore be performed for two distinct regions: a linear inviscid outer layer, and a nonlinear viscous inner region, i.e. the critical layer. We would like to remind the reader that the analysis in this paper is generally independent of base-flow profiles; the specific profiles and Crocco's relation are used primarily for scaling arguments.

The perturbation is of the travelling-wave form. In the main part of the shear layer,

$$
\tilde{q}=\left(A(\mathcal{X}, \mathcal{T}) \tilde{q}_{0}(y) \mathrm{e}^{\mathrm{i} \zeta}+\text { c.c. }\right)+\Delta \tilde{q}_{1}(\mathcal{X}, \mathcal{T}, y, \zeta)+O\left(\Delta^{2}, \epsilon\right)+\cdots,
$$

where $\tilde{q}$ represents any of $\tilde{u}, \tilde{v}, \tilde{p}$ or $\widetilde{T}$. The variable $\zeta$ is defined as

$$
\zeta=\alpha x-\omega t
$$

and it represents a moving coordinate. Both the wavenumber $\alpha$ and frequency $\omega(=\alpha c)$ are real in the vicinity of $x_{n}$, where the disturbance evolves nonlinearly. The nonlinear development can be described by introducing an amplitude function $A(\mathcal{X}, \mathcal{T})$, which depends on the slow streamwise and temporal variables

$$
\mathcal{X}=\Delta x, \quad \mathcal{T}=\Delta t
$$

where the time/length scale on which the amplitude evolves, $\Delta^{-1}$, is to be determined later. Substitution of (2.16)-(2.18) into (2.3)-(2.6) yields at $\mathcal{O}(\epsilon)$ the equations

$$
\begin{gathered}
\mathrm{i} \alpha(\bar{U}-c) M^{2} \tilde{p}_{0}+\mathrm{i} \alpha \tilde{u}_{0}+\tilde{v}_{0}^{\prime}=0, \\
\mathrm{i} \alpha(\bar{U}-c) \tilde{u}_{0}+\bar{U}^{\prime} \tilde{v}_{0}+\mathrm{i} \alpha \bar{T} \tilde{p}_{0}=0, \\
\mathrm{i} \alpha(\bar{U}-c) \tilde{v}_{0}+\bar{T} \tilde{p}_{0}^{\prime}=0, \\
\mathrm{i} \alpha(\bar{U}-c) \widetilde{T}_{0}+\bar{T}^{\prime} \tilde{v}_{0}+(\gamma-1) \bar{T}\left(\mathrm{i} \alpha \tilde{u}_{0}+\tilde{v}_{0}^{\prime}\right)=0 .
\end{gathered}
$$

On eliminating $\widetilde{T}_{0}, \tilde{u}_{0}$ and $\tilde{v}_{0}$ from $(2.19)-(2.22)$, we can derive the equation

$$
\mathcal{L} \tilde{p}_{0}=0,
$$

for the pressure $\tilde{p}_{0}$, where $\mathcal{L}$ is the compressible Rayleigh operator, defined as

$$
\mathcal{L} \equiv \frac{\partial^{2}}{\partial y^{2}}+\left[\frac{\bar{T}^{\prime}}{\bar{T}}-\frac{2 \bar{U}^{\prime}}{(\bar{U}-c)}\right] \frac{\partial}{\partial y}+\alpha^{2}\left[\frac{M^{2}(\bar{U}-c)^{2}}{\bar{T}}-1\right]
$$

For subsonic modes of interest in this study, the boundary conditions for (2.23) are

$$
\tilde{p}_{0} \rightarrow 0 \quad \text { as } \quad y \rightarrow \pm \infty
$$

For a subsonic mode, its critical level $y_{c}$ coincides with the generalised inflection point (Lees \& Lin 1946), so that

$$
T_{c}^{\prime} / T_{c}=U_{c}^{\prime \prime} / U_{c}^{\prime}
$$


holds, where subscript ' $c$ ' denotes the value of the mean-flow quantity at $y_{c}$. For $y$ close to $y_{c}=0$, one can find the local solution to (2.23) (cf. Goldstein \& Leib 1989),

$$
\tilde{p}_{0}=\frac{U_{c}^{\prime}}{T_{c}}\left[\pi_{1}(y ; \alpha)+\frac{1}{3} \alpha^{2}\left(b_{1}+\frac{1}{2} \frac{U_{c}^{\prime \prime}}{U_{c}^{\prime}}\right) \pi_{2}(y ; \alpha)\right],
$$

using Fröbenius method, where $b_{1}$ is a constant to be determined by solving the global boundary-value problem (2.23)-(2.24), and

$$
\pi_{1}(y ; \alpha)=1-\frac{1}{2} \alpha^{2} y^{2}+a_{4} y^{4}+\mathcal{O}\left(y^{6}\right) \cdots, \quad \pi_{2}(y ; \alpha)=y^{3}+\mathcal{O}\left(y^{5}\right)+\cdots,
$$

with

$$
a_{4}=\frac{1}{4} \alpha^{2}\left[\frac{T_{c}^{\prime \prime}}{T_{c}}-\frac{2}{3} \frac{U_{c}^{\prime \prime \prime}}{U_{c}^{\prime}}-\frac{1}{2}\left(\frac{T_{c}^{\prime}}{T_{c}}\right)^{2}-\frac{1}{2} \alpha^{2}-\frac{U_{c}^{\prime 2} M^{2}}{T_{c}}\right] .
$$

Inserting (2.26) into (2.19)-(2.22), we find asymptotic solutions for $\tilde{u}_{0}, \tilde{v}_{0}$ and $\widetilde{T}_{0}$,

$$
\begin{gathered}
\tilde{u}_{0}=-b_{1}-e_{1} y+\mathcal{O}\left(y^{2}\right), \\
\tilde{v}_{0}=-\mathrm{i} \alpha+\mathrm{i} \alpha b_{1} y+\frac{1}{2} \mathrm{i} \alpha\left(e_{1}-M^{2} U_{c}^{\prime 2} / T_{c}\right) y^{2}+\mathcal{O}\left(y^{3}\right), \\
\widetilde{T}_{0}=\frac{T_{c}^{\prime}}{U_{c}^{\prime}} \frac{1}{y}+\frac{1}{U_{c}^{\prime}}\left(T_{c}^{\prime \prime}-T_{c}^{\prime} b_{1}-\frac{1}{2} \frac{T_{c}^{\prime 2}}{T_{c}}+(\gamma-1) M^{2} U_{c}^{\prime 2}\right)+\mathcal{O}(y) .
\end{gathered}
$$

where $e_{1}=T_{c}^{\prime \prime} / T_{c}+b_{1} T_{c}^{\prime} / T_{c}-U_{c}^{\prime \prime \prime} / U_{c}^{\prime}-\alpha^{2}-M^{2} U_{c}^{\prime 2} / T_{c}$. Evidently, the leading-order pressure $\tilde{p}_{0}$, and velocities $\tilde{u}_{0}$ and $\tilde{v}_{0}$, are all regular, but the leading-order temperature $\tilde{T}_{0}$ has a pole singularity if $T_{c}^{\prime} \neq 0$.

The second term in expansion (2.16) can be expressed as

$$
\tilde{q}_{1}=\sum_{\substack{m=-\infty \\ m \neq 0}}^{\infty} \tilde{q}_{1}^{(m)}(\mathcal{X}, \mathcal{T}, y) \mathrm{e}^{\mathrm{i} m \zeta} \quad\left(\text { with } \quad \tilde{q}_{1}^{(-m)}=\tilde{q}_{1}^{(m) *}\right)
$$

where $^{*}$ represents complex conjugate. Substitution of (2.16)-(2.18) into (2.3)-(2.6) yields, at $\mathcal{O}(\epsilon \Delta)$, equations $(2.19)-(2.22)$ for the harmonics $\tilde{q}_{1}^{(m)}(m \neq-1,0,1)$, with $\alpha$ being replaced by $m \alpha$. The pressure harmonics $\tilde{p}_{1}^{(m)}(m \neq-1,0,1)$ are governed by $(2.23)$ with $\alpha \rightarrow m \alpha$. The solution can be expressed as

$$
\tilde{p}_{1}^{(m)}=d_{1}^{(m)}(\mathcal{X}, \mathcal{T}) \tilde{\pi}_{1}(y ; m \alpha)+d_{2}^{(m) \pm}(\mathcal{X}, \mathcal{T}) \tilde{\pi}_{2}(y ; m \alpha),
$$

where $d_{1}^{(m)}$ and $d_{2}^{(m) \pm}$ are arbitrary functions of $\mathcal{X}$ and $\mathcal{T}$.

The fundamental component $(m=1)$ at $\mathcal{O}(\epsilon \Delta),\left(\tilde{u}_{1}^{(1)}, \tilde{v}_{1}^{(1)}, \tilde{p}_{1}^{(1)}, \widetilde{T}_{1}^{(1)}\right)$, satisfies the inhomogeneous version of (2.19-2.22). Elimination of $\tilde{u}_{1}^{(1)}, \tilde{v}_{1}^{(1)}$ and $\tilde{T}_{1}^{(1)}$ yields an inhomogeneous Rayleigh equation for $\tilde{p}_{1}^{(1)}$,

$$
\mathcal{L} \tilde{p}_{1}^{(1)}=\frac{2 \mathrm{i}}{\alpha}\left(A_{\mathcal{T}}+c A_{\mathcal{X}}\right)\left[\frac{\bar{U}^{\prime}}{(\bar{U}-c)^{2}} \tilde{p}_{0}^{\prime}+\frac{\alpha^{2} M^{2}(\bar{U}-c)}{\bar{T}} \tilde{p}_{0}\right]+2 \mathrm{i} \alpha\left[\frac{M^{2}(\bar{U}-c)^{2}}{\bar{T}}-1\right] A_{\mathcal{X}} \tilde{p}_{0}
$$

where the subscripts $\mathcal{T}$ and $\mathcal{X}$ denote partial differentiations. The solution, valid near $y_{c}=0$, is given by

$$
\tilde{p}_{1}^{(1)}=\tilde{p}_{1 p s}^{(1)}+d_{1}(\mathcal{X}, \mathcal{T}) \pi_{1}(y ; \alpha)+d_{2}^{ \pm}(\mathcal{X}, \mathcal{T}) \pi_{2}(y ; \alpha),
$$


where $\tilde{p}_{1 p s}^{(1)}$ denotes a particular solution of (2.33), given by

$$
\begin{gathered}
\tilde{p}_{1 p s}^{(1)}=\frac{\mathrm{i} \alpha}{T_{c}}\left(A_{\mathcal{T}}+c A_{\mathcal{X}}\right) y+\frac{\mathrm{i} \alpha}{T_{c}}\left[U_{c}^{\prime} A_{\mathcal{X}}-\left(A_{\mathcal{T}}+c A_{\mathcal{X}}\right)\left(b_{1}+\frac{1}{2} \frac{U_{c}^{\prime \prime}}{U_{c}^{\prime}}\right)\right] y^{2} \\
+\frac{1}{3} \frac{\mathrm{i} \alpha}{T_{c}}\left(A_{\mathcal{T}}+c A_{\mathcal{X}}\right)\left(\frac{T_{c}^{\prime \prime}}{T_{c}}-\frac{U_{c}^{\prime \prime \prime}}{U_{c}^{\prime}}\right) y^{3} \ln |y|+\mathcal{O}\left(y^{4} \ln |y|\right) .
\end{gathered}
$$

The solutions for $\tilde{u}_{1}^{(1)}, \tilde{v}_{1}^{(1)}$ and $\widetilde{T}_{1}^{(1)}$ are found as

$$
\begin{gathered}
\tilde{u}_{1}^{(1)}=\frac{\mathrm{i}}{\alpha U_{c}^{\prime}}\left(\frac{U_{c}^{\prime \prime \prime}}{U_{c}^{\prime}}-\frac{T_{c}^{\prime \prime}}{T_{c}}\right)\left(A_{\mathcal{T}}+c A_{\mathcal{X}}\right) \ln |y|+\left(e_{2}-\frac{3 T_{c}}{U_{c}^{\prime} \alpha^{2}} d_{2}^{ \pm}\right)+\mathcal{O}(y \ln |y|), \\
\tilde{v}_{1}^{(1)}=\frac{1}{U_{c}^{\prime}}\left(b_{1}\left(A_{\mathcal{T}}+c A_{\mathcal{X}}\right)-U_{c}^{\prime} A_{\mathcal{X}}-\mathrm{i} \alpha T_{c} d_{1}\right)+\frac{1}{U_{c}^{\prime}}\left(\frac{U_{c}^{\prime \prime \prime}}{U_{c}^{\prime}}-\frac{T_{c}^{\prime \prime}}{T_{c}}\right)\left(A_{\mathcal{T}}+c A_{\mathcal{X}}\right) y \ln |y|+\mathcal{O}(y), \\
\widetilde{T}_{1}^{(1)}=\frac{\mathrm{i} \alpha}{U_{c}^{\prime 2}}\left\{\left(A_{\mathcal{T}}+c A_{\mathcal{X}}\right) \frac{T_{c}^{\prime}}{y^{2}}+\left[\left(\frac{T_{c}^{\prime \prime}}{T_{c}}-\frac{T_{c}^{\prime 2}}{T_{c}^{2}}\right)\left(A_{\mathcal{T}}+c A_{\mathcal{X}}\right)-\mathrm{i} \alpha T_{c}^{\prime} d_{1}\right] \frac{T_{c}}{y}\right\}+\mathcal{O}(\ln |y|) ;
\end{gathered}
$$

here $e_{2}$ in (2.36) denotes a function of $\mathcal{X}$ and $\mathcal{T}$ having the same value above and below the critical level $y_{c}$.

In order for (2.33) to have a solution also satisfying the boundary conditions, a solvability condition must be imposed. This can be derived by multiplying $(2.33)$ by $\tilde{p}_{0} \bar{T} /(\bar{U}-c)^{2}$ and integrating from $y=-\infty$ to $y=+\infty$, leading to

$$
-\frac{3}{U_{c}^{\prime}}\left(d_{2}^{+}-d_{2}^{-}\right)=2 \mathrm{i}\left(c J_{1} \frac{\partial A}{\partial \mathcal{X}}+J_{2} \frac{\partial A}{\partial \mathcal{T}}\right)
$$

where we have put $J_{1}=J_{2}+(\alpha / c) J_{3}$,

$$
J_{2}=\frac{1}{\alpha} \int_{-\infty}^{+\infty} \frac{\bar{T} \bar{U}^{\prime} \tilde{p}_{0} \tilde{p}_{0}^{\prime}+\alpha^{2} M^{2}(\bar{U}-c)^{3} \tilde{p}_{0}^{2}}{(\bar{U}-c)^{4}} \mathrm{~d} y, \quad J_{3}=\int_{-\infty}^{+\infty} \frac{M^{2}(\bar{U}-c)^{2}-\bar{T}}{(\bar{U}-c)^{2}} \tilde{p}_{0}^{2} \mathrm{~d} y
$$

Here $f_{-\infty}^{\infty}$ denotes the finite part in the sense of Hadamard. The difference $\left(d_{2}^{+}-d_{2}^{-}\right)$is related to the jump in the streamwise velocity (see (2.36)) across the critical layer

$$
\tilde{u}_{1}^{(1)}(y=0+)-\tilde{u}_{1}^{(1)}(y=0-)=-\frac{3 T_{c}}{\alpha^{2} U_{c}^{\prime}}\left(d_{2}^{+}-d_{2}^{-}\right) .
$$

The velocity jump will be obtained by analysing the critical layer, which involves seeking the inner critical-layer solution and then matching it to the outer solution.

The reader is reminded that in the outer flow we have treated $M=\mathcal{O}(1)$. The solution is of course valid for $M \ll \mathcal{O}(1)$. Note that when $\beta_{T}=1$ and $M=0, T_{c}^{\prime}=0$. In this case, the critical-layer nonlinearity is associated with the logarithmic term in $\tilde{u}_{1}^{(1)}$ (see (2.36)), and the dynamics is governed by the strongly nonlinear theory of Goldstein \& Leib (1988). If $\beta_{T} \neq 1$ and/or $M=\mathcal{O}(1)$, then $T_{c}^{\prime}$ is of $\mathcal{O}(1)$ in general, and the criticallayer nonlinearity is associated with the pole in $\widetilde{T}_{0}$ (see $(2.30)$ ), and so nonlinear effects operate in a weakly nonlinear fashion (Goldstein \& Leib 1989, Leib 1991).

In the next section, we will consider the distinguished weakly compressible regime $M \ll \mathcal{O}(1)$, in which both singularities contribute to the nonlinear effect, so that the above two theories can be unified. 


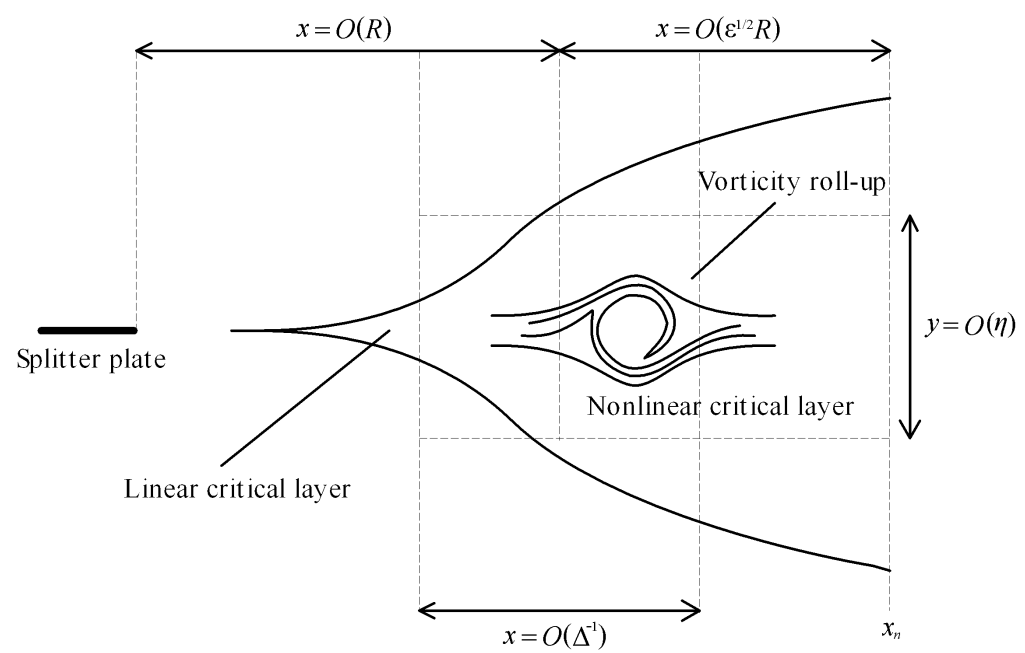

Figure 1: Sketch of the flow structure (after Goldstein \& Leib (1988)). An instability wave with an amplitude of $\mathcal{O}(\epsilon)$ enters the nonlinear critical layer regime at a distance of $\mathcal{O}\left(\epsilon^{1 / 2} R\right)$ upstream of the neutral position $x_{n}$. The strongly nonlinear evolution occurs on the length scale of order $\Delta^{-1} \sim \epsilon^{-1 / 2}$.

\section{Nonlinear critical layer in the weakly compressible regime}

\subsection{Scalings}

The singular nature of the outer solution at $y_{c}$ implies that either or both of viscous and non-equilibrium effects, which are negligible in the main part of the flow, have to be included within the critical layer in order to smooth out the singularity. More importantly, nonlinearity within the critical layer controls the overall development of the disturbance.

In the weakly compressible regime $M \ll 1$, it is anticipated that the strongly nonlinear structure (see figure 1) should remain essentially intact so that the critical layer has a width of $\mathcal{O}\left(\epsilon^{1 / 2}\right)$ (Goldstein \& Leib 1988). Thus the appropriate inner variable is defined as

$$
Y=y / \epsilon^{1 / 2}=\mathcal{O}(1) .
$$

The non-equilibrium and viscous effects both appear at leading order if

$$
\Delta=\epsilon^{1 / 2}, \quad R^{-1}=\Lambda \epsilon^{3 / 2},
$$

where $\Lambda$ is an $\mathcal{O}(1)$ parameter (Goldstein \& Hultgren 1988).

The distinguished scaling is the one such that the nonlinearity effects of the velocity and temperature perturbations are comparable. An estimate of the contribution from the nonlinear interaction associated with the temperature fluctuation shows that this occurs when $T_{c}^{\prime}=\mathrm{O}\left(\epsilon^{1 / 2}\right)$. It follows from (2.13) that we require $M \sim \epsilon^{1 / 4}$, and the temperature difference across the shear layer $\left(1-\beta_{T}\right) \sim M^{2}$. Thus we write

$$
M=\mathcal{M} \epsilon^{1 / 4}, \quad 1-\beta_{T}=\hat{\beta} M^{2},
$$

where $\mathcal{M}$ and $\hat{\beta}$ are $\mathcal{O}(1)$ parameters. 


\subsection{Critical-layer analysis}

With the scaling relations (3.2)-(3.3), rewriting the outer asymptotic solution in terms of $Y$ suggests that the solution for the perturbation in the critical layer expands as

$$
\tilde{q}=\bar{q}_{0}+\epsilon^{1 / 2}\left(\bar{q}_{1}+\left(\ln \epsilon^{1 / 2}\right) \bar{q}_{1 L}\right)+\epsilon\left(\bar{q}_{2}+\left(\ln \epsilon^{1 / 2}\right) \bar{q}_{2 L}\right)+\cdots,
$$

where $\tilde{q}$ represents any of $\tilde{p}, \tilde{u}, \tilde{v}$, or $\widetilde{T}$, and $\bar{q}_{i}=\bar{q}_{i}(\zeta, \mathcal{X}, \mathcal{T}, Y)$. Near $y_{c}$, the basic temperature and velocity profiles are approximated as

$$
\begin{gathered}
\bar{T}=T_{c}+M^{2}\left(\epsilon^{1 / 2} T_{c \mathrm{M}}^{\prime} Y+\frac{1}{2} \epsilon T_{c \mathrm{M}}^{\prime \prime} Y^{2}+\cdots\right), \\
\bar{U}=c+\epsilon^{1 / 2} U_{c}^{\prime} Y+\frac{1}{2} M^{2} \epsilon U_{c \mathrm{M}}^{\prime \prime} Y^{2}+\frac{1}{6} \epsilon^{3 / 2} U_{c}^{\prime \prime \prime} Y^{3}+\cdots,
\end{gathered}
$$

where $T_{c \mathrm{M}}^{\prime}=T_{c}^{\prime} / M^{2}=\mathcal{O}(1)$ and $U_{c \mathrm{M}}^{\prime \prime}=U_{c}^{\prime \prime} / M^{2}=\mathcal{O}(1)$. Substituting (3.4) and (3.5)-(3.6) into $(2.3)-(2.6)$, we obtain the solution for $\bar{v}_{0}$ and $\bar{p}_{0}$,

$$
\bar{v}_{0}=-\mathrm{i} \alpha A \mathrm{e}^{\mathrm{i} \zeta}+\text { c.c. }, \quad \bar{p}_{0}=\left(U_{c}^{\prime} / T_{c}\right) A \mathrm{e}^{\mathrm{i} \zeta}+\text { c.c. },
$$

from the leading-order continuity and momentum equations, while on relabelling $\bar{T}_{0} \equiv \widehat{T}$, the leading-order energy equation can be written as

$$
\mathcal{L}_{N} \widehat{T}=\mathcal{M}^{2} T_{c M}^{\prime}\left(\mathrm{i} \alpha A \mathrm{e}^{\mathrm{i} \zeta}+\text { c.c. }\right),
$$

where

$$
\mathcal{L}_{N} \equiv \alpha U_{c}^{\prime} Y \frac{\partial}{\partial \zeta}+\frac{\partial}{\partial \mathcal{T}}+c \frac{\partial}{\partial \mathcal{X}}-\left(\mathrm{i} \alpha A \mathrm{e}^{\mathrm{i} \zeta}+\text { c.c. }\right) \frac{\partial}{\partial Y}-\Lambda T_{c} \mu_{c} \frac{\partial^{2}}{\partial Y^{2}} .
$$

Substitution of (3.4) and (3.5)-(3.6) into (2.3)-(2.6), yields, at the second order,

$$
\alpha \bar{u}_{0 \zeta}+\bar{v}_{1 Y}=0, \quad \mathcal{L}_{N} \bar{u}_{0}+U_{c}^{\prime} \bar{v}_{1}=-\alpha T_{c} \bar{p}_{1 \zeta}-T_{c} \bar{p}_{0 \mathcal{X}}, \quad \bar{p}_{1 Y}=0 .
$$

The third equation implies that $\bar{p}_{1}$ is independent of $Y$ so that it can be expressed as

$$
\bar{p}_{1}=\sum_{\substack{m=-\infty \\ m \neq 0}}^{\infty} d_{1}^{(m)}(\mathcal{X}, \mathcal{T}) \mathrm{e}^{\mathrm{i} m \zeta}
$$

after matching with the outer solution (2.32) and (2.34). Elimination of $\bar{v}_{1}$ among (3.10) shows that $\mathcal{L}_{\mathcal{N}} \bar{u}_{0 Y}=0$. The solution which matches with the outer solution (2.28) is

$$
\bar{u}_{0}=-b_{1} A \mathrm{e}^{\mathrm{i} \zeta}+\text { c.c. }
$$

Finally, inserting (3.4) and (3.5)-(3.6) into (2.3)-(2.6), we obtain, at the third order,

$$
\begin{gathered}
-\mathcal{M}^{2} T_{c \mathrm{M}}^{\prime} \bar{v}_{0}-\left(\alpha U_{c}^{\prime} Y \widehat{T}_{\zeta}+\widehat{T}_{\mathcal{T}}+c \widehat{T}_{\mathcal{X}}+\bar{v}_{0} \widehat{T}_{Y}\right)+\alpha T_{c}\left(\bar{u}_{1 \zeta}+\bar{u}_{0 \mathcal{X}}+\bar{v}_{2 Y}\right)=0 \\
\mathcal{L}_{N} \bar{u}_{1}+Y U_{c}^{\prime} \bar{u}_{0 \mathcal{X}_{1}}+\alpha \bar{u}_{0} \bar{u}_{0 \zeta}+U_{c}^{\prime} \bar{v}_{2}+\frac{1}{2} U_{c}^{\prime \prime \prime} Y^{2} \bar{v}_{0}+\mathcal{M}^{2} U_{c \mathrm{M}}^{\prime \prime} Y \bar{v}_{0} \\
=-\alpha T_{c} \bar{p}_{2 \zeta}-T_{c} \bar{p}_{1 \mathcal{X}}-\alpha \mathcal{M}^{2} T_{c \mathrm{M}}^{\prime} Y \bar{p}_{0 \zeta}-\alpha \widehat{T} \bar{p}_{0 \zeta}+\Lambda T_{c} U_{c}^{\prime} \mu_{c}^{\prime} \widehat{T}_{Y} \\
\alpha U_{c}^{\prime} Y \bar{v}_{0 \zeta}+\bar{v}_{0 \mathcal{T}}+c \bar{v}_{0 \mathcal{X}}=-T_{c} \bar{p}_{2 Y}
\end{gathered}
$$

where $\mu_{c}^{\prime}=\mu_{c}^{\prime}\left(T_{c}\right)$. Differentiating (3.14) with respect to $Y$, followed by using (3.13) to eliminate $\left(\alpha \bar{u}_{1 \zeta}+\bar{v}_{2 Y}\right)$, then taking the $\zeta$-derivative of (3.15) to eliminate $\bar{p}_{2}$, we obtain

$$
\mathcal{L}_{N} \bar{u}_{1 Y}+\Lambda U_{c}^{\prime}\left(\mu_{c}-T_{c} \mu_{c}^{\prime}\right) \widehat{T}_{Y Y}=\frac{U_{c}^{\prime}}{T_{c}} \bar{v}_{0} \widehat{T}_{Y}-U_{c}^{\prime \prime \prime} Y \bar{v}_{0}+\alpha^{2} U_{c}^{\prime} Y \bar{v}_{0 \zeta \zeta}+\alpha \bar{v}_{0 \mathcal{T} \zeta}+\alpha c \bar{v}_{0 \mathcal{X} \zeta}
$$


where use has been made of (2.25), (3.7) and (3.8). Now inserting the substitution

$$
\bar{u}_{1 Y}=\widehat{Q}+\left[\left(\alpha^{2}+U_{c}^{\prime \prime \prime} / U_{c}^{\prime}\right) A \mathrm{e}^{\mathrm{i} \zeta}+\text { c.c. }\right]
$$

into (3.16) simplifies it to

$$
\mathcal{L}_{N} \widehat{Q}=\frac{U_{c}^{\prime}}{T_{c}}\left(-\mathrm{i} \alpha A \mathrm{e}^{\mathrm{i} \zeta}+\text { c.c. }\right) \widehat{T}_{Y}-\frac{U_{c}^{\prime \prime \prime}}{U_{c}^{\prime}}\left\{\left(A_{\mathcal{T}}+c A_{\mathcal{X}}\right) \mathrm{e}^{\mathrm{i} \zeta}+\text { c.c. }\right\}-\Lambda U_{c}^{\prime} \mu_{c} \sigma_{c} \widehat{T}_{Y Y},
$$

where we have put

$$
\sigma_{c}=1-T_{c} \mu_{c}^{\prime} / \mu_{c}
$$

The $\mathcal{O}\left(\epsilon^{3 / 2}\right)$ streamwise velocity jump in the outer solution (2.40) for the fundamental component must match with that in the inner solution. This can be expressed as

$$
-\frac{3 T_{c}}{\alpha^{2} U_{c}^{\prime}}\left(d_{2}^{+}-d_{2}^{-}\right)=\int_{-\infty}^{\infty}\left\{\frac{1}{2 \pi} \int_{0}^{2 \pi} \widehat{Q} \mathrm{e}^{-\mathrm{i} \zeta} \mathrm{d} \zeta\right\} \mathrm{d} Y .
$$

Similarly, for $m \neq-1,0,1$, matching requires

$$
-\frac{3 T_{c}}{(m \alpha)^{2} U_{c}^{\prime}}\left(d_{2}^{(m)+}-d_{2}^{(m)-}\right)=\frac{1}{2 \pi} \int_{-\infty}^{\infty} \int_{0}^{2 \pi} \widehat{Q} \mathrm{e}^{-\mathrm{i} m \zeta} \mathrm{d} \zeta \mathrm{d} Y .
$$

Combining (3.19) with the solvability condition (2.38), we obtain the amplitude equation

$$
f_{-\infty}^{\infty} \int_{0}^{2 \pi} \widehat{Q}, \mathrm{e}^{-\mathrm{i} \zeta} \mathrm{d} \zeta \mathrm{d} Y=4 \pi \mathrm{i} \frac{T_{c}}{\alpha^{2}}\left(c J_{1} \frac{\partial A}{\partial \mathcal{X}}+J_{2} \frac{\partial A}{\partial \mathcal{T}}\right) .
$$

In summary, for the distinguished scaling $M=\mathcal{O}\left(\epsilon^{1 / 4}\right)$, the system to determine the nonlinear evolution of the perturbation consists of the coupled equations (3.8), (3.18) and (3.21), subject to a suitable upstream condition to be prescribed in $\S 4.4$.

\section{Extensions to fully compressible regime}

\subsection{The case $T_{c}^{\prime}=0, M=\mathcal{O}(1)$}

We note that for the tanh profile (2.14), $T_{c}^{\prime}=0$ when $\beta_{T}=1$ even for $M=\mathcal{O}(1)$. It follows that the weakly nonlinear formulation of Goldstein \& Leib (1989) degenerates to a linear theory. In this section, we will show that the critical layer dynamics is strongly nonlinear as in the previous section, however the evolution equations must be slightly modified.

Suppose that $T_{c}^{\prime}=0$. Then the outer solution (2.30) shows that the temperature perturbation at leading order is

$$
\widetilde{T}_{0}=\left(T_{c}^{\prime \prime} / U_{c}^{\prime}+(\gamma-1) M^{2} U_{c}^{\prime}\right)\left(A \mathrm{e}^{\mathrm{i} \zeta}+\text { c.c. }\right)+\mathcal{O}(y),
$$

i.e. $\widetilde{T}_{0}$ is regular. However, the second-order solution (2.37) is singular, behaving as

$$
\widetilde{T}_{1}^{(1)}=\frac{\mathrm{i}}{\alpha} \frac{T_{c}^{\prime \prime}}{U_{c}^{\prime 2}}\left(A_{\mathcal{T}}+c A_{\mathcal{X}}\right) \frac{1}{y}+\mathcal{O}(\ln |y|) .
$$


Within the critical layer, the energy equation at leading order now reads (cf. (3.8))

$$
\mathcal{L}_{N} \bar{T}_{0}+Y T_{c}^{\prime \prime} \bar{v}_{0}=(\gamma-1) T_{c} M^{2}\left(\alpha U_{c}^{\prime} Y \bar{p}_{0 \zeta}+\bar{p}_{0 \mathcal{T}}+c \bar{p}_{0 \mathcal{X}}\right),
$$

where the solution for $\bar{v}_{0}$ and $\bar{p}_{0}$ is still given by (3.7). Thus after the substitution

$$
\widehat{T}=\bar{T}_{0}-\left(T_{c}^{\prime \prime} / U_{c}^{\prime}+(\gamma-1) M^{2} U_{c}^{\prime}\right)\left(A \mathrm{e}^{\mathrm{i} \zeta}+\text { c.c. }\right),
$$

we obtain

$$
\mathcal{L}_{N} \widehat{T}=-\frac{T_{c}^{\prime \prime}}{U_{c}^{\prime}}\left\{\left(A_{\mathcal{T}}+c A_{\mathcal{X}}\right) \mathrm{e}^{\mathrm{i} \zeta}+\text { c.c. }\right\} .
$$

Note that the right-hand side differs from that of (3.8), and that

$$
\widehat{T} \rightarrow \frac{\mathrm{i}}{\alpha} \frac{T_{c}^{\prime \prime}}{U_{c}^{\prime 2}}\left(A_{\mathcal{T}}+c A_{\mathcal{X}}\right) \frac{1}{Y} \mathrm{e}^{\mathrm{i} \zeta}+\text { c.c. } \quad \text { as } \quad Y \rightarrow \pm \infty
$$

to match with the outer solution (4.2).

On including finite Mach-number terms, equations (3.13)-(3.15) are modified to

$$
\begin{aligned}
& \gamma T_{c} M^{2}\left(\alpha U_{c}^{\prime} Y \bar{p}_{0 \zeta}+\bar{p}_{0 \mathcal{T}}+c \bar{p}_{0 \mathcal{X}}\right)-T_{c}^{\prime \prime} Y \bar{v}_{0} \\
& -\left(\alpha U_{c}^{\prime} Y \bar{T}_{0 \zeta}+\bar{T}_{0 \mathcal{T}}+c \bar{T}_{0 \mathcal{X}}+\bar{v}_{0} \bar{T}_{0 Y}\right)+T_{c}\left(\alpha \bar{u}_{1 \zeta}+\bar{u}_{0 \mathcal{X}}+\bar{v}_{2 Y}\right)=0, \\
& \mathcal{L}_{N} \bar{u}_{1}+Y U_{c}^{\prime} \bar{u}_{0 \mathcal{X}}+\alpha \bar{u}_{0} \bar{u}_{0 \zeta}+\gamma M^{2} U_{c}^{\prime} \bar{p}_{0} \bar{v}_{0}+U_{c}^{\prime} \bar{v}_{2}+\frac{1}{2} U_{c}^{\prime \prime \prime} Y^{2} \bar{v}_{0} \\
& =-\alpha T_{c} \bar{p}_{2 \zeta}-T_{c} \bar{p}_{1 \mathcal{X}}-\frac{1}{2} \alpha T_{c}^{\prime \prime} Y^{2} \bar{p}_{0 \zeta}-\alpha \bar{T}_{0} \bar{p}_{0 \zeta}+\Lambda T_{c} U_{c}^{\prime} \mu_{c}^{\prime} \bar{T}_{0 Y}, \\
& \alpha U_{c}^{\prime} Y \bar{v}_{0 \zeta}+\bar{v}_{0 \mathcal{T}}+c \bar{v}_{0 \mathcal{X}}=-T_{c} \bar{p}_{2 Y},
\end{aligned}
$$

where we have used the fact that $T_{c}^{\prime}=0$ and $U_{c}^{\prime \prime}=0$ (which follows from (2.25)). Following the same procedure as deriving (3.16), we find

$$
\begin{aligned}
\mathcal{L}_{N} \bar{u}_{1 Y}= & -\Lambda U_{c}^{\prime}\left(\mu_{c}-T_{c} \mu_{c}^{\prime}\right) \bar{T}_{0 Y Y}+\frac{U_{c}^{\prime}}{T_{c}} \bar{v}_{0} \bar{T}_{0 Y}+U_{c}^{\prime}\left(\frac{T_{c}^{\prime \prime}}{T_{c}}-\frac{U_{c}^{\prime \prime \prime}}{U_{c}^{\prime}}\right) Y \bar{v}_{0} \\
& +\alpha\left(\alpha U_{c}^{\prime} Y \bar{v}_{0 \zeta \zeta}+\bar{v}_{0 \mathcal{T} \zeta}+c \bar{v}_{0 \mathcal{X} \zeta}\right)+U_{c}^{\prime} M^{2}\left(\alpha U_{c}^{\prime} Y \bar{p}_{0 \zeta}+\bar{p}_{0 \mathcal{T}}+c \bar{p}_{0 \mathcal{X}}\right) .
\end{aligned}
$$

Substitution of

$$
\bar{u}_{1 Y}=\widehat{Q}+\left[\left(\alpha^{2}+U_{c}^{\prime \prime \prime} / U_{c}^{\prime}-T_{c}^{\prime \prime} / T_{c}+M^{2} U_{c}^{\prime 2} / T_{c}\right) A \mathrm{e}^{\mathrm{i} \zeta}+\text { c.c. }\right]
$$

into (4.9) reduces it to

$$
\mathcal{L}_{N} \widehat{Q}=\left(\frac{T_{c}^{\prime \prime}}{T_{c}}-\frac{U_{c}^{\prime \prime \prime}}{U_{c}^{\prime}}\right)\left\{\left(A_{\mathcal{T}}+c A_{\mathcal{X}}\right) \mathrm{e}^{\mathrm{i} \zeta}+\text { c.c. }\right\}-\frac{U_{c}^{\prime}}{T_{c}}\left(\mathrm{i} \alpha A \mathrm{e}^{\mathrm{i} \zeta}+\text { c.c. }\right) \widehat{T}_{Y}-\Lambda U_{c}^{\prime} \mu_{c} \sigma_{c} \widehat{T}_{Y Y} .
$$

The amplitude equation (3.21) remains intact. 

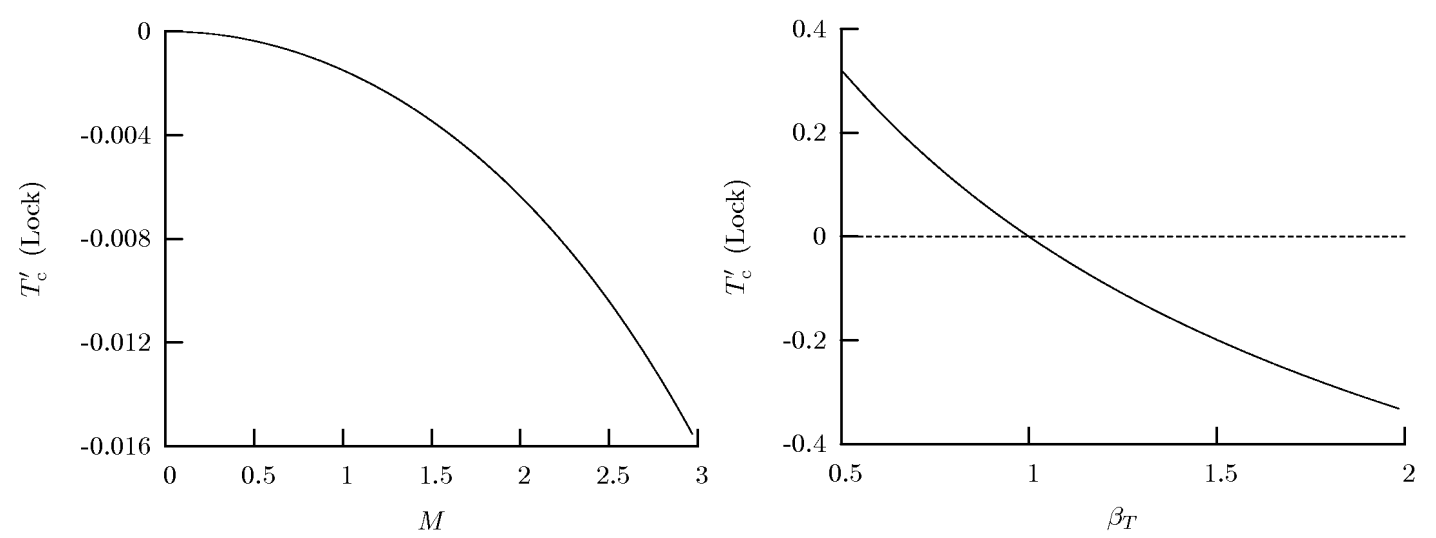

Figure 2: $T_{c}^{\prime}$ for the Lock profile with $\beta_{U}=1 / 2$. Left: $T_{c}^{\prime}$ vs. $M\left(\beta_{T}=1\right)$. Right: $T_{c}^{\prime}$ vs. $\beta_{T}$ $(M=1 / 2)$.

\subsection{Composite theory}

Numerical solutions of (2.11)-(2.12) (with $P_{r}=1$ ) indicate that for the Lock profile, $T_{c}^{\prime} \neq 0$ in general. Nevertheless for any $M$, there exists a value of $\beta_{T}$ for which $T_{c}^{\prime}$ equals zero (figure 2). Moreover, $T_{c}^{\prime}$ is numerically rather small for a range of order one $M$ and $\beta_{T}$ of interest. This implies that the weakly nonlinear theory based on the scaling $T_{c}^{\prime}=\mathcal{O}(1)$ is unlikely to be accurate or appropriate. To overcome this shortcoming, we tacitly treat $T_{c}^{\prime}$ as an independent parameter with $T_{c}^{\prime}=\mathcal{O}\left(\epsilon^{1 / 2}\right)$, whilst still treating $M=\mathcal{O}(1)$, so that a strongly nonlinear critical-layer theory may be formulated. The resulting system can be obtained by combining those given in $\S 3$ and in the previous subsection. We thus have

$$
\begin{gathered}
\mathcal{L}_{N} \widehat{T}=\mathrm{i} \alpha T_{c M}^{\prime} \mathcal{M}^{2}\left(A \mathrm{e}^{\mathrm{i} \zeta}-\text { c.c. }\right)-\frac{T_{c}^{\prime \prime}}{U_{c}^{\prime}}\left\{\left(A_{\mathcal{T}}+c A_{\mathcal{X}}\right) \mathrm{e}^{\mathrm{i} \zeta}+\text { c.c. }\right\} \\
\mathcal{L}_{N} \widehat{Q}=\left(\frac{T_{c}^{\prime \prime}}{T_{c}}-\frac{U_{c}^{\prime \prime \prime}}{U_{c}^{\prime}}\right)\left\{\left(A_{\mathcal{T}}+c A_{\mathcal{X}}\right) \mathrm{e}^{\mathrm{i} \zeta}+\text { c.c. }\right\}-\frac{U_{c}^{\prime}}{T_{c}}\left(\mathrm{i} \alpha A \mathrm{e}^{\mathrm{i} \zeta}+\text { c.c. }\right) \widehat{T}_{Y}-\Lambda U_{c}^{\prime} \mu_{c} \sigma_{c} \widehat{T}_{Y Y}, \\
\int_{-\infty}^{\infty} \int_{0}^{2 \pi} \widehat{Q} \mathrm{e}^{-\mathrm{i} \zeta} \mathrm{d} \zeta \mathrm{d} Y=4 \pi \mathrm{i} \frac{T_{c}}{\alpha^{2}}\left(c J_{1} \frac{\partial A}{\partial \mathcal{X}_{1}}+J_{2} \frac{\partial A}{\partial \mathcal{T}}\right)
\end{gathered}
$$

where the temperature equation (4.12) includes linear inhomogeneous terms involving both $T_{c}^{\prime}$ and $T_{c}^{\prime \prime}$, and the vorticity equation (4.13) includes linear inhomogeneous terms involving $U_{c}^{\prime \prime \prime}$ and $T_{c}^{\prime \prime}$. We note that

- For $M=0, T_{c}^{\prime}=T_{c}^{\prime \prime}=0$, and it follows that $\widehat{T} \equiv 0$. Then system (4.12)-(4.14) reduces to that of Goldstein \& Hultgren (1988), as expected.

- For $M=\mathcal{O}\left(\epsilon^{1 / 4}\right)$, system (4.12)-(4.14) reduces to (3.8), (3.18) and (3.21).

- If $T_{c}^{\prime}=0$ and $M=\mathcal{O}(1)$, system (4.12)-(4.14) simplifies to (4.5) and (4.11).

Furthermore, we shall show that in the limit $T_{c M}^{\prime} \mathcal{M}^{2} \gg 1$ (due to either $\mathcal{M} \gg 1$ or $|\hat{\beta}| \gg 1$ ), one may derive the amplitude equation in the weakly nonlinear theory directly from (4.12)-(4.14), which implies that the former is "contained" in the latter. Therefore, the strongly nonlinear system (4.12)-(4.14) represents a composite approximation, valid for $M=0$ to $M=\mathcal{O}(1)$ for an arbitrary $\beta_{T}$. 


\subsection{Decomposition of the solution}

The system (4.12)-(4.14) is derived for a modulated wavetrain. In the following, it will be applied to a simpler case where a single mode is excited upstream. The amplitude $A$ takes on the form

$$
A(\mathcal{X}, \mathcal{T})=\mathrm{e}^{\mathrm{i} \Omega \mathcal{T}} \widehat{A}(\mathcal{X})
$$

and $\Omega=\left(\omega_{n}-\omega\right) / \epsilon^{1 / 2}$ represents a (scaled) deviation of the forcing frequency from the neutral frequency. The ensuing disturbance initially consists primarily of a fundamental wave only, and so the appropriate upstream condition can be prescribed as

$$
\widehat{A}(\mathcal{X}) \rightarrow a \mathrm{e}^{\kappa \mathcal{X}} \quad \text { as } \quad \mathcal{X} \rightarrow-\infty
$$

where $\Re\{\kappa\}$ is the linear growth rate, and $a$ is a (complex) amplitude parameter.

To remove the parameters $\Omega$ and $a$ from (4.12)-(4.14) and (4.16), we now introduce the re-scaled variables and parameters (cf. Goldstein \& Hultgren 1988),

$$
\left.\begin{array}{c}
\overline{\mathcal{X}}=(\alpha \Omega / 2) \mathcal{X}-x_{0}, \quad \bar{Y}=(2 / \alpha c \Omega)\left(\alpha U_{c}^{\prime} Y+\Omega\right), \quad \bar{\zeta}=\zeta+\arg a+\bar{\kappa}_{\mathrm{i}} x_{0}, \\
\bar{A}=\left(8 U_{c}^{\prime} / c^{2} \Omega^{2}\right) \widehat{A}, \quad(\bar{Q}, \bar{T})=-4\left(\alpha U_{c}^{\prime 2} / c \Omega^{2} U_{c}^{\prime \prime \prime}\right)(\widehat{Q}, \widehat{T}), \\
\overline{\mathcal{M}}^{2}=-\left(\alpha U_{c}^{\prime 2}\left|T_{c \mathrm{M}}^{\prime}\right| / \Omega T_{c} U_{c}^{\prime \prime \prime}\right) \mathcal{M}^{2}, \quad \bar{\Lambda}=8 T_{c} \mu_{c}\left(U_{c}^{\prime 2} / \alpha c^{3} \Omega^{3}\right) \Lambda, \quad \bar{\kappa}=(2 / \alpha \Omega) \kappa,
\end{array}\right\}
$$

where $\bar{\kappa}=\bar{\kappa}_{\mathrm{r}}+\mathrm{i} \bar{\kappa}_{\mathrm{i}}$, and $x_{0}$ is chosen to be $x_{0}=\left(1 / \bar{\kappa}_{\mathrm{r}}\right) \ln \left[c^{2} \Omega^{2} /\left(8 U_{c}^{\prime}|a|\right)\right]$.

As the disturbance evolves into the nonlinear regime, all harmonics are simultaneously generated at the same order, and so $\bar{T}$ and $\bar{Q}$ take the form

$$
(\bar{Q}, \bar{T})=\sum_{m=-\infty}^{\infty}\left(\bar{Q}_{m}(\overline{\mathcal{X}}, \bar{Y}), \bar{T}_{m}(\overline{\mathcal{X}}, \bar{Y})\right) \mathrm{e}^{\mathrm{i} m(\bar{\zeta}+\Omega \mathcal{T})},
$$

Substituting (4.17) and (4.18) into (4.12)-(4.14), we obtain

$$
\begin{aligned}
&\left(\frac{\partial}{\partial \overline{\mathcal{X}}}+\mathrm{i} m \bar{Y}\right) \bar{T}_{m}+ \frac{\mathrm{i}}{2} \frac{\partial}{\partial \bar{Y}}\left(\bar{A}^{*} \bar{T}_{m+1}-\bar{A} \bar{T}_{m-1}\right)-\bar{\Lambda} \frac{\partial^{2} \bar{T}_{m}}{\partial \bar{Y}^{2}}=\delta_{m, 1}\left[\mathrm{i} \overline{\mathcal{M}}^{2} \bar{A}-\sigma\left(\frac{\alpha c}{2} \frac{\mathrm{d}}{\mathrm{d} \overline{\mathcal{X}}}+\mathrm{i}\right) \bar{A}\right] \\
&\left(\frac{\partial}{\partial \overline{\mathcal{X}}}+\mathrm{i} m \bar{Y}\right) \bar{Q}_{m}+\frac{\mathrm{i}}{2} \frac{\partial}{\partial \bar{Y}}\left[\bar{A}^{*}\left(\bar{Q}_{m+1}-\bar{T}_{m+1}\right)-\bar{A}\left(\bar{Q}_{m-1}-\bar{T}_{m-1}\right)\right] \\
&+\bar{\Lambda} \frac{\partial^{2}}{\partial \bar{Y}^{2}}\left(\sigma_{c} \bar{T}_{m}-\bar{Q}_{m}\right)=\delta_{m, 1}(1+\sigma)\left(\frac{\alpha c}{2} \frac{\mathrm{d} \bar{A}}{\mathrm{~d} \overline{\mathcal{X}}}+\mathrm{i} \bar{A}\right) \\
& \int_{-\infty}^{\infty} \bar{Q}_{1}(\overline{\mathcal{X}}, \bar{Y}) \mathrm{d} \bar{Y}=-2 \mathrm{i} \frac{T_{c} U_{c}^{\prime 2}}{\alpha U_{c}^{\prime \prime \prime}}\left(\frac{\alpha c}{2} J_{1} \frac{\mathrm{d}}{\mathrm{d} \overline{\mathcal{X}}}+\mathrm{i} J_{2}\right) \bar{A}
\end{aligned}
$$

where

$$
\sigma=-U_{c}^{\prime} T_{c}^{\prime \prime} /\left(T_{c} U_{c}^{\prime \prime \prime}\right) .
$$

The initial condition (4.16) becomes

$$
\bar{A}(\overline{\mathcal{X}}) \rightarrow \mathrm{e}^{\bar{\kappa} \overline{\mathcal{X}}} \quad \text { as } \quad \overline{\mathcal{X}} \rightarrow-\infty .
$$




\subsection{Initial condition and linear critical layer upstream}

To determine the appropriate initial condition for the vorticity and temperature, we note that the flow upstream is governed by linear dynamics so that

$$
\bar{T}_{1}(\overline{\mathcal{X}}, \bar{Y}) \rightarrow \bar{T}_{L}(\bar{Y}) \mathrm{e}^{\bar{\kappa} \overline{\mathcal{X}}}, \quad \bar{Q}_{1}(\overline{\mathcal{X}}, \bar{Y}) \rightarrow \bar{Q}_{L}(\bar{Y}) \mathrm{e}^{\bar{\kappa} \overline{\mathcal{X}}} \quad \text { as } \quad \overline{\mathcal{X}} \rightarrow-\infty .
$$

Functions $\bar{T}_{L}$ and $\bar{Q}_{L}$ satisfy (4.19) and (4.20) with the nonlinear terms dropped. They are solved by taking Fourier transform with respect to $\bar{Y}$ to give

$$
\begin{aligned}
& \bar{T}_{L}(\bar{Y})=\left(\mathrm{i} \overline{\mathcal{M}}^{2}-\sigma\left(\frac{\alpha c}{2} \bar{\kappa}+\mathrm{i}\right)\right) \int_{0}^{\infty} \mathrm{e}^{-\left(\frac{\bar{\Lambda}}{3} \lambda^{3}+\bar{\kappa} \lambda+\mathrm{i} \bar{Y} \lambda\right)} \mathrm{d} \lambda, \\
& \bar{Q}_{L}(\bar{Y})=(1+\sigma)\left(\frac{\alpha c}{2} \bar{\kappa}+\mathrm{i}\right) \int_{0}^{\infty} \mathrm{e}^{-\left(\frac{\bar{\Lambda}}{3} \lambda^{3}+\bar{\kappa} \lambda+\mathrm{i} \bar{Y} \lambda\right)} \mathrm{d} \lambda \\
&+\frac{1}{3} \bar{\Lambda} \sigma_{c}\left(\mathrm{i} \overline{\mathcal{M}}^{2}-\sigma\left(\frac{\alpha c}{2} \bar{\kappa}+\mathrm{i}\right)\right) \int_{0}^{\infty} \lambda^{3} \mathrm{e}^{-\left(\frac{\bar{\Lambda}}{3} \lambda^{3}+\bar{\kappa} \lambda+\mathrm{i} \bar{Y} \lambda\right)} \mathrm{d} \lambda,
\end{aligned}
$$

Substitution of (4.23) and (4.26) into (4.21) yields

$$
\bar{\kappa}=\frac{2}{\alpha c}\left[\frac{2\left(J_{2}-J_{1}\right) T_{c} U_{c}^{\prime 2}}{\pi \alpha U_{c}^{\prime \prime \prime}(1+\sigma)+2 \mathrm{i} T_{c} U_{c}^{\prime 2} J_{1}}-\mathrm{i}\right] .
$$

\subsection{Weakly nonlinear limit: amplitude equation}

In this section, we shall show that for $\overline{\mathcal{M}} \gg 1$, the weakly nonlinear theory of Golsdtein \& Leib (1989) can be recovered from system (4.19)-(4.21). We perform the substitutions

$$
\overline{\mathcal{X}}=\widetilde{\mathcal{X}}+\bar{x}_{0}, \quad \bar{A}=\overline{\mathcal{M}}^{-1} \widetilde{A} \mathrm{e}^{\mathrm{i} \bar{\kappa}_{i} \bar{x}_{0}}, \quad\left(\bar{T}_{m}, \bar{Q}_{m}\right)=\overline{\mathcal{M}}\left(\widetilde{T}_{m}, \widetilde{Q}_{m}\right) \mathrm{e}^{m \mathrm{i} \bar{\kappa}_{i} \bar{x}_{0}},
$$

where $\bar{x}_{0}=-\left(1 / \bar{\kappa}_{r}\right) \ln \overline{\mathcal{M}}$, and expand $\bar{T}_{m}$ and $\bar{Q}_{m}$ as

$$
\left(\widetilde{T}_{m}, \widetilde{Q}_{m}\right)=\left(\widetilde{T}_{m}^{(0)}, \widetilde{Q}_{m}^{(0)}\right)+\overline{\mathcal{M}}^{-1}\left(\widetilde{T}_{m}^{(1)}, \widetilde{Q}_{m}^{(1)}\right)+\overline{\mathcal{M}}^{-2}\left(\widetilde{T}_{m}^{(2)}, \widetilde{Q}_{m}^{(2)}\right)+\cdots
$$

with $\widetilde{T}_{m}^{(0)}=\widetilde{Q}_{m}^{(0)}=0$ for $m \neq-1,1$. Inserting (4.28)-(4.29) into (4.19)-(4.21) leads to a series of equations for $\widetilde{T}_{m}^{(j)}$ and $\widetilde{Q}_{m}^{(j)}(j=0,1,2)$. These equations are given in (A.1)-(A.8) in the appendix, where they are solved in sequence to give the amplitude equation,

$$
\frac{\mathrm{d} \widetilde{A}}{\mathrm{~d} \widetilde{\mathcal{X}}}=\tilde{\kappa} \widetilde{A}+\mathcal{C} \int_{0}^{\infty} \int_{0}^{\infty} \aleph(\lambda, \mu ; \bar{\Lambda}) \widetilde{A}(\widetilde{\mathcal{X}}-\lambda) \widetilde{A}(\widetilde{\mathcal{X}}-\lambda-\mu) \widetilde{A}^{*}(\widetilde{\mathcal{X}}-2 \lambda-\mu) \mathrm{d} \mu \mathrm{d} \lambda,
$$

where

$$
\begin{gathered}
\mathcal{C}=\frac{\pi \alpha U_{c}^{\prime \prime \prime} \operatorname{sgn}\left(T_{c}^{\prime}\right) \tilde{\kappa}}{\pi \alpha U_{c}^{\prime \prime \prime}(1+\sigma)+2 \mathrm{i} T_{c} U_{c}^{\prime 2} J_{2}}, \\
\aleph(\lambda, \mu ; \bar{\Lambda})=\lambda^{2}\left[1-\frac{1}{6} \bar{\Lambda}\left(1-T_{c} \mu_{c}^{\prime} / \mu_{c}\right) \lambda^{2}(2 \lambda+3 \mu)\right] \mathrm{e}^{-\frac{1}{3} \bar{\Lambda}(2 \lambda+3 \mu) \lambda^{2}} .
\end{gathered}
$$

The initial condition (4.23) becomes

$$
\widetilde{A}(\tilde{\mathcal{X}}) \rightarrow \mathrm{e}^{\tilde{\kappa} \tilde{\mathcal{X}}} \quad \text { as } \quad \tilde{\mathcal{X}} \rightarrow-\infty .
$$




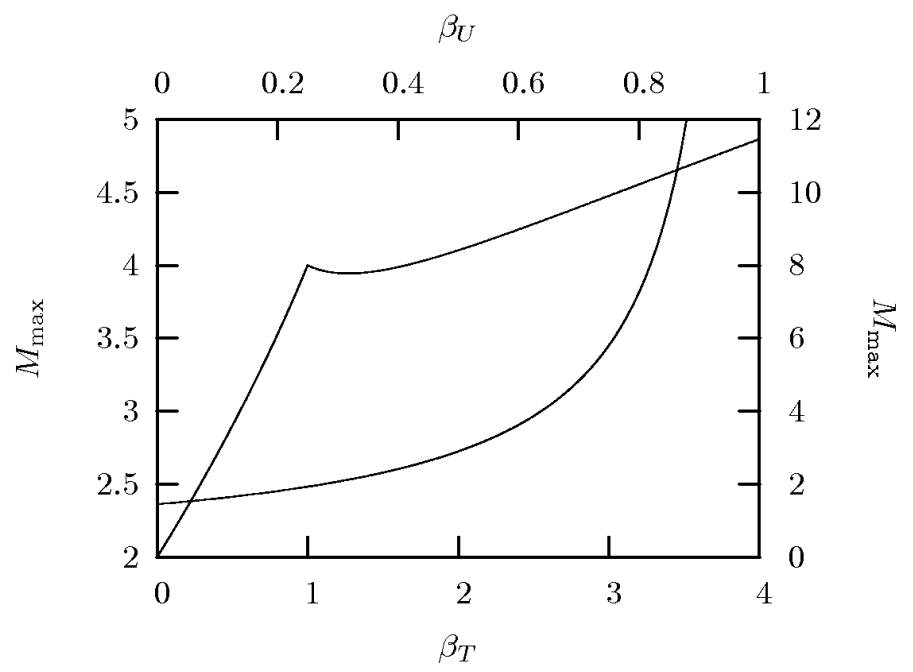

Figure 3: Maximum Mach numbers for the existence of subsonic modes. Top graph: variation with $\beta_{T}$ for $\beta_{U}=1 / 2$. Bottom graph: variation with $\beta_{U}$ for $\beta_{T}=1 / 2$.

This furnishes the demonstration that the weakly nonlinear theory of Goldstein \& Leib (1989) for $M=\mathcal{O}(1)$ can be recovered as a limiting case of the current strongly nonlinear formulation. Hence we can conclude that the latter theory is valid for all $0 \leqslant M \leqslant \mathcal{O}(1)$.

It may be pointed out that the present derivation of amplitude equation (4.30) was carried out entirely in the Fourier space without inverting any of the solutions to physical space; it is therefore more compact than that of Goldstein \& Leib (1989) and Leib (1991).

\section{$5 \quad$ Numerical solutions}

\subsection{Solution of eigenvalue problem and evaluation of the coefficients}

For most calculations, we will use the tanh velocity profile (2.14). It follows from (2.25) that $s$ in (2.14) is a root of the cubic equation

$$
(\gamma-1) M^{2}\left(\beta_{U}-1\right)^{2} \tanh ^{3}(s)+\left[4\left(1+\beta_{T}\right)-(\gamma-1) M^{2}\left(\beta_{U}-1\right)^{2}\right] \tanh (s)+4\left(1-\beta_{T}\right)=0 .
$$

In all the calculations to be presented in this paper, we take $\gamma=7 / 5$, which is close to the ratio for air $\left(\approx 1.401\right.$ at $1 \mathrm{~atm}$. and $\left.15^{\circ} \mathrm{C}\right)$. The use of the tanh profile $(2.14)$ as an approximation to the mean velocity has been found only to produce quantitative differences in linear instability properties (Jackson \& Grosch 1991), and in weakly nonlinear evolution (Leib 1991) provided that $\left(\beta_{T}-1\right)=\mathcal{O}(1)$. The quantitative features of strongly nonlinear evolution are representative of other profiles, as is shown in Sparks (2006).

After finding $s$ from (5.1), the neutral phase speed $c$ is given by

$$
c=\bar{U}(0)=\frac{1}{2}\left(1+\beta_{U}+\left(1-\beta_{U}\right) \tanh (s)\right) .
$$

Rayleigh equation (2.23), rewritten in terms of Howarth-Dorodnitsyn variable $\eta$, is then solved by a shooting method to determine the eigenvalue $\alpha$. Eigenvalues and eigenfunctions for representative parameter values are given in Sparks (2006). As was found previously, there exists a maximum Mach number, $M_{\max }$, beyond which subsonic modes no longer exist. Some plots of $M_{\max }$ against $\beta_{U}$ and $\beta_{T}$ are shown in figure 3. Subsonic and 
supersonic modes may co-exist for a small range of Mach numbers close to $M_{\max }$ (as shown for example in figure 7(a) of Jackson \& Grosch (1989)).

The integrals such as $J_{2}$ and $J_{3}$ in (2.39) are approximated over a large but finite domain $(-H, H)$, which is further sub-divided into $(-H,-\delta),(-\delta, \delta)$ and $(\delta, H)$. Over $(-\delta, \delta)$, the integrands are singular but can be approximated by their asymptotic expressions so that the Hadamard finite part of the integrals can be found analytically. The integrals over $(-H,-\delta)$ and $(\delta, H)$ are evaluated numerically using Simpson's rule.

\subsection{Nonlinear calculation: strongly nonlinear evolution system}

Solving the nonlinear evolution system (4.19)-(4.21) numerically requires the boundary conditions on $\bar{T}_{m}$ and $\bar{Q}_{m}$ for large $\bar{Y}$. These can be derived by considering the dominant balance for $|\bar{Y}| \gg 1$. In (4.19), balancing i $m \bar{Y} \bar{T}_{1}$ with the right-hand side shows that

$$
\bar{T}_{1} \sim\left\{\sigma\left(\mathrm{i} \frac{\alpha c}{2} \frac{\mathrm{d} \bar{A}}{\mathrm{~d} \overline{\mathcal{X}}}-\bar{A}\right)+\overline{\mathcal{M}}^{2} \bar{A}\right\} \frac{1}{\bar{Y}}+\left\{\mathrm{i} \overline{\mathcal{M}}^{2} \frac{\mathrm{d} \bar{A}}{\mathrm{~d} \overline{\mathcal{X}}}-\sigma\left(\frac{\alpha c}{2} \frac{\mathrm{d}^{2} \bar{A}}{\mathrm{~d} \overline{\mathcal{X}}^{2}}+\mathrm{i} \frac{\mathrm{d} \bar{A}}{\mathrm{~d} \overline{\mathcal{X}}}\right)\right\} \frac{1}{\bar{Y}^{2}}+\mathcal{O}\left(\frac{1}{\bar{Y}^{3}}\right) .
$$

Next, balancing $\partial \bar{T}_{0} / \partial \overline{\mathcal{X}}$ with $\frac{\mathrm{i}}{2}(\partial / \partial \bar{Y})\left(\bar{A}^{*} \bar{T}_{1}-\bar{A} \bar{T}_{-1}\right)$ demonstrates that

$$
\bar{T}_{0} \sim-\frac{1}{4} \alpha c \sigma|\bar{A}|^{2} \frac{1}{\bar{Y}^{2}}-\left\{\overline{\mathcal{M}}^{2}|\bar{A}|^{2}-\sigma\left[|A|^{2}-\frac{\mathrm{i} \alpha c}{2}\left(\bar{A}^{*} \bar{A}^{\prime}-\bar{A} \bar{A}^{\prime *}\right)\right]\right\} \frac{1}{\bar{Y}^{3}}+\mathcal{O}\left(\frac{1}{\bar{Y}^{4}}\right) .
$$

Balancing $2 \mathrm{i} \bar{Y} \bar{T}_{2}$ with $-\frac{\mathrm{i}}{2} \bar{A}\left(\partial \bar{T}_{1} / \partial \bar{Y}\right)$ shows that

$$
\bar{T}_{2} \sim-\frac{1}{4}\left\{\sigma \bar{A}\left(\mathrm{i} \frac{\alpha c}{2} \frac{\mathrm{d} \bar{A}}{\mathrm{~d} \overline{\mathcal{X}}}-\bar{A}\right)+\overline{\mathcal{M}}^{2} \bar{A}^{2}\right\} \frac{1}{\bar{Y}^{3}}+\mathcal{O}\left(\frac{1}{\bar{Y}^{4}}\right)
$$

Similarly from equation (4.20), we find that as $\bar{Y} \rightarrow \pm \infty$,

$$
\begin{aligned}
& \bar{Q}_{0} \sim \frac{1}{4} \alpha c(1+2 \sigma)|\bar{A}|^{2} \frac{1}{\overline{Y^{2}}}+\mathcal{O}\left(\frac{1}{\bar{Y}^{3}}\right), \\
& \bar{Q}_{1} \sim(1+\sigma)\left(\bar{A}-\mathrm{i} \frac{\alpha c}{2} \frac{\mathrm{d} \bar{A}}{\mathrm{~d} \overline{\mathcal{X}}}\right) \frac{1}{\bar{Y}}+(1+\sigma)\left(\frac{\alpha c}{2} \frac{\mathrm{d}^{2} \bar{A}}{\mathrm{~d} \overline{\mathcal{X}}^{2}}+\mathrm{i} \frac{\mathrm{d} \bar{A}}{\mathrm{~d} \overline{\mathcal{X}}}\right) \frac{1}{\bar{Y}^{2}}+\mathcal{O}\left(\frac{1}{\bar{Y}^{3}}\right), \\
& \bar{Q}_{2} \sim \frac{1}{4}\left\{(1+2 \sigma) \bar{A}\left(\mathrm{i} \frac{\alpha c}{2} \frac{\mathrm{d} \bar{A}}{\mathrm{~d} \overline{\mathcal{X}}}-\bar{A}\right)+\overline{\mathcal{M}}^{2} \bar{A}^{2}\right\} \frac{1}{\bar{Y}^{3}}+\mathcal{O}\left(\frac{1}{\bar{Y}^{4}}\right) .
\end{aligned}
$$

Generally for $\mathrm{m} \geqslant 3$,

$$
\bar{T}_{m} \sim \mathcal{O}\left(\bar{Y}^{-(2 m-1)}\right), \quad \bar{Q}_{m} \sim \mathcal{O}\left(\bar{Y}^{-(2 m-1)}\right) \quad \text { as } \quad \bar{Y} \rightarrow \pm \infty .
$$

On truncating the integral over the domain $[-H, H]$, we can write $(4.21)$ as

$$
-2 \mathrm{i} \frac{T_{c} U_{c}^{\prime 2}}{\alpha U_{c}^{\prime \prime \prime}}\left(\frac{\alpha c}{2} J_{1} \frac{\mathrm{d}}{\mathrm{d} \overline{\mathcal{X}}}+\mathrm{i} J_{2}\right) \bar{A}=I_{1}+2 \frac{(1+\sigma)}{H}\left(\frac{\alpha c}{2} \frac{\mathrm{d}^{2} \bar{A}}{\mathrm{~d} \overline{\mathcal{X}}^{2}}+\mathrm{i} \frac{\mathrm{d} \bar{A}}{\mathrm{~d} \overline{\mathcal{X}}}\right)+\mathcal{O}\left(H^{-3}\right),
$$

where we have defined

$$
I_{n}=\int_{-H}^{H} \bar{Y}^{n} \bar{Q}_{1} \mathrm{~d} \bar{Y}
$$

Now differentiate (5.10) (with the $\mathcal{O}(1 / H)$ term dropped) with respect to $\overline{\mathcal{X}}$ and use $(4.20)$ for $\partial \bar{Q}_{1} / \partial \overline{\mathcal{X}}$. The resulting equation is then combined with (5.10) to eliminate $\mathrm{d}^{2} \bar{A} / \mathrm{d} \overline{\mathcal{X}}^{2}$, 
leading to

$$
\begin{aligned}
& \left\{c\left(\frac{U_{c}^{\prime 2} T_{c}^{2}}{U_{c}^{\prime \prime \prime}} J_{1}^{2}+\alpha^{2}(1+\sigma)^{2} \frac{U_{c}^{\prime \prime \prime}}{U_{c}^{\prime 2}}\right)+2 T_{c}\left(J_{1}-J_{2}\right) \frac{(1+\sigma)}{H}\right\} \frac{\mathrm{d} \bar{A}}{\mathrm{~d} \overline{\mathcal{X}}} \\
& +\frac{2 \mathrm{i}}{\alpha}\left\{\frac{U_{c}^{\prime 2} T_{c}^{2}}{U_{c}^{\prime \prime \prime}} J_{1} J_{2}+\alpha^{2}(1+\sigma)^{2} \frac{U_{c}^{\prime \prime \prime}}{U_{c}^{\prime 2}}\right\} \bar{A}=\mathrm{i} T_{c} J_{1} I_{0}+\mathrm{i} \alpha \frac{(1+\sigma)}{H} \frac{U_{c}^{\prime \prime \prime}}{U_{c}^{\prime 2}} I_{1}+\mathcal{O}\left(H^{-2}\right) .
\end{aligned}
$$

The nonlinear evolution system is solved numerically by using a predictor-corrector method. In the predicting step, (5.11) is discretised using a fourth-order Adams-Bashforth scheme, whilst (4.19)-(4.20) are treated in a mixed 'implicit-explicit' fashion, namely a third-order implicit (Adams-Moulton) scheme for the linear terms, and an explicit (AdamsBashforth) scheme for the nonlinear terms. A central difference is used to approximate the derivatives with respect to $\bar{Y}$. The integrals are evaluated by Simpson's rule. The corrector consists of a fourth-order Adams-Moulton scheme for (5.11) and a third-order Adams-Moulton scheme for (4.19)-(4.20). The overall error of the method is of $\mathcal{O}\left(l^{3}, h^{2}\right)$, where $l$ and $h$ are the mesh sizes in the streamwise and transverse directions respectively.

The marching begins with the linear solution (4.23)-(4.24) at a sufficiently large but negative $\overline{\mathcal{X}}$. A value for $\bar{A}$ at the next step is predicted. The values for $\bar{T}$ and $\bar{Q}$ at the boundaries $\bar{Y}= \pm H$ are then computed using (5.3)-(5.9), the derivatives in which, $\mathrm{d} \bar{A} / \mathrm{d} \overline{\mathcal{X}}$ and $\mathrm{d}^{2} \bar{A} / \mathrm{d} \overline{\mathcal{X}}^{2}$, are approximated by fourth-order backward difference formulae. Then, $\bar{T}$ and $\bar{Q}$ at the next 'time' step are predicted by solving in sequence two trio-diagonal linear simultaneous systems. The correcting step is executed in a similar fashion. Further details of the numerical treatment and code validations are described in Sparks (2006).

The amplitude equation (4.30) was solved numerically by using a fourth-order AdamsBashforth scheme to advance the solution downstream from the upstream state (4.33).

\subsection{Vorticity and temperature within the critical layer}

The nonlinear dynamics of the critical layer will be illustrated by suitable contours of vorticity and temperature. The total vorticity within the critical layer is

$$
\Omega_{T}=-\frac{1}{\epsilon^{1 / 2}} \frac{\partial u}{\partial Y}+\left(\alpha \frac{\partial v}{\partial \zeta}+\epsilon^{1 / 2} \frac{\partial v}{\partial \mathcal{X}}+\Lambda \epsilon^{3 / 2} \frac{\partial v}{\partial \mathcal{X}_{2}}\right) \equiv-\left(\Omega_{0}+\epsilon^{1 / 2} \Omega_{1 / 2}+\epsilon \Omega_{1}+\cdots\right) .
$$

It follows from (2.8), (2.15), (3.4), (3.7), (3.12) and (4.10) that $\Omega_{0}=U_{c}^{\prime}, \Omega_{1 / 2}=0$, and

$$
\Omega_{1}=\frac{1}{2} U_{c}^{\prime \prime \prime} Y^{2}+\left(U_{c}^{\prime} T_{c M}^{\prime} \mathcal{M}^{2} / T_{c}\right) Y+\widehat{Q}+2\left[U_{c}^{\prime \prime \prime} / U_{c}^{\prime}-T_{c}^{\prime \prime} / T_{c}+M^{2} U_{c}^{\prime 2} / T_{c}\right] \Re\left\{A \mathrm{e}^{\mathrm{i} \zeta}\right\} .
$$

In the subsequent figures, we will plot the contours of the normalised $\mathcal{O}(\epsilon)$ vorticity

$$
\begin{aligned}
\bar{\Omega}_{1} & =\frac{8 U_{c}^{\prime 2}}{c^{2} U_{c}^{\prime \prime \prime} \Omega^{2}}\left[\Omega_{1}+\left(\frac{U_{c}^{\prime} T_{c M}^{\prime} \mathcal{M}^{2}}{U_{c}^{\prime \prime \prime} T_{c}}\right)^{2}-2 \frac{U_{c}^{\prime 2}}{T_{c}} M^{2} \Re\left\{A \mathrm{e}^{\mathrm{i} \zeta}\right\}\right] \\
& =\left[\bar{Y}-\frac{2}{\alpha c}\left(1+\overline{\mathcal{M}}^{2}\right)\right]^{2}+2(1+\sigma) \Re\left\{\bar{A} \mathrm{e}^{\mathrm{i} \tilde{\zeta}}\right\}-\frac{2}{\alpha c} \sum_{m=-\infty}^{\infty} \bar{Q}_{m} \mathrm{e}^{\mathrm{i} m \bar{\zeta}},
\end{aligned}
$$

where $\tilde{\zeta}=\zeta+\Omega \mathcal{T}$, and use has been made of equations (4.17).

Similarly, the total temperature within the critical layer is

$$
T=\tau_{0}+\epsilon^{1 / 2} \tau_{1 / 2}+\epsilon \tau_{1}+\cdots .
$$


Use of (2.8), (2.15), (3.4) and (4.4) shows that $\tau_{0}=T_{c}, \tau_{1 / 2}=0$ and

$$
\tau_{1}=T_{c M}^{\prime} \mathcal{M}^{2} Y+\frac{1}{2} T_{c}^{\prime \prime} Y^{2}+\widehat{T}+2\left(T_{c}^{\prime \prime} / U_{c}^{\prime}+(\gamma-1) M^{2} U_{c}^{\prime}\right) \Re\left\{A \mathrm{e}^{\mathrm{i} \zeta}\right\} .
$$

Temperature contours will be plotted in terms of the normalised $\mathcal{O}(\epsilon)$ temperature

$$
\begin{aligned}
\bar{\tau}_{1} & =-\frac{8 U_{c}^{\prime 3}}{c^{2} T_{c} U_{c}^{\prime \prime \prime} \Omega^{2}}\left[\tau_{1}+\left(\frac{T_{c M}^{\prime} \mathcal{M}^{2}}{T_{c}^{\prime \prime}}\right)^{2}-2(\gamma-1) M^{2} U_{c}^{\prime} \Re\left\{A \mathrm{e}^{\mathrm{i} \zeta}\right\}\right] \\
& =\sigma\left[\bar{Y}-\frac{2}{\alpha c}\left(1-\frac{\overline{\mathcal{M}}^{2}}{\sigma}\right)\right]^{2}+2 \sigma \Re\left\{\bar{A} \mathrm{e}^{\mathrm{i} \tilde{\zeta}}\right\}+\frac{2}{\alpha c} \sum_{m=-\infty}^{\infty} \bar{T}_{m} \mathrm{e}^{\mathrm{i} m \bar{\zeta}} .
\end{aligned}
$$

\subsection{Results}

Let us define

$$
\widehat{\Lambda}=\alpha c^{3} /\left(8 T_{c} \mu_{c} U_{c}^{\prime 2}\right) \bar{\Lambda}=\Lambda / \Omega^{3}=1 /\left(\hat{\omega}^{3} R\right),
$$

where $\hat{\omega}=\left(\omega_{n}-\omega\right) \ll 1$. This definition is convenient since $\widehat{\Lambda}$ is directly related to the important quantities $\hat{\omega}$ (the deviation from the neutral frequency) and $R$ (the Reynolds number). Also note that

$$
\overline{\mathcal{M}}^{2}=T_{c M}^{\prime} \mathcal{M}^{2} / \Omega=T_{c}^{\prime} / \hat{\omega}
$$

and so $\overline{\mathcal{M}}^{2}$ is proportional to the important physical quantity $T_{c}^{\prime}$ (controlled by Mach number $M$, and the velocity and temperature ratios, $\beta_{U}$ and $\beta_{T}$ ).

For the tanh profile, when $\beta_{T}=1, T_{c}^{\prime}=0$ and hence $\overline{\mathcal{M}}^{2}=0$ for all $M$. When $\beta_{T} \neq 1$, then $T_{c}^{\prime} \neq 0$ and $\overline{\mathcal{M}} \neq 0$. If we fix $\gamma, \beta_{U}$ and $M$, but decrease $\hat{\omega}$, then $\left|\overline{\mathcal{M}}^{2}\right|$ increases. The parameter $\left|\overline{\mathcal{M}}^{2}\right|$ may also be increased by increasing $\left|\beta_{T}-1\right|$ or $M$. These relations are worth noting since the validity of the weakly nonlinear theory, formally derived for $\left|\overline{\mathcal{M}}^{2}\right| \gg 1$, can be better related to physical parameters.

The calculations will be performed for the Chapman viscosity law $\mu=T$ and the tanh profile. The velocity ratio is taken to be a representative value $\beta_{U}=1 / 2$. Finally, we fix $\hat{\omega}=1 / 40$. The remaining three parameters are $M, \beta_{T}$ and $\hat{\Lambda}$. The present choice of $\hat{\omega}=1 / 40$ is based on two considerations. Firstly, in order for the mode to be nearlyneutral, $\hat{\omega}$ should not be much larger than this value. Secondly, we wish to focus on order-one $\overline{\mathcal{M}}$, which requires $\hat{\omega}$ not to be too small; for example, if $\beta_{U}=1 / 2, \beta_{T}=1 / 2$ and $M=1 / 2, \hat{\omega}=1 / 40$ gives $\overline{\mathcal{M}}^{2} \approx 2.33$, whilst if $\hat{\omega}=1 / 4000$ then $\overline{\mathcal{M}}^{2} \approx 233$. Primarily due to the choice of $\hat{\omega}$, the results to be presented in this section correspond to moderate $\overline{\mathcal{M}}$, for which the strongly nonlinear theory is required whilst the weakly nonlinear theory is not expected to be a good approximation. Parameters for which the weakly nonlinear theory is valid will be considered later in $\S 6.7$.

\subsection{Case I: $\beta_{T}=1$}

The first case corresponds to two streams of equal temperature $\left(\beta_{T}=1\right)$ and a relatively low Mach number $(M=1 / 2)$. Figure 4 shows the amplitude development and instantaneous growth rate for a range of $\widehat{\Lambda}$. For small $\widehat{\Lambda}$, the amplitude exhibits a sinusoidal-like behaviour with an oscillating growth rate. Increasing $\widehat{\Lambda}$ suppresses and eventually eliminates the oscillation, accompanied by an increase in the amplitude. For all $\widehat{\Lambda} \neq 0$, the amplitude eventually grows at a rate that ultimately tends to zero, similar to the incompressible case (cf. Goldstein \& Hultgren 1988). 

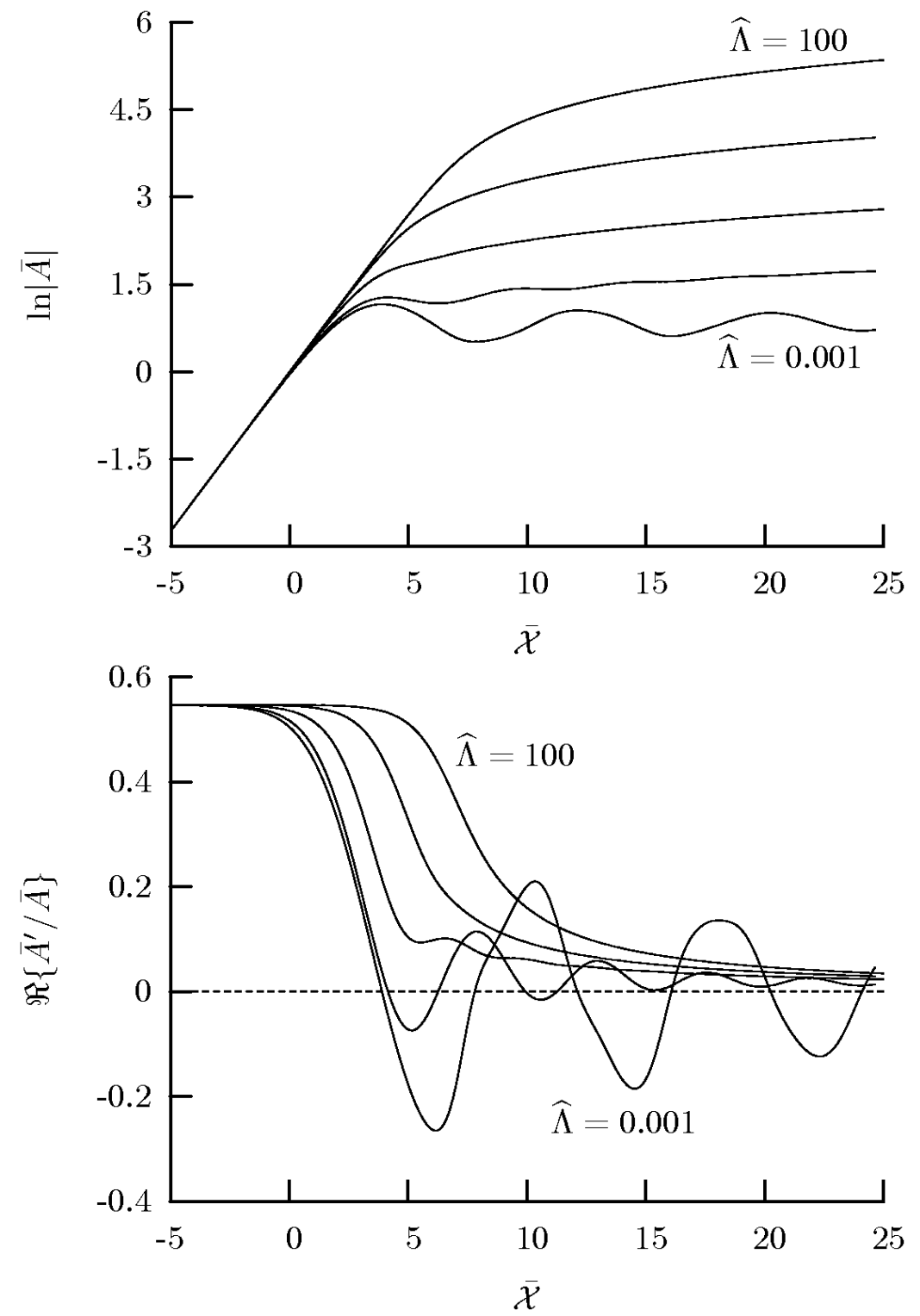

Figure 4: Amplitude $\ln |\bar{A}|$ (top) and instantaneous growth rate $\Re\left\{\bar{A}^{\prime} / \bar{A}\right\}$ (bottom) vs. $\overline{\mathcal{X}}$ for $\beta_{T}=1, M=1 / 2$ and $\widehat{\Lambda}=0.001,0.1,1,10,100$. 

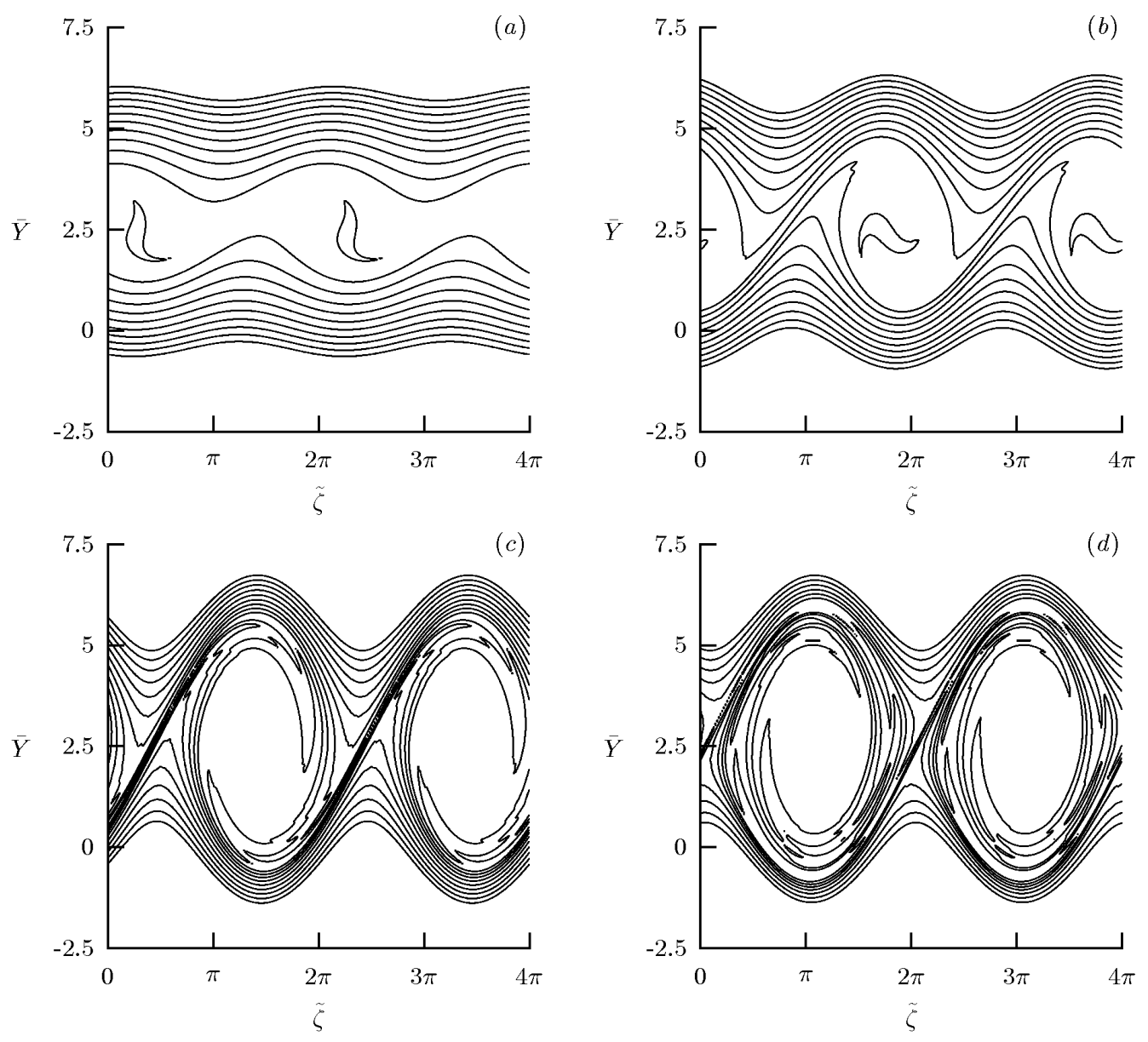

Figure 5: Vorticity contours in the $(\tilde{\zeta}, \bar{Y})$-plane for $\beta_{T}=1, M=1 / 2, \widehat{\Lambda}=0.001$ : $(a)$ $\overline{\mathcal{X}}=-0.986 ;(b) \overline{\mathcal{X}}=0.986 ;(c) \overline{\mathcal{X}}=2.958 ;(d) \overline{\mathcal{X}}=4.930$. (Contour levels shown are 0, $1,2, \ldots, 9$ and 10.)

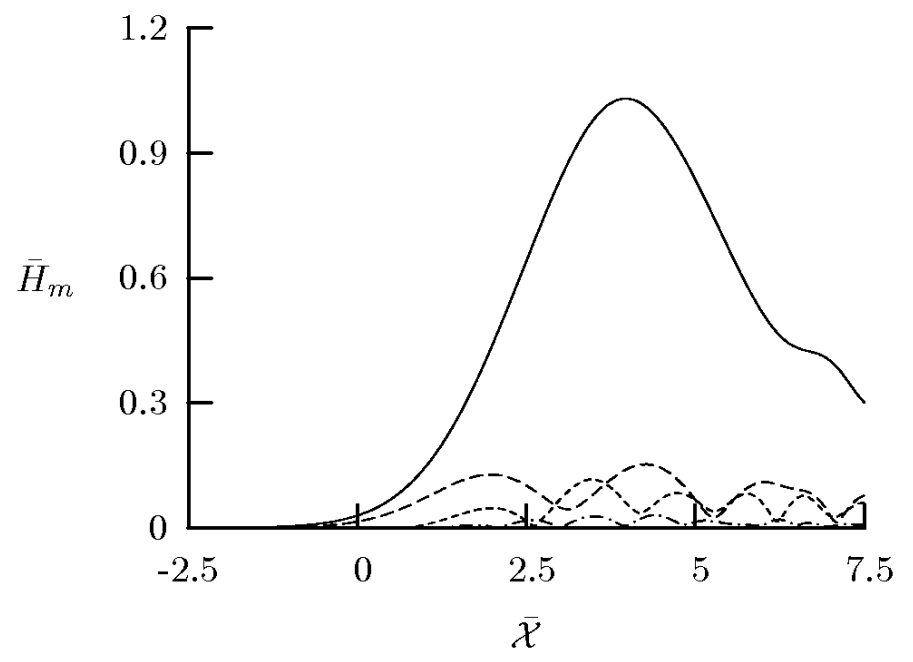

Figure 6: Development of higher harmonics, as measured by $\bar{H}_{m}$ (see (5.18)) vs. $\overline{\mathcal{X}}: m=2$ (solid), $m=3$ (long-dashed), $m=5$ (short-dashed) and $m=10$ (dash-dotted). 

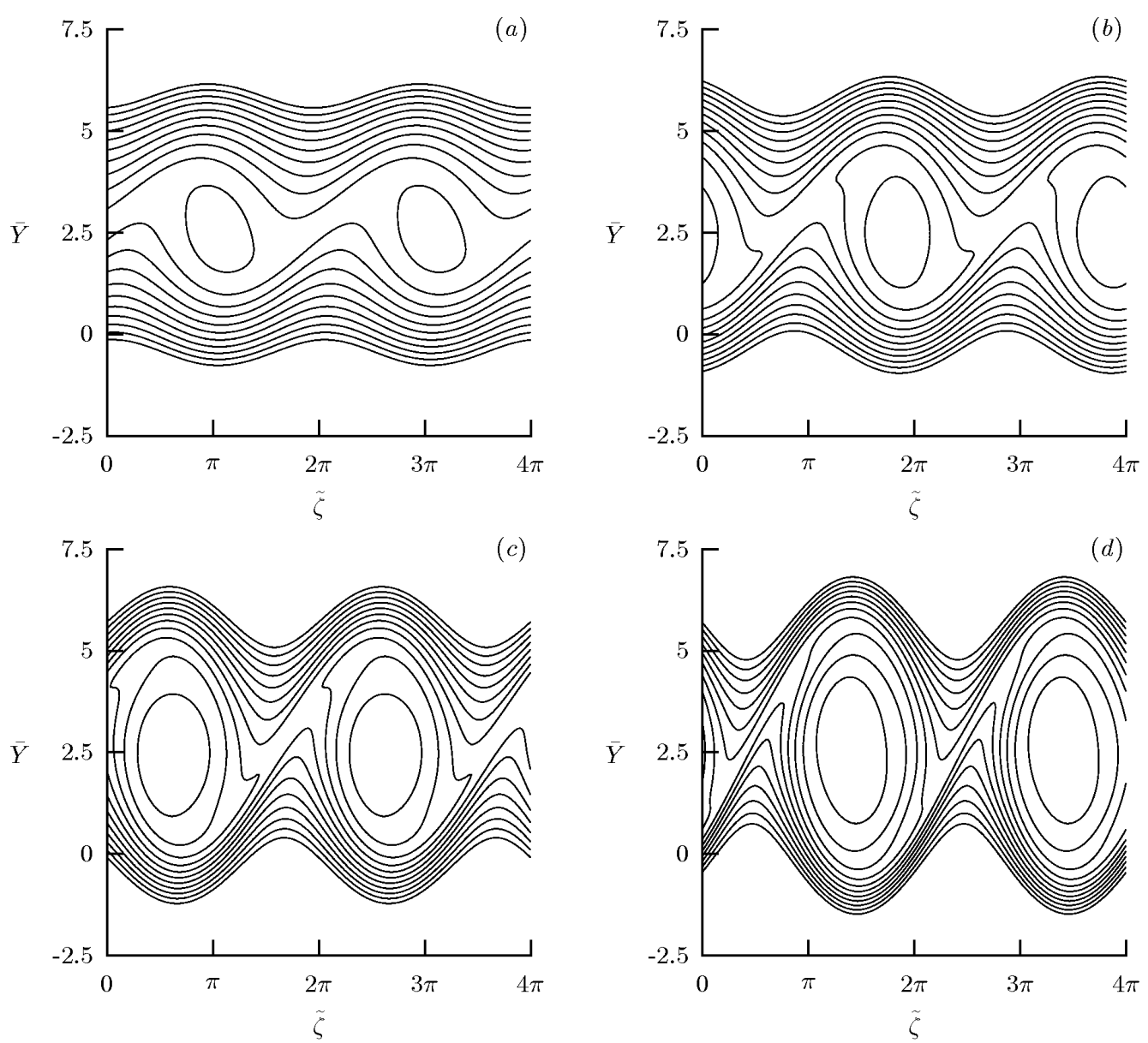

Figure 7: Vorticity contours in the $(\tilde{\zeta}, \bar{Y})$-plane for $\beta_{T}=1, M=1 / 2, \widehat{\Lambda}=0.1$ : $(a)$ $\overline{\mathcal{X}}=-0.986 ;(b) \overline{\mathcal{X}}=0.986 ;(a) \overline{\mathcal{X}}=2.958 ;(b) \overline{\mathcal{X}}=4.930$. 

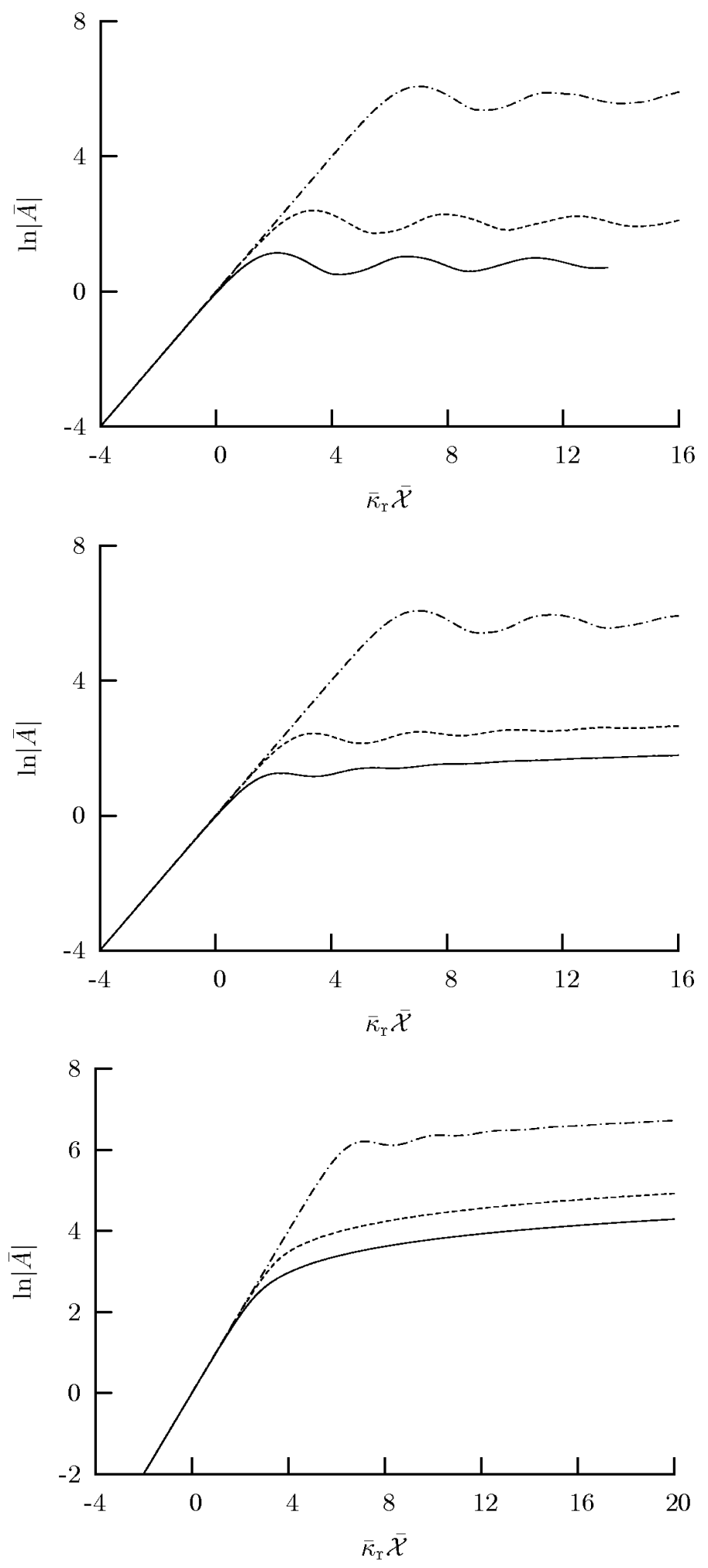

Figure 8: Amplitude $\ln |\bar{A}|$ vs. $\bar{\kappa}_{\mathrm{r}} \overline{\mathcal{X}}$ for $\beta_{T}=1$, and $M=0.1$ (solid), 3 (dashed), 3.96 (dash-dotted). Top: $\widehat{\Lambda}=0.001$; middle: $\widehat{\Lambda}=0.1$; bottom: $\widehat{\Lambda}=10$. 
Vorticity roll-up is the most prominent feature of mixing-layer transition. Figure 5 shows vorticity contours for $\widehat{\Lambda}=0.001$, which corresponds to a high Reynolds number $R=6.4 \times 10^{7}$, well beyond the range where DNS is possible. There is a great degree of roll-up, with mixing occurring mostly along the "braids", where the contours are closely clustered. The contours of the temperature field are almost 'isomorphic' to the vorticity contours in figure 5 and hence not shown separately. This close similarity may be explained by noting that since $\overline{\mathcal{M}}=0$ and $\left|\bar{T}_{m}\right| \ll\left|\bar{Q}_{m}\right|$, the temperature and vorticity equations, (4.19) and (4.20), are essentially the same.

Physically, roll-up is associated with the development of higher harmonics, which are simultaneously generated by nonlinear interactions. Figure 6 shows some of the harmonics, measured by (cf. Goldstein \& Leib 1988)

$$
\bar{H}_{m}=\int_{-\infty}^{\infty} \bar{Q}_{m} \mathrm{~d} \bar{Y}
$$

for the case of $\widehat{\Lambda}=0.001$. As is illustrated, the first harmonic $m=2$ has the most significant magnitude. Higher harmonics gradually become smaller, but the first a few harmonics (e.g. $m \leq 5)$ retain appreciate amplitudes.

For a moderate $\widehat{\Lambda}=0.1$, or Reynolds number $\left(R=6.4 \times 10^{5}\right)$, vorticity contours feature a regular cat's eye structure of Kelvin, and there is almost no roll-up (figure 7).

Overall, the nonlinear instability characteristics are nearly identical to the incompressible case of Goldstein \& Hultgren (1988). This is not surprising, since $T_{c}^{\prime}=0$ (for the tanh profile) and $M^{2}$ is quite small in the numerical sense so that the governing equations are very close to those for the incompressible case.

Next, we examine the effect of Mach number. Figure 8 shows the amplitude development for $\beta_{T}=1$ and various $M$ for three different $\widehat{\Lambda}$. The value $M=3.96$ is chosen to be $99 \%$ of $M_{\max }$, the maximum Mach number for subsonic modes to exist (see figure $3)$. For each $\widehat{\Lambda}$, increasing $M$ delays the onset of the nonlinear stage. The amplitude attains a larger value before it attenuates. Recall that in the incompressible case, the amplitude evolves into an asymptotic state $|\bar{A}| \sim(\bar{\Lambda} \overline{\mathcal{X}})^{2 / 3}$ (Goldstein \& Hultgren 1988). That a similar algebraic growth occurs is confirmed by re-plotting $\ln |\bar{A}|$ against $\ln \left|\kappa_{r} \overline{\mathcal{X}}\right|$. Straight lines of slope 2/3 are approached at large distances (Sparks 2006), indicating that the ultimate asymptotic state does not depend on Mach number for $\beta_{T}=1$. However, different large-distance behaviours are possible when $\beta_{T} \neq 1$; see $\S 6.6$.

Figures 9 and 10 show the vorticity and temperature contours for the case $M=3$ and $\widehat{\Lambda}=0.001$. In this fully compressible regime, roll-up remains a prominent feature, consistent with experimental observations (e.g. Clemens \& Mungal 1995, Olsen \& Dutton 2003). Indeed, both contour plots look similar to those for the low-Mach-number case $M=1 / 2$ (cf. figures 5 ); the difference is that vortex roll-up occurs further downstream as $M$ increases. As in the incompressible limit, increasing $\widehat{\Lambda}$ reduces roll-up.

\subsection{Case II: $\beta_{T} \neq 1$}

Next we turn to cases where the two streams have different temperatures, i.e. $\beta_{T} \neq 1$. A low Mach number $M=1 / 2$ is considered first. Figure 11 shows development of the amplitude for $\beta_{T}=1 / 2$ and a range of $\widehat{\Lambda}$. In this case, $\left|T_{c}^{\prime}\right| \neq 0$ and consequently the results contrast greatly with those for $\beta_{T}=1$. For small $\widehat{\Lambda}$ (e.g. 0.001 and 0.1 ), the amplitude exhibits a quite "chaotic" behaviour with growth rate oscillating violently, 

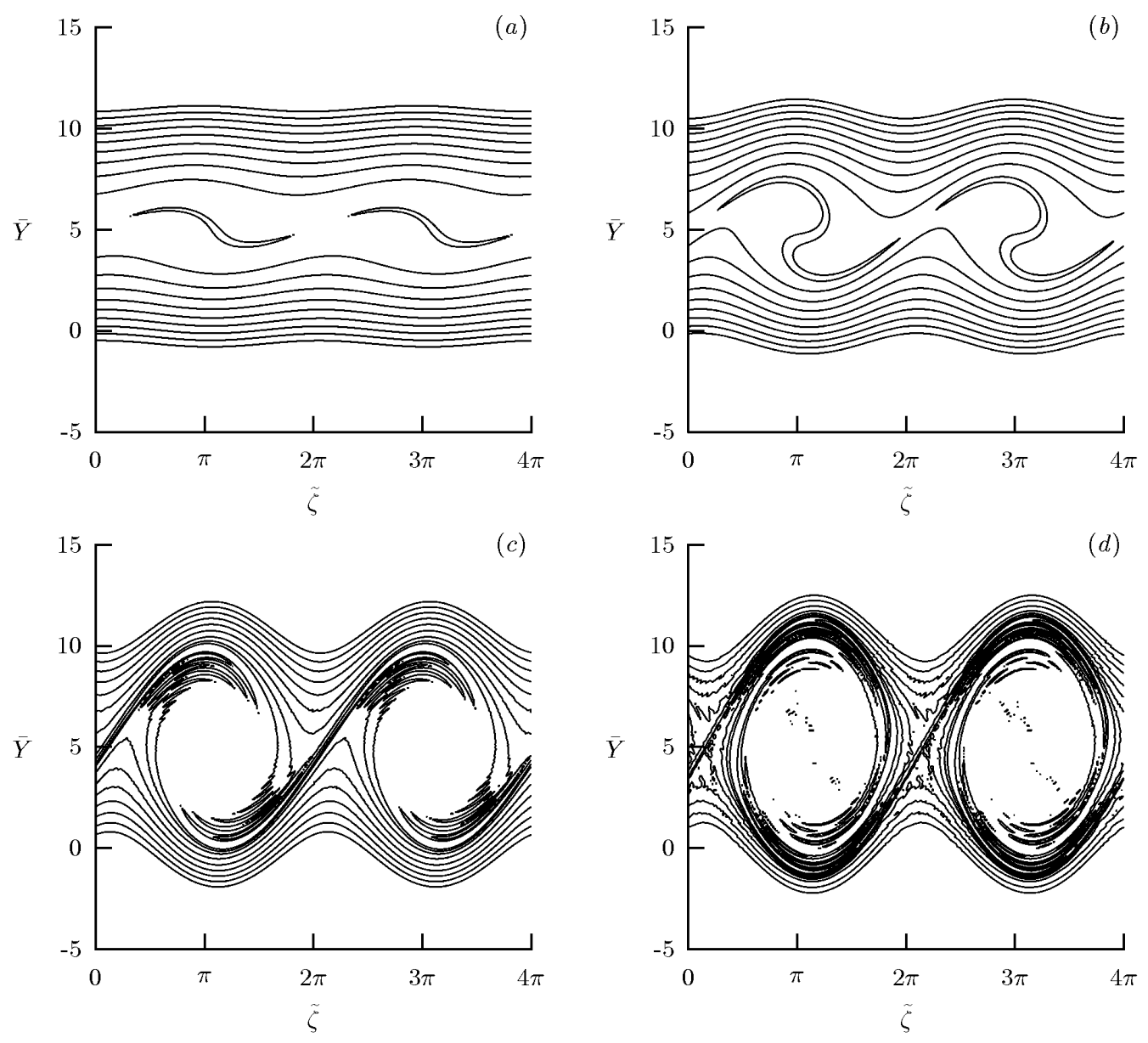

Figure 9: Vorticity contours in the $(\tilde{\zeta}, \bar{Y})$-plane for $\beta_{T}=1, M=3$ and $\widehat{\Lambda}=0.001$ : $(a) \bar{\kappa}_{\mathrm{r}} \overline{\mathcal{X}}+\ln \left(\alpha^{2}\right)=-1.325 ;(b) \bar{\kappa}_{\mathrm{r}} \overline{\mathcal{X}}+\ln \left(\alpha^{2}\right)=-0.101 ;(c) \bar{\kappa}_{\mathrm{r}} \overline{\mathcal{X}}+\ln \left(\alpha^{2}\right)=1.123 ;(d)$ $\bar{\kappa}_{\mathrm{r}} \overline{\mathcal{X}}+\ln \left(\alpha^{2}\right)=2.347$. (Contour levels shown are $0,3.75,7.5, \ldots, 30$ and 33.75.) 

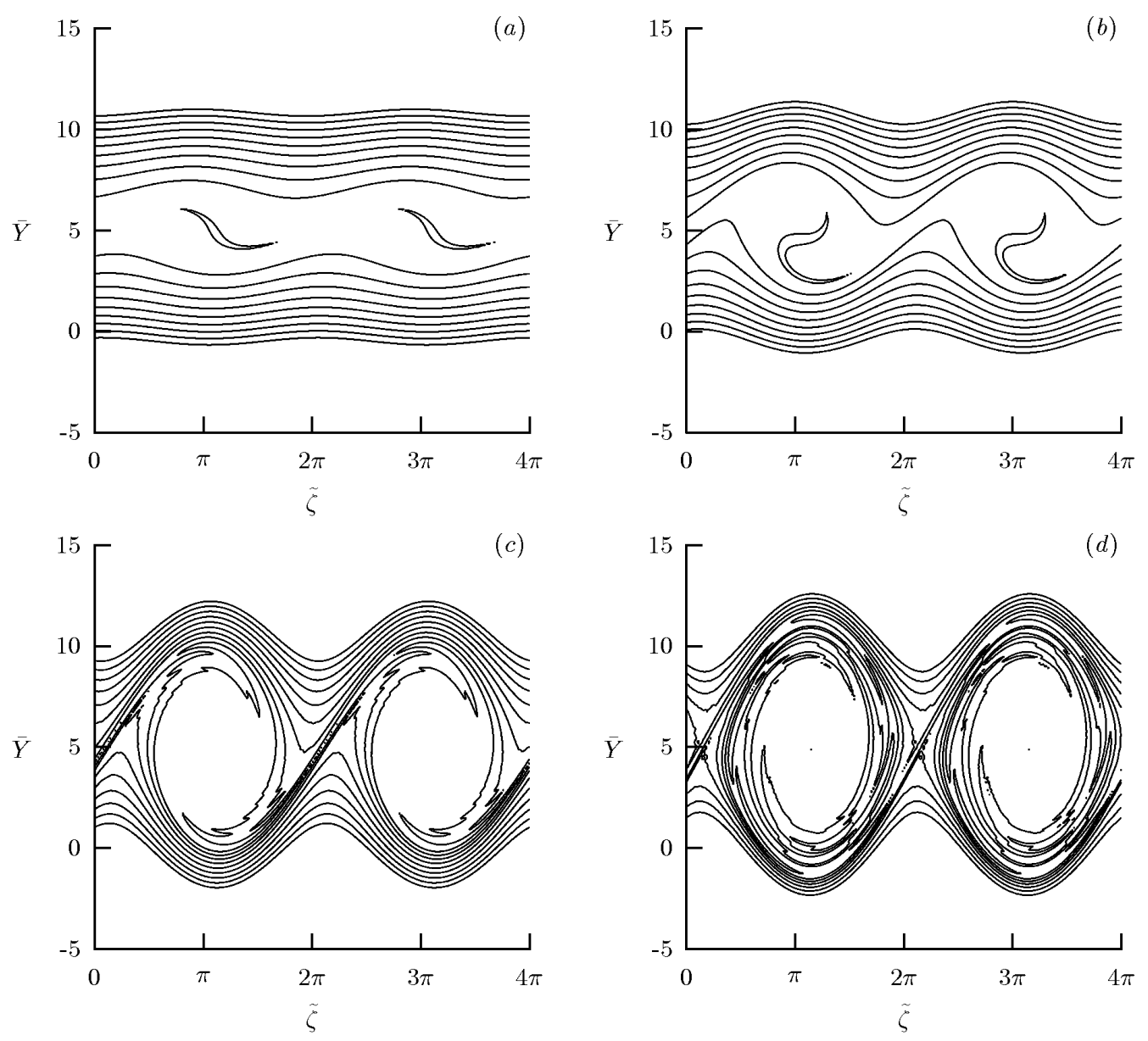

Figure 10: Temperature contours in the $(\tilde{\zeta}, \bar{Y})$-plane for $\beta_{T}=1, M=3$ and $\widehat{\Lambda}=0.001$ : (a) $\bar{\kappa}_{\mathrm{r}} \overline{\mathcal{X}}+\ln \left(\alpha^{2}\right)=-1.325 ;(b) \bar{\kappa}_{\mathrm{r}} \overline{\mathcal{X}}+\ln \left(\alpha^{2}\right)=-0.101 ;(c) \bar{\kappa}_{\mathrm{r}} \overline{\mathcal{X}}+\ln \left(\alpha^{2}\right)=1.123 ;(d)$ $\bar{\kappa}_{\mathrm{r}} \overline{\mathcal{X}}+\ln \left(\alpha^{2}\right)=2.347$. (Contour levels shown are $-3.6,-3.2,-2.8, \ldots,-0.4$ and 0 .)

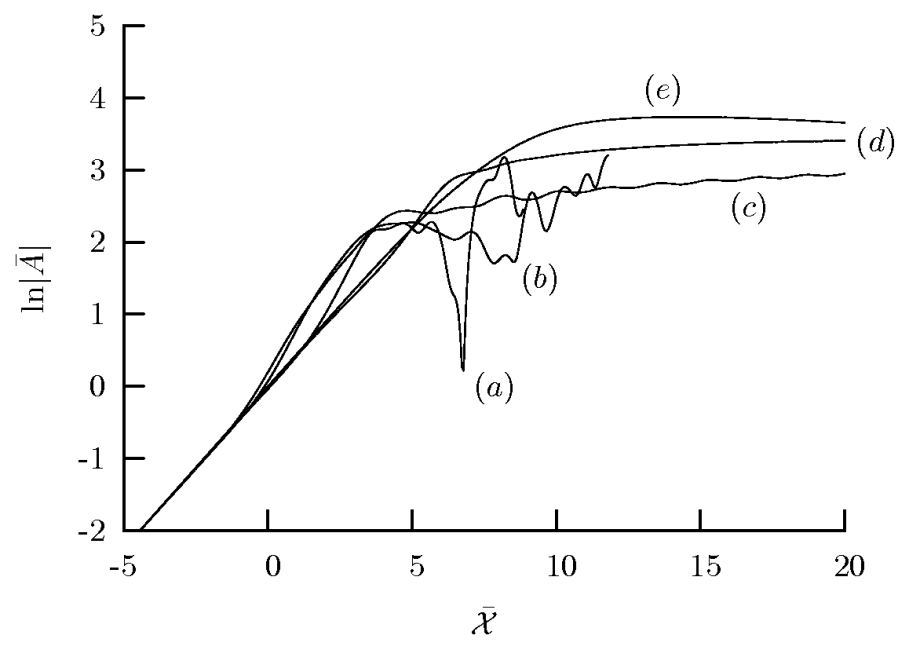

Figure 11: Amplitude $\ln |\bar{A}|$ vs. $\overline{\mathcal{X}}$ for $\beta_{T}=1 / 2, M=1 / 2$ : (a) $\widehat{\Lambda}=0.001 ;(b) \widehat{\Lambda}=0.1$; (c) $\widehat{\Lambda}=1 ;(d) \widehat{\Lambda}=10 ;(e) \widehat{\Lambda}=100$. 

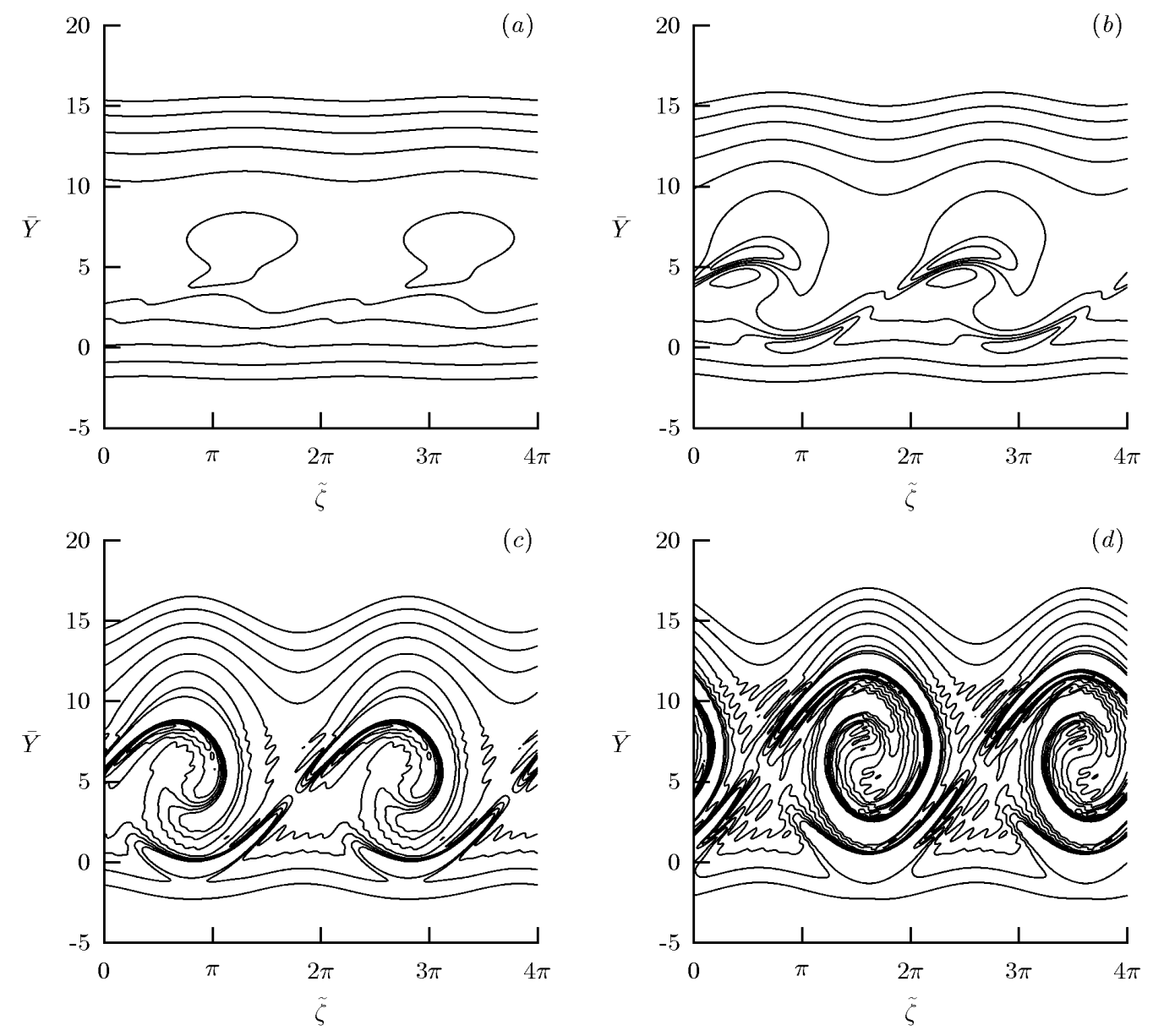

Figure 12: Vorticity contours in the $(\tilde{\zeta}, \bar{Y})$-plane for $\beta_{T}=1 / 2, M=1 / 2$ and $\widehat{\Lambda}=0.1:(a)$ $\overline{\mathcal{X}}=0 ;(b) \overline{\mathcal{X}}=1.475 ;(c) \overline{\mathcal{X}}=2.950 ;(d) \overline{\mathcal{X}}=4.425$. (Contour levels shown are -30 , $-15,0, \ldots, 60$ and 75.$)$

which is somewhat similar to that found in Goldstein \& Wundrow (1990). We were unable to advance the calculation arbitrarily far downstream, due to the difficulty of resolving small-scale structures appearing downstream. For moderate values of $\widehat{\Lambda}(\widehat{\Lambda}=1,10)$, solutions can be advanced far downstream, and the amplitude appears to grow slowly. For the largest value of $\widehat{\Lambda}(\widehat{\Lambda}=100)$ considered, the amplitude ultimately equilibrates.

Figure 12 shows vorticity contours for $\beta_{T}=1 / 2$ and $\widehat{\Lambda}=0.1$. Vorticity roll-up is much stronger than for $\beta_{T}=1$ shown in figure 7 . Closed "islands", similar to those found in Goldstein \& Wundrow (1990), soon appear. Further downstream, irregular small-scale motions cause contours to break up, forming tiny islands or excessive "wiggles". At the same time, temperature contours (figure 13) also wind up to form tight spirals, indicating a significant degree of mixing. Interestingly, they appear rather "regular", despite the fact that temperature roll-up is also a "cause" of vortex roll-up through coupling. A notable difference from the $\beta_{T}=1$ case is that entrainment now penetrates into the vortex cores. This feature appears to persist for all $\beta_{T} \neq 1$ (see below).

Further calculations performed by Sparks (2006) for smaller viscosity parameter $(\widehat{\Lambda}=$ 0.001) indicate that tighter spirals than those for $\widehat{\Lambda}=0.1$ can be observed. Apparently 

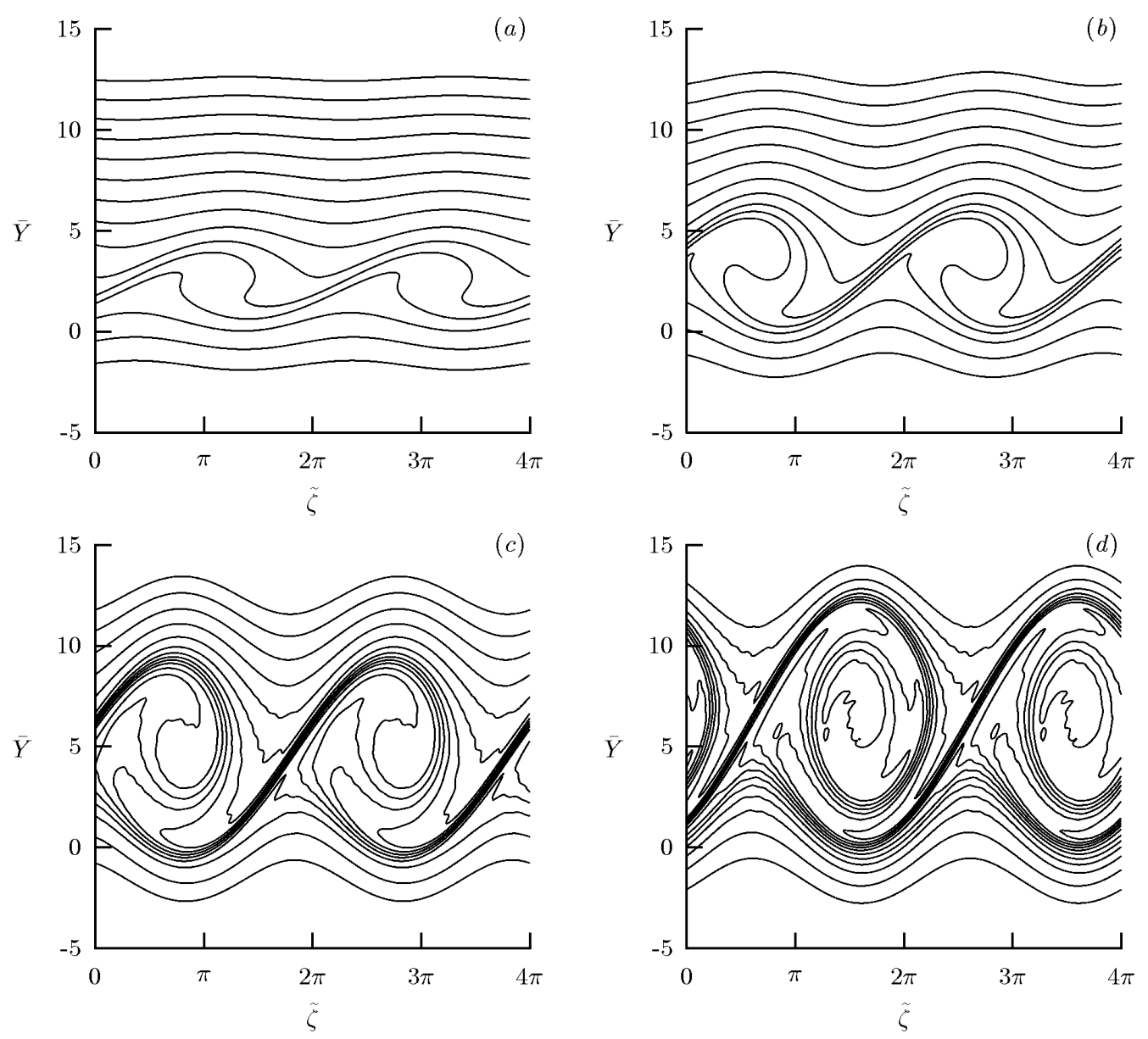

Figure 13: Temperature contours in the $(\tilde{\zeta}, \bar{Y})$-plane for $\beta_{T}=1 / 2, M=1 / 2$ and $\widehat{\Lambda}=0.1$ : $(a) \overline{\mathcal{X}}=0 ;(b) \overline{\mathcal{X}}=1.475 ;(c) \overline{\mathcal{X}}=2.950 ;(d) \overline{\mathcal{X}}=4.425$. (Contour levels shown are 370, $380,390, \ldots, 500$ and 510.) 

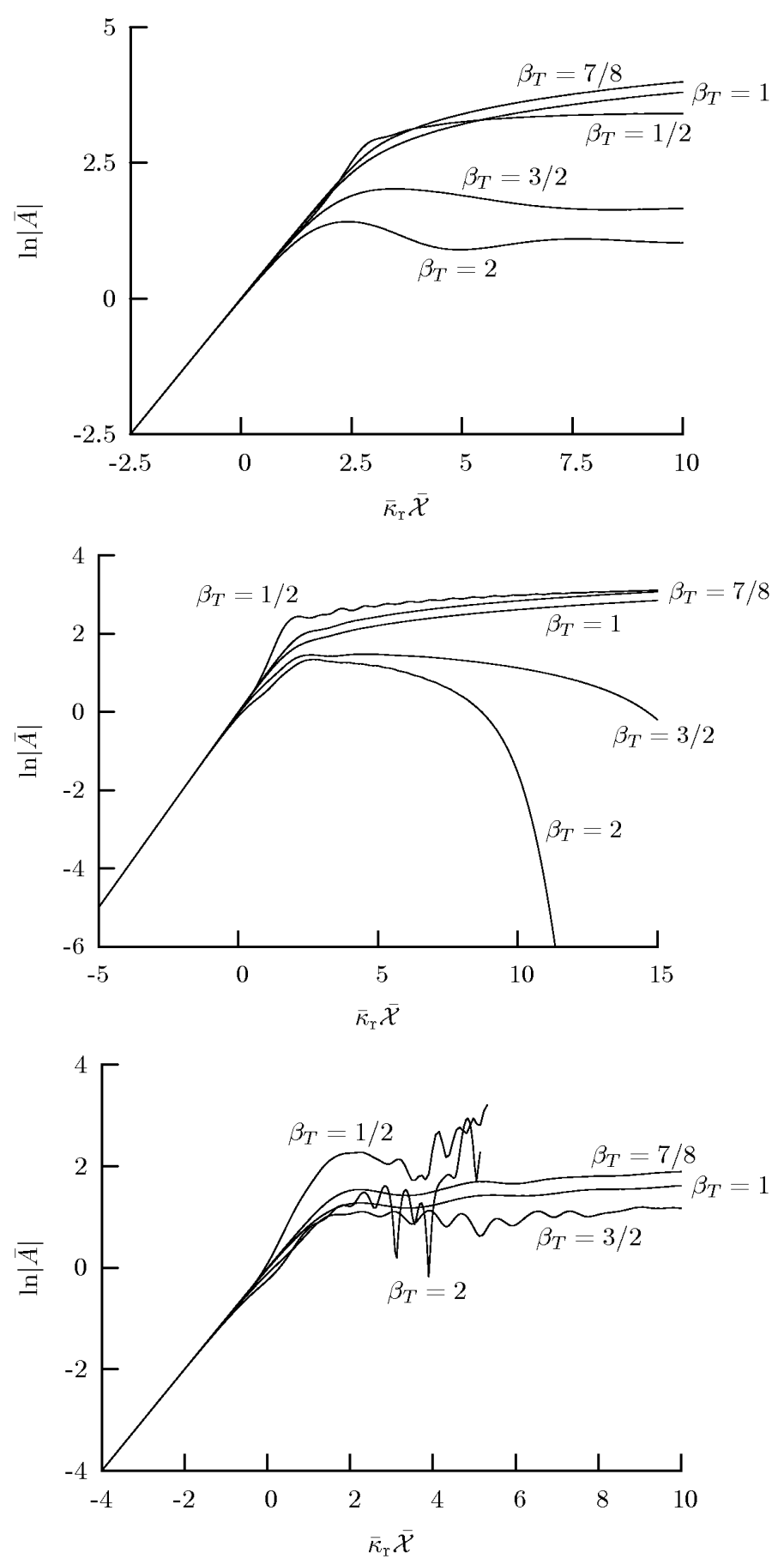

Figure 14: Amplitude development: $\ln |\bar{A}|$ vs. $\bar{\kappa}_{\mathrm{r}} \overline{\mathcal{X}}$ for $\beta_{T}=1 / 2,7 / 8,1,3 / 2,2 . \widehat{\Lambda}=10$ (top), 1 (middle), 0.1 (bottom). 

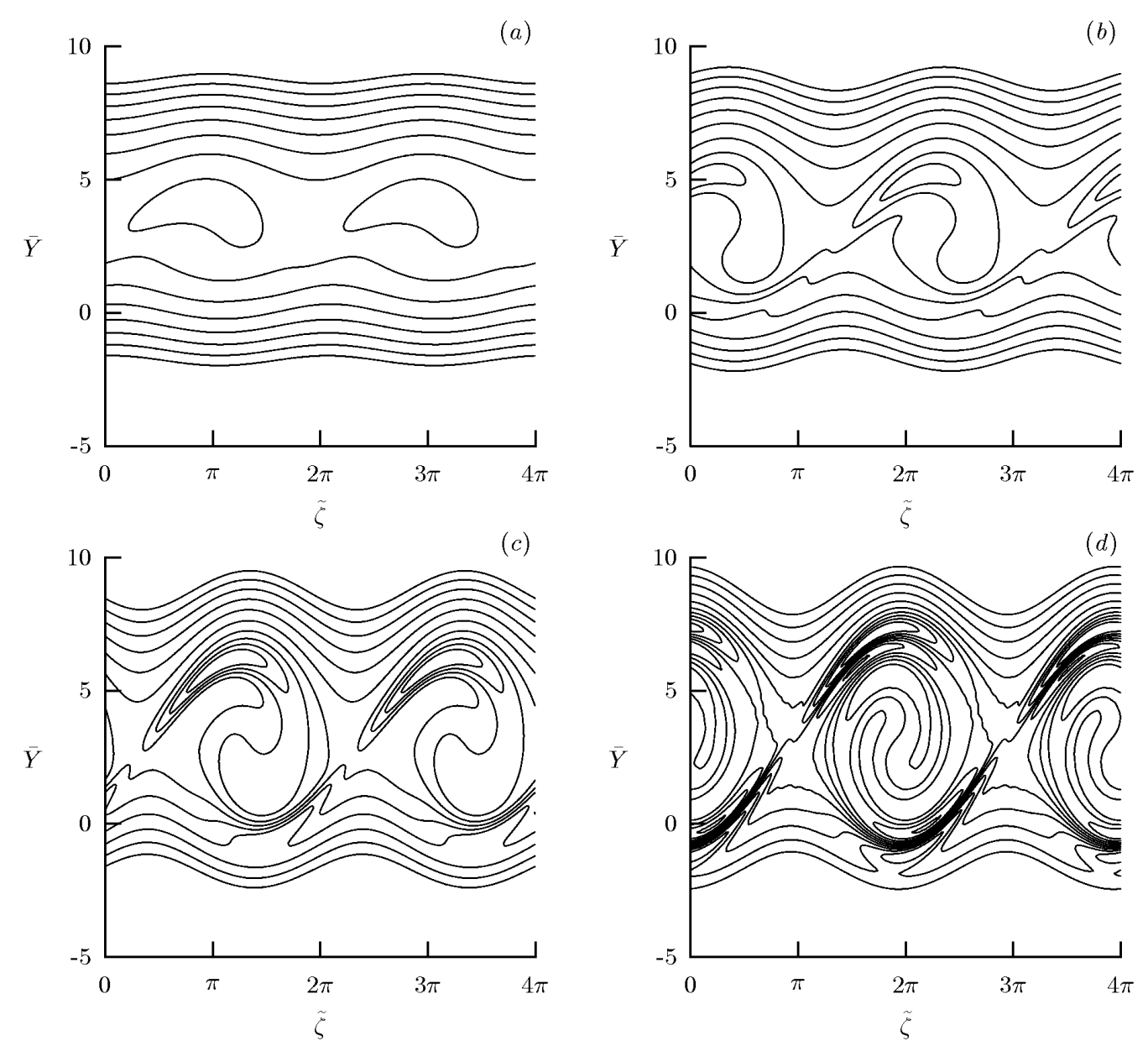

Figure 15: Vorticity contours in the $(\tilde{\zeta}, \bar{Y})$-plane for $\beta_{T}=7 / 8, M=1 / 2$ and $\widehat{\Lambda}=0.1$ : (a) $\bar{\kappa}_{\mathrm{r}} \overline{\mathcal{X}}=0 ;(b) \bar{\kappa}_{\mathrm{r}} \overline{\mathcal{X}}=0.861 ;(c) \bar{\kappa}_{\mathrm{r}} \overline{\mathcal{X}}=1.435 ;(d) \bar{\kappa}_{\mathrm{r}} \overline{\mathcal{X}}=2.869$. (Contour levels shown are $-8,-4,0, \ldots, 24$ and 28 .) 

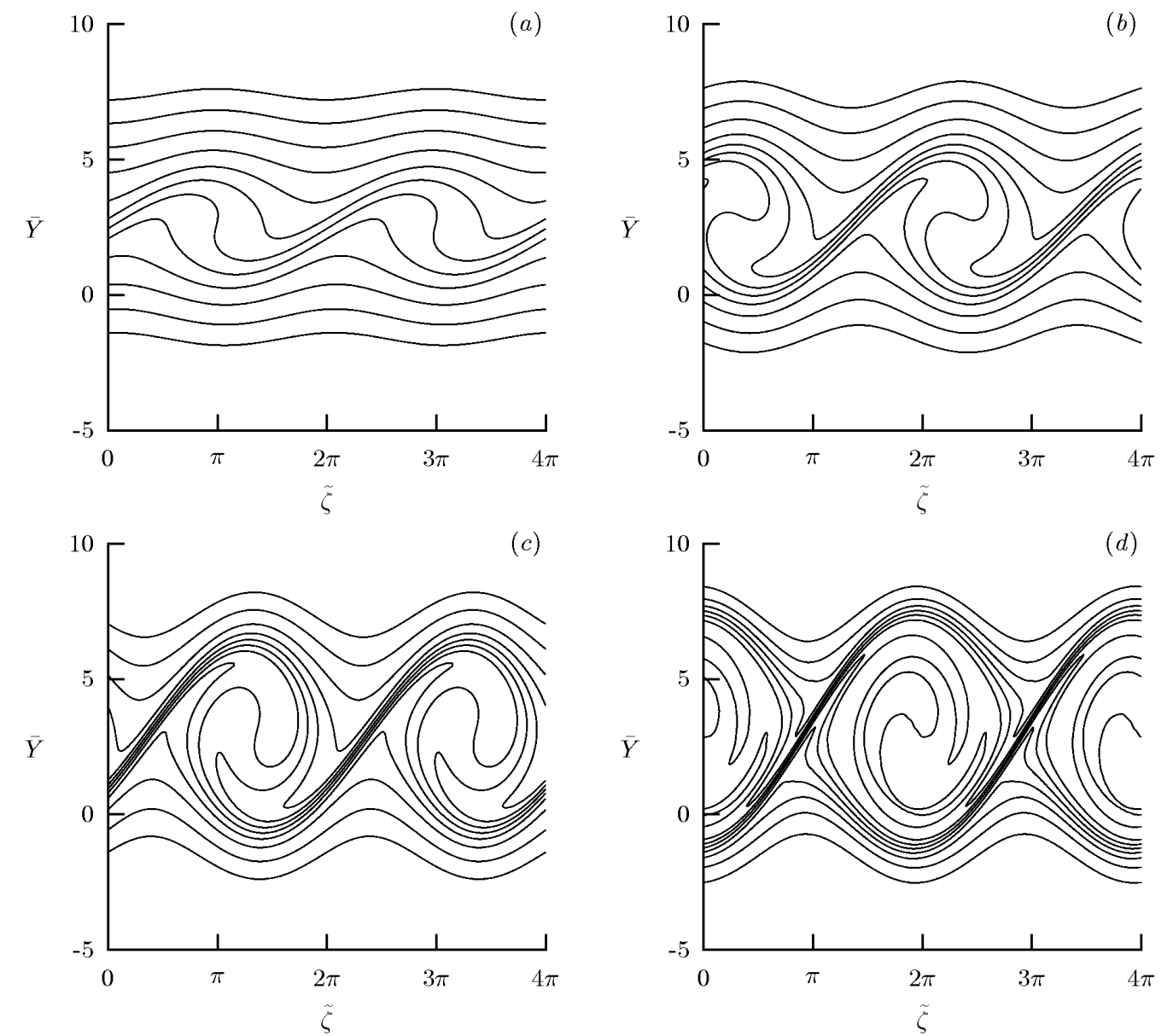

Figure 16: Temperature contours in the $(\tilde{\zeta}, \bar{Y})$-plane for $\beta_{T}=7 / 8, M=1 / 2$ and $\widehat{\Lambda}=0.1$ : $(a) \bar{\kappa}_{\mathrm{r}} \overline{\mathcal{X}}=0 ;(b) \bar{\kappa}_{\mathrm{r}} \overline{\mathcal{X}}=0.861 ;(c) \bar{\kappa}_{\mathrm{r}} \overline{\mathcal{X}}=1.435 ;(d) \bar{\kappa}_{\mathrm{r}} \overline{\mathcal{X}}=2.869$. (Contour levels shown are $-782.5,-781,-779.5, \ldots,-767.5$ and -766 .) 

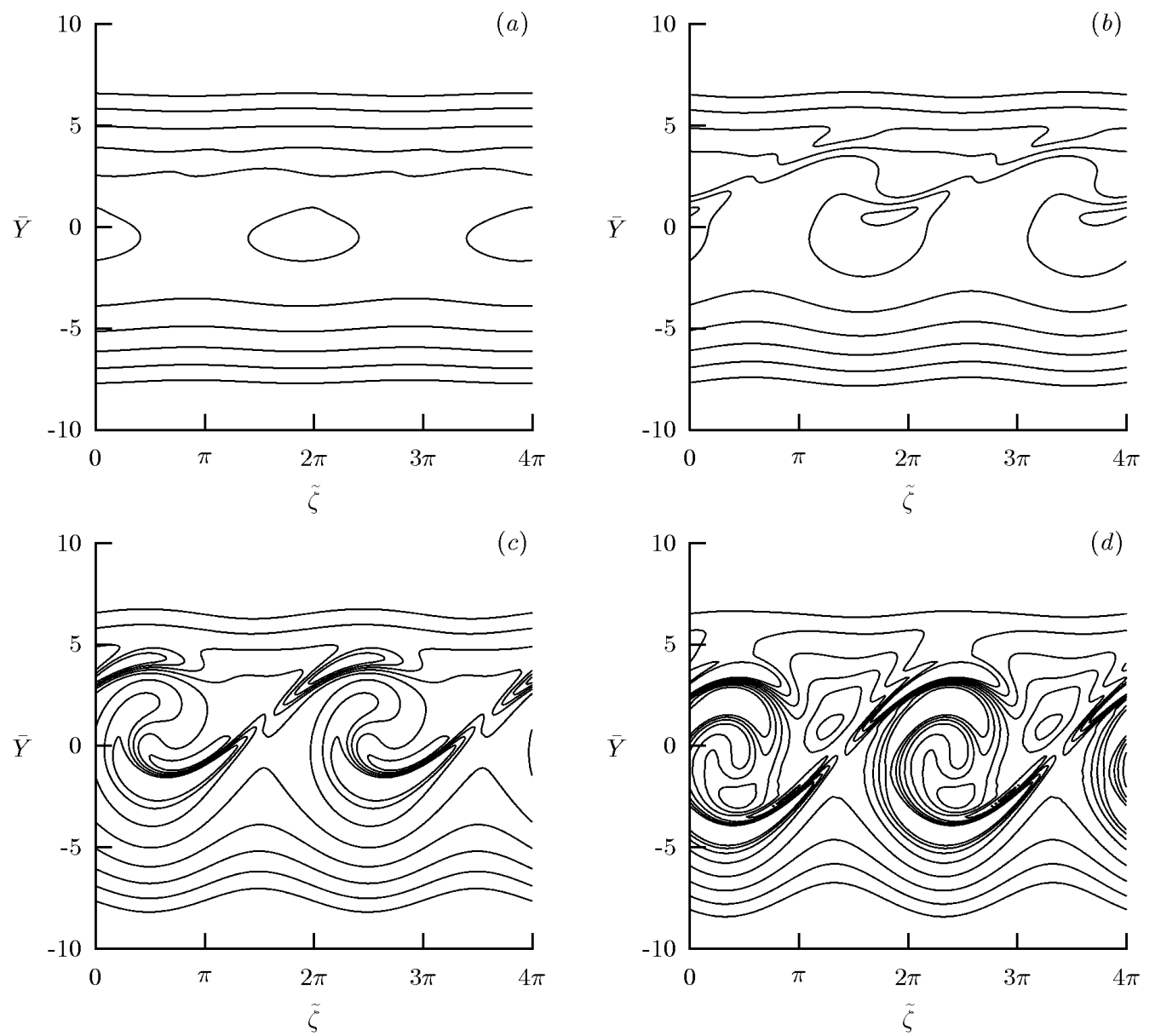

Figure 17: Vorticity contours in the $(\tilde{\zeta}, \bar{Y})$-plane for $\beta_{T}=2, M=1 / 2, \widehat{\Lambda}=0.1:(a)$ $\bar{\kappa}_{\mathrm{r}} \overline{\mathcal{X}}=-0.514 ;(b) \bar{\kappa}_{\mathrm{r}} \overline{\mathcal{X}}=0.514 ;(c) \bar{\kappa}_{\mathrm{r}} \overline{\mathcal{X}}=1.542 ;(d) \bar{\kappa}_{\mathrm{r}} \overline{\mathcal{X}}=2.571$. $\quad$ Contour levels shown are $-20,-10,0, \ldots, 40$ and 50.$)$ 

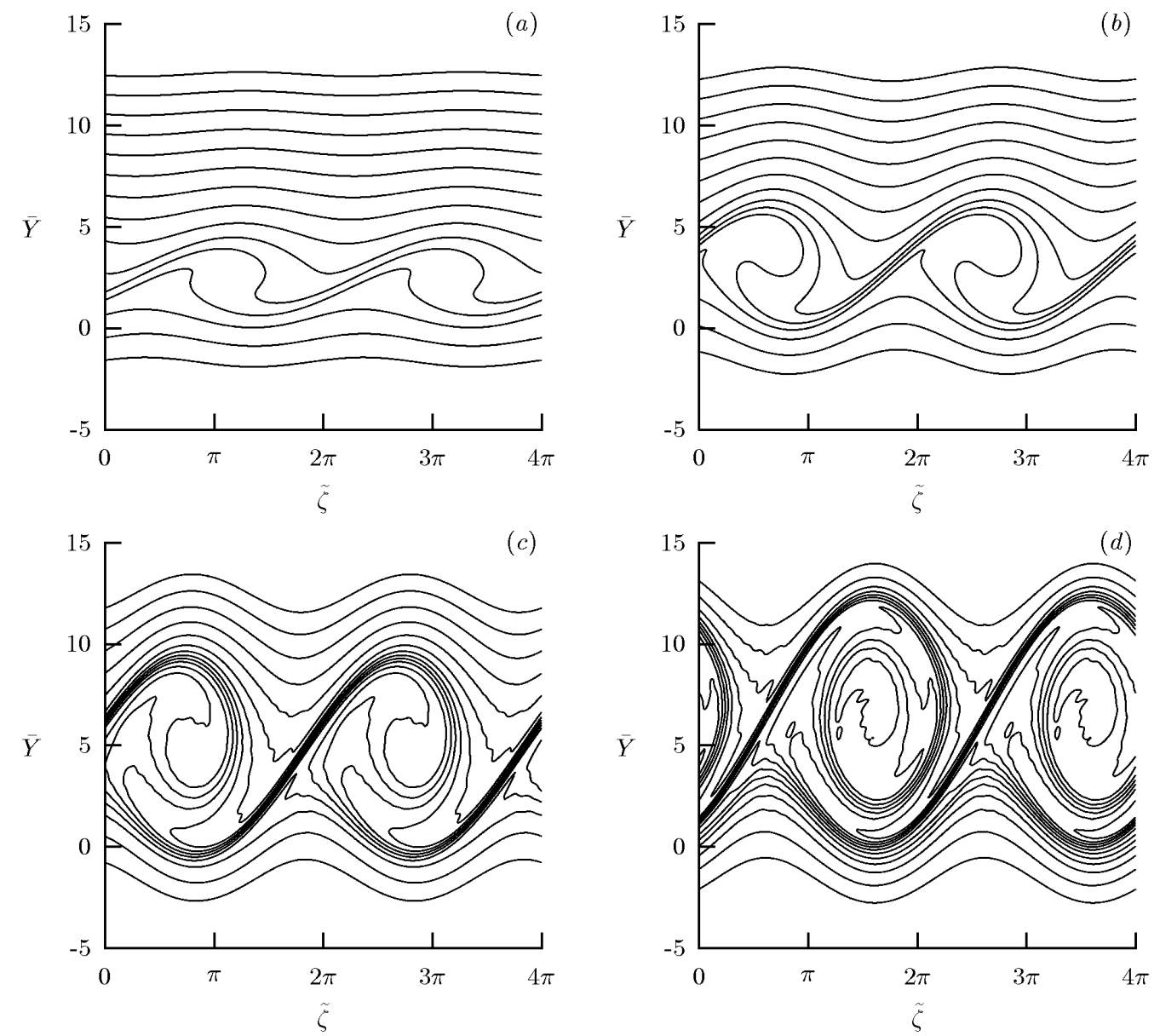

Figure 18: Temperature contours in the $(\tilde{\zeta}, \bar{Y})$-plane for $\beta_{T}=2, M=1 / 2$, and $\widehat{\Lambda}=0.1$ : $(a) \bar{\kappa}_{\mathrm{r}} \overline{\mathcal{X}}=-0.514 ;(b) \bar{\kappa}_{\mathrm{r}} \overline{\mathcal{X}}=0.514 ;(c) \bar{\kappa}_{\mathrm{r}} \overline{\mathcal{X}}=1.542 ;(d) \bar{\kappa}_{\mathrm{r}} \overline{\mathcal{X}}=2.571$. (Contour levels shown are 226, 234, 242, .., 322 and 330.) 
pathological small-scale motions become so severe that contours break up near the cores. An analysis of the development of harmonics shows that in contrast to the $\beta_{T}=1$ case, quite a few harmonics are of comparable magnitude, with higher harmonics overtaking the lower ones further downstream. This was always found to occur regardless of the number of Fourier components used (ranging from $m=5$ to $m=100$ ), suggesting that the increasingly vigorous small-scale motions cannot be resolved by the present spectral method. Nevertheless, numerical tests show that the gross feature of the larger-scale structure apparently remains intact. It may be noted that when the flow field becomes dominated by motions with a streamwise length scale comparable with the critical-layer thickness, the critical-layer theory ceases to be valid.

The effect of $\beta_{T}$ on the amplitude could, depending on $\widehat{\Lambda}$, be rather complicated, as shown in figure 14. For a moderate or relatively large $\widehat{\Lambda}(\widehat{\Lambda} \geqslant 1)$, increasing $\beta_{T}$ above unity has a stabilising effect. In particular, for $\widehat{\Lambda}=10$ the amplitude equilibrates rather than undergoing (quasi-)algebraic growth as in the case of $\beta_{T}=1$. For $\widehat{\Lambda}=1$, the amplitude decays, but a further examination (Sparks 2006) shows it remains at an almost zero level for a prolonged period and then would re-emerge again. This cycle of extinction and resurrection repeats itself in a quasi-periodic fashion. Decreasing $\beta_{T}$ below unity has a relatively moderate effect on the amplitude for relatively large $\widehat{\Lambda}$. For small $\widehat{\Lambda}$ (e.g. $\widehat{\Lambda} \leqslant 0.1$ ), increasing $\beta_{T}$ above unity or decreasing $\beta_{T}$ below unity causes the amplitude to undergo increasingly complex oscillation; see the bottom plot of figure 14 .

Vorticity and temperature contours are displayed in figures 15 and 16 respectively for the case $\widehat{\Lambda}=0.1$ and $\beta_{T}=7 / 8$. Interestingly, although the amplitude development is very similar to the $\beta_{T}=1$ case, vorticity contours appear quite different in that there is now vigorous roll-up (cf. figure 7 ). On the other hand, compared to the $\beta_{T}=1 / 2$ case (figures 12 and 13), roll-up is not as strong, and contours do not consist of irregular wiggles or tiny islands, suggesting that small-scale motions do not develop in this case.

As a contrast with the $\beta_{T}=1 / 2$ case, figures 17 and 18 show vorticity and temperature contours for $\beta_{T}=2$, i.e. the slower stream is hotter. Mixing appears to be somewhat less strong than in the "opposite" case $\beta_{T}=1 / 2$ (cf. figures 12 and 13), suggesting that mixing would be relatively improved by cooling the slower stream.

We now examine the effect of Mach number for a non-unity $\beta_{T}=1 / 2$. Figure 19 shows the amplitude development for various $M$, where $M=2.87$ is chosen to be $99 \%$ of $M_{\max }$. Unlike the $\beta_{T}=1$ case shown in Figure 8, the effect of $M$ now depends on $\widehat{\Lambda}$. For $\widehat{\Lambda}=1$, the solution for $M=2.87$ rapidly decays. The solutions for $M=0.1$ and $M=2$ appear to attenuate slowly, but when advanced further downstream, they exhibit a slow decay. Thus for this value of $\widehat{\Lambda}$, increasing $M$ has an appreciable stabilising effect. For $\widehat{\Lambda}=0.1$, the amplitude is reduced initially as $M$ increases, but thereafter strong oscillations occur, and the amplitude at higher $M$ turns out to be larger.

Vorticity and temperature contours are shown in figures 20 and 21 for $\beta_{T}=1 / 2$, $M=2.87$ and $\widehat{\Lambda}=0.1$. Roll-up and mixing are the most notable features, which cannot be predicted by weakly nonlinear theory. The contours look more-or-less similar to the nearly incompressible case (cf. figures 12 and 13) with an appreciable reduction in roll-up.

In summary, the numerical results demonstrate that mixing layer dynamics behave more or less the same as in the incompressible case even when $M$ is of order one and $\beta_{T}$ differs considerably from unity. The results presented in this paper are for velocity ratio $\beta_{U}=1 / 2$ and for the tanh profile. Further calculations were performed by Sparks (2006) for other values of $\beta_{U}$ and also for Lock profiles (obtained for both Chapman and Sutherland viscosity laws). It was found that the quantitative behaviour of the amplitude, 

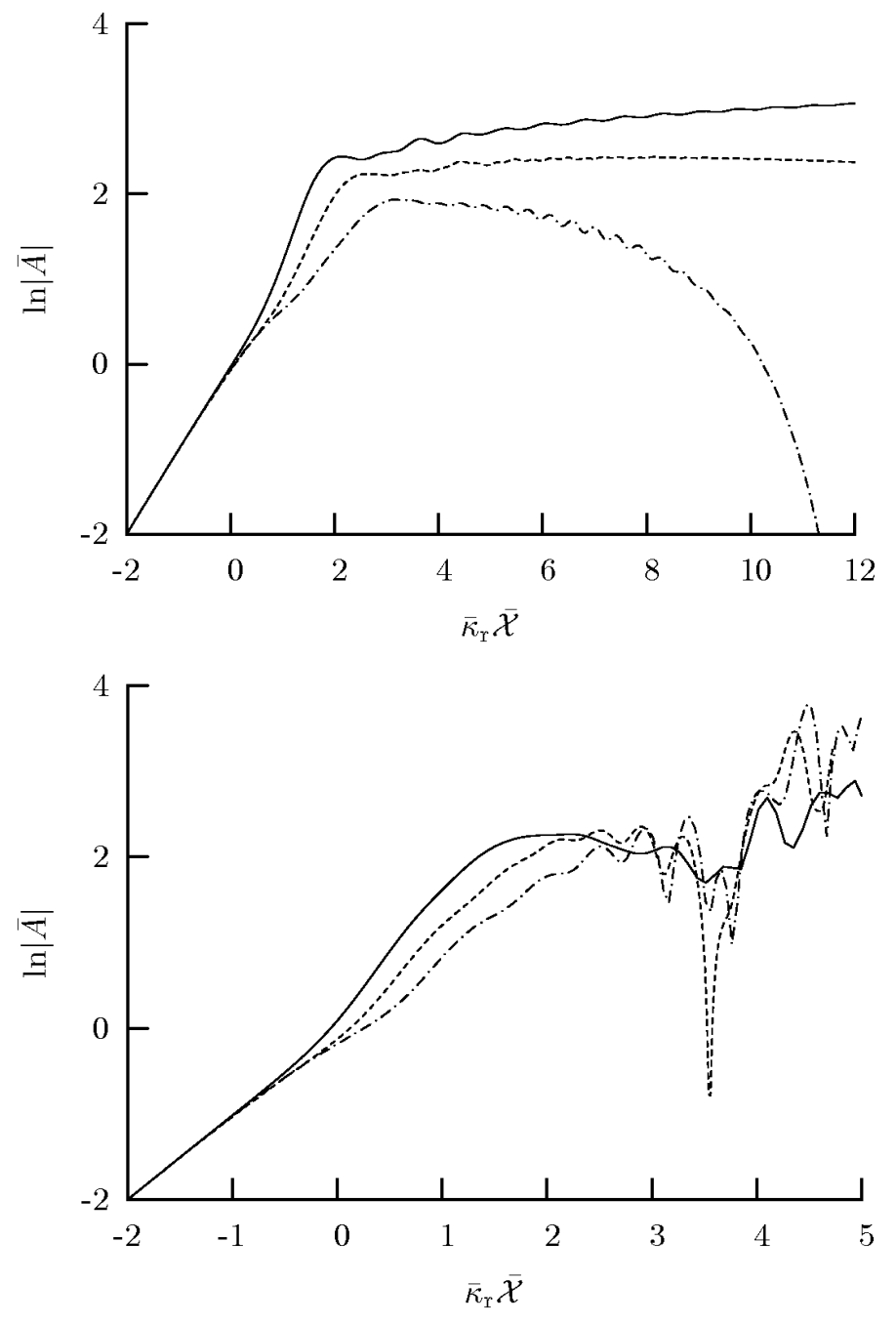

Figure 19: Amplitude $\ln |\bar{A}|$ vs. $\bar{\kappa}_{\mathrm{r}} \overline{\mathcal{X}}$ for $\beta_{T}=1 / 2$, and $M=0.1$ (solid), 2 (short-dashed), 2.87 (dash-dotted). $\widehat{\Lambda}=1$ (top), 0.1 (bottom). 

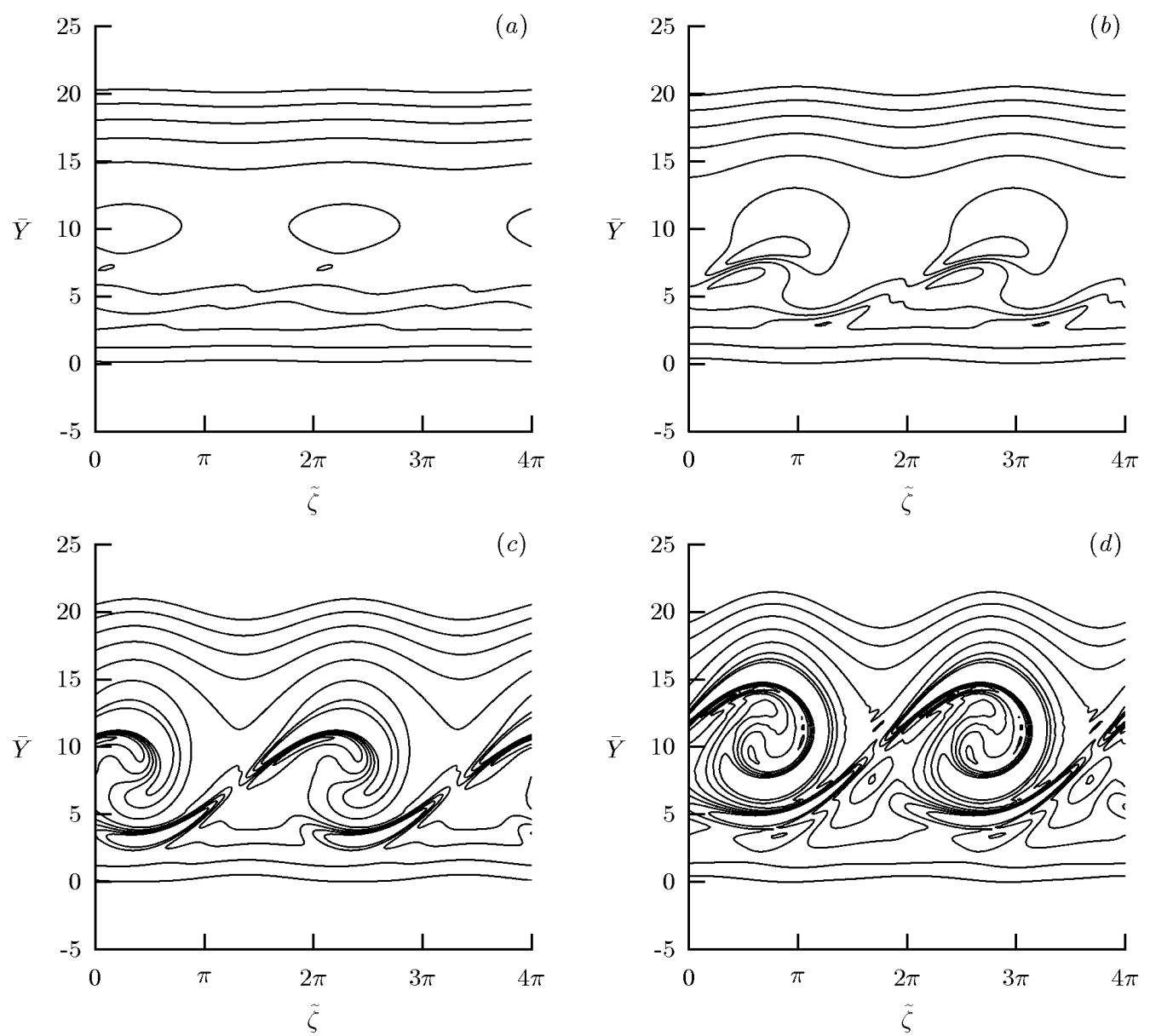

Figure 20: Vorticity contours in the $(\tilde{\zeta}, \bar{Y})$-plane for $\beta_{T}=1 / 2, M=2.87$ and $\widehat{\Lambda}=0.1$ : $(a) \bar{\kappa}_{\mathrm{r}} \overline{\mathcal{X}}=0.451 ;(a) \bar{\kappa}_{\mathrm{r}} \overline{\mathcal{X}}=1.352 ;(b) \bar{\kappa}_{\mathrm{r}} \overline{\mathcal{X}}=2.254 ;(c) \bar{\kappa}_{\mathrm{r}} \overline{\mathcal{X}}=3.156$. 

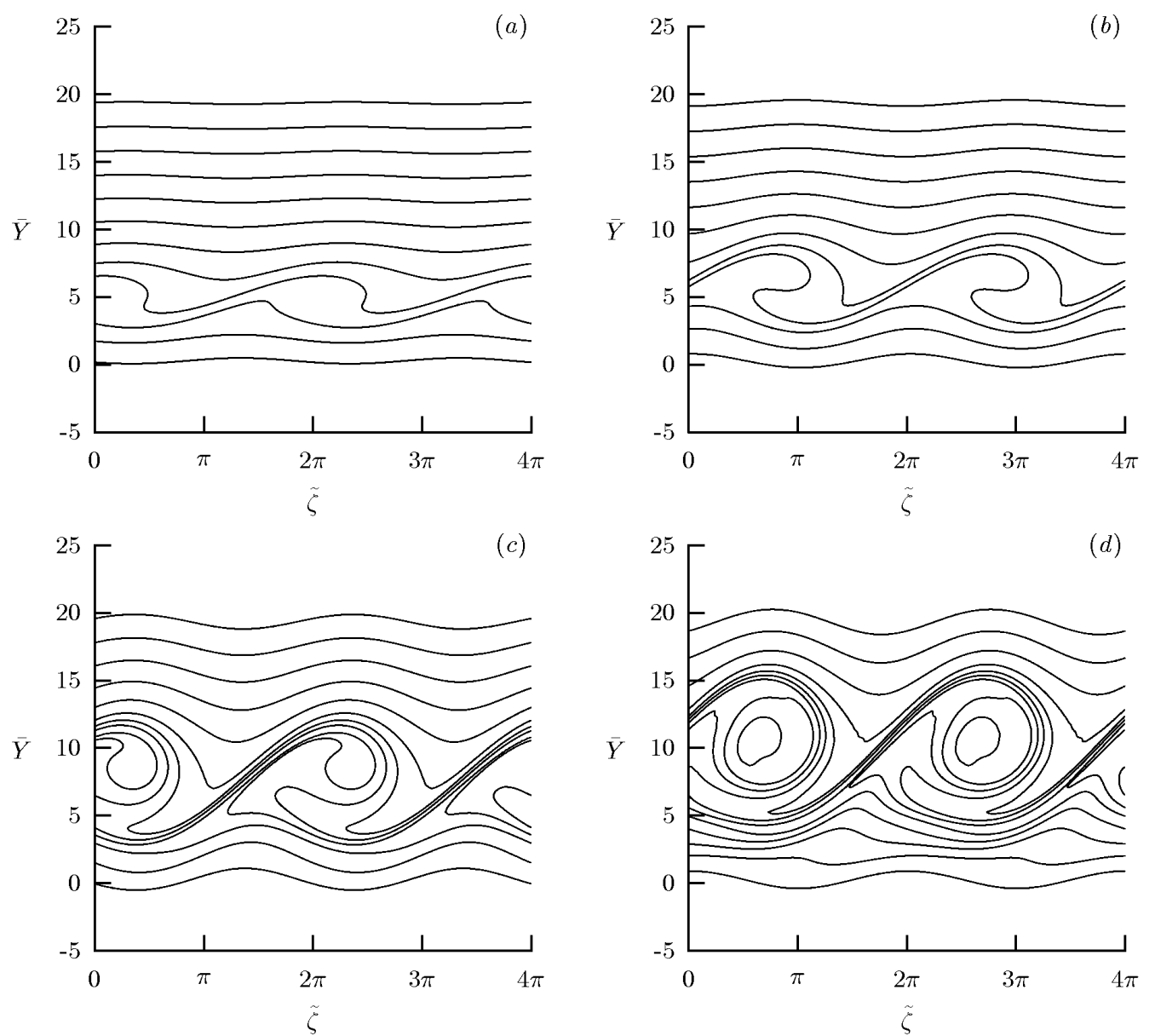

Figure 21: Temperature (right) contours in the $(\tilde{\zeta}, \bar{Y})$-plane for $\beta_{T}=1 / 2, M=2.87$ and $\widehat{\Lambda}=0.1:(a) \bar{\kappa}_{\mathrm{r}} \overline{\mathcal{X}}=0.451 ;(a) \bar{\kappa}_{\mathrm{r}} \overline{\mathcal{X}}=1.352 ;(b) \bar{\kappa}_{\mathrm{r}} \overline{\mathcal{X}}=2.254 ;(c) \bar{\kappa}_{\mathrm{r}} \overline{\mathcal{X}}=3.156$. 

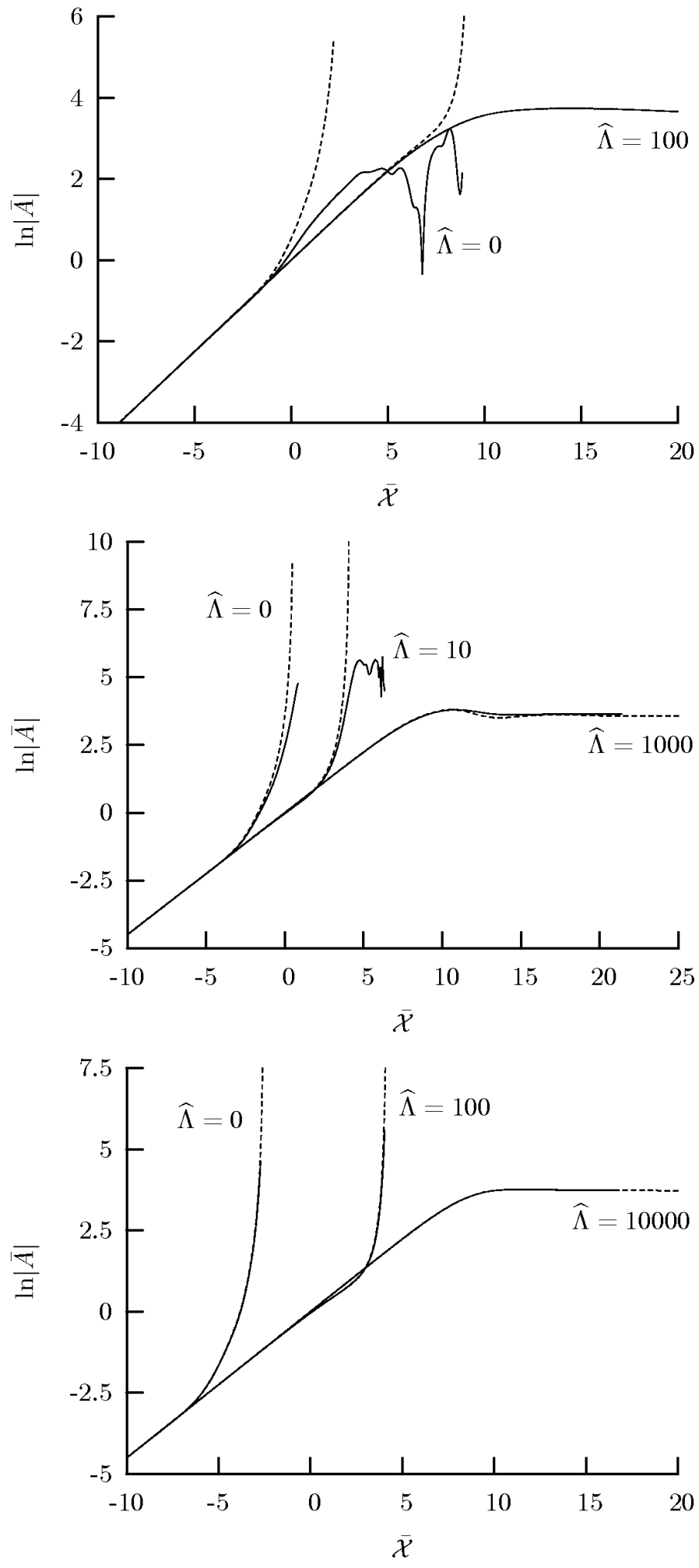

Figure 22: Amplitude $\ln |\bar{A}|$ vs. $\overline{\mathcal{X}}$ for $\beta_{T}=1 / 2$ and $M=1 / 2$. Dashed lines show equivalent weakly nonlinear results. $\hat{\omega}=1 / 40$ (top), $1 / 250$ (middle) and $1 / 4000$ (bottom). 
the vorticity and temperature remains similar provided that the instability is convective in its nature; the present spatial evolution theory does not apply to absolutely unstable regime. Within the strongly nonlinear formulation, the tanh profile is robust, without suffering from the degeneracy in the weakly nonlinear theory (cf. Leib 1991).

\subsection{Comparisons between strongly and weakly nonlinear predictions}

The weakly nonlinear amplitude equation (4.30) was formally derived from the strongly nonlinear system in the limit $\overline{\mathcal{M}} \gg 1$. The calculations presented in the previous sections focussed on $\overline{\mathcal{M}}^{2} \equiv T_{c}^{\prime} / \hat{\omega}=\mathcal{O}(1)$, for which (4.30) is not expected to give a good approximation. The validity of (4.30) is now examined by comparing its predictions with those of the strongly nonlinear theory for different $\hat{\omega}$ (and hence $\overline{\mathcal{M}}$; see $(5.17)$ ).

As an example, we consider the case where $\beta_{T}=1 / 2$ and $M=1 / 2$. Figure 22 shows comparisons for three different values of $\hat{\omega}$. For $\hat{\omega}=1 / 40$, the value used in the calculations presented above, the results from the two theories overlap in the linear regime only, and deviate immediately once the nonlinear effect becomes important. For $\hat{\omega}=1 / 250$, there is an appreciable overlap in the nonlinear regime, the extent of which depends on $\widehat{\Lambda}$. For small to moderate $\widehat{\Lambda}$, the weakly nonlinear solutions deviate eventually from the strongly nonlinear ones, whereas for large $\widehat{\Lambda}$, the two solutions are almost identical for all $\overline{\mathcal{X}}$. For an extremely small $\hat{\omega}=1 / 4000$, the two theories give essentially the same results. Sparks (2006) monitored harmonical contents, $\bar{H}_{m}$, for $m$ up to 5 , and found that $H_{m}$ decreases rapidly with $m$, satisfying a pre-request for weakly nonlinear theory to be valid. Meanwhile, vorticity was found to exhibit very little roll-up as a result.

The comparisons above (as well as those in Sparks (2006), where further calculations were performed for other values of $\beta_{T}$ and $M$ ) demonstrate that the weakly nonlinear theory yields, as expected, the same result as the strongly nonlinear theory provided that $\overline{\mathcal{M}}$ exceeds a large enough threshold. This requires a very small deviation from the neutrality, for typical large Reynolds numbers of practical relevance. Otherwise, the weakly nonlinear theory cannot predict the correct amplitude evolution, and in any case fails to describe vortex roll-up. Since the strongly nonlinear system can be routinely solved on PCs of moderate capacity, it should be the preferred framework for investigating the two-dimensional nonlinear instability of mixing layers.

\section{Discussion and conclusions}

In this paper, we carried out a theoretical investigation of the nonlinear instability of compressible mixing layers. Attention is focussed on the regime of low-to-moderate (but including supersonic) Mach numbers, where subsonic modes play a dominant role in causing transition. Our primary interest is in predicting the nonlinear evolution of these modes, which requires a careful analysis of critical layer dynamics. By considering the distinguished weakly compressible regime, we presented a theory which 'bridges' the strongly and weakly nonlinear critical-layer theories, which were formulated respectively for the incompressible limit (Goldstein \& Leib 1988, Goldstein \& Hultgren 1988) and the compressible regime with $\mathcal{O}(1)$ Mach numbers (Goldstein \& Leib 1989). The analysis was then extended to construct a composite theory uniformly valid for Mach numbers $0 \leq M \sim \mathcal{O}(1)$. Numerical studies revealed that the amplitude development may exhibit a rich variety of behaviours, including explosive growth, equilibration, algebraic growth and violent "chaotic" oscillation. The theory is able to describe the most remarkable phe- 
nomenon in mixing-layer transition, vortex roll-up. The compressible weakly nonlinear theory, though formally valid for $M=\mathcal{O}(1)$, is found to be a poor approximation for the majority of parameters of interest.

As an asymptotic approach pertinent to high Reynolds numbers, the composite theory represents a methodology that complements DNS (e.g. Sandham \& Reynolds 1991, Ragab \& Sheen 1992), which could currently be performed only at fairly moderate Reynolds numbers. Mixing layers at high Reynolds numbers typical of practical applications remain inaccessible to DNS, and the parabolised-stability-equations (PSE) have been employed instead (e.g. Day, Mansour \& Reynolds 2001). In comparison with PSE, the present approach is more analytically based, and computationally more efficient since it focuses on the critical layer where nonlinear activities take place. It has been shown to capture qualitative behaviours of nonlinear instability and transition of mixing layers at Reynolds numbers beyond the reach of DNS. We note that the present theory may be further extended by taking into account the non-parallelism effect (which is usually included in the PSE approach) so that quantitative predictions may eventually be possible (cf. Hultgren 1992). The resulting improved theory would be especially useful when an extensive parametric study is required for design purpose.

One of the main motivations for studying mixing-layer instability and transition is to understand mixing process, which is closely related to roll-up of vorticity and temperature. From their patterns predicted by the theory, we may infer how mixing may be affected by various parameters such as the Reynolds number $R$, the temperature ratio $\beta_{T}$ and compressibility measured by $M$. For example, our calculations suggest that mixing can be enhanced by increasing $R,\left|\beta_{T}-1\right|$, or a combination of both.

It should be pointed out that the above conclusions concerning mixing is of qualitative nature, inferred from hydrodynamic and thermodynamic characteristics of the flow. A full quantitative characterisation of mixing relies on consideration of transport of binary, or more realistically, of reactive species, in a vortical flow. Much of the previous work assumes simple idealized flow structures, such as pairs of strained point vortices (RomKedar, Leonard \& Wiggins 1990) and slightly perturbed regular cat's eye of Kelvin (Ngan \& Shepherd 1997), which do not fully represent developing vortices in true mixing layers. Since the present work has provided an effective framework of describing these vortices, it paves the way for investigating mixing in more realistic, yet numerically tractable, models. Applications in this context is part of our ongoing research.

The authors would like to thank the referees for their helpful suggestions, which help them improve the presentation of the paper.

\section{A Derivation of the amplitude equation in the $\overline{\mathcal{M}} \gg 1$ limit}

The equations governing $\widetilde{T}_{m}^{(j)}$ and $\widetilde{Q}_{m}^{(j)}(j=0,1,2)$ are

$$
\begin{gathered}
\left(\frac{\partial}{\partial \widetilde{\mathcal{X}}}+\mathrm{i} \bar{Y}\right) \widetilde{T}_{1}^{(0)}-\bar{\Lambda} \frac{\partial^{2} \widetilde{T}_{1}^{(0)}}{\partial \widetilde{Y}^{2}}=\operatorname{isgn}\left(T_{c}^{\prime}\right) \widetilde{A} \\
\frac{\partial \widetilde{T}_{0}^{(1)}}{\partial \widetilde{\mathcal{X}}}+\frac{\mathrm{i}}{2} \frac{\partial}{\partial \bar{Y}}\left(\widetilde{A}^{*} \widetilde{T}_{1}^{(0)}-\widetilde{A} \widetilde{T}_{-1}^{(0)}\right)-\bar{\Lambda} \frac{\partial^{2} \widetilde{T}_{0}^{(1)}}{\partial \bar{Y}^{2}}=0 \\
\left(\frac{\partial}{\partial \widetilde{\mathcal{X}}}+2 \mathrm{i} \bar{Y}\right) \widetilde{T}_{2}^{(1)}+\frac{\mathrm{i}}{2} \frac{\partial}{\partial \bar{Y}}\left(-\widetilde{A} \widetilde{T}_{1}^{(0)}\right)-\bar{\Lambda} \frac{\partial^{2} \widetilde{T}_{2}^{(1)}}{\partial \bar{Y}^{2}}=0
\end{gathered}
$$




$$
\begin{gathered}
\left(\frac{\partial}{\partial \widetilde{\mathcal{X}}}+\mathrm{i} \bar{Y}\right) \widetilde{T}_{1}^{(2)}+\frac{\mathrm{i}}{2} \frac{\partial}{\partial \bar{Y}}\left(\widetilde{A}^{*} \widetilde{T}_{2}^{(1)}-\widetilde{A} \widetilde{T}_{0}^{(1)}\right)-\bar{\Lambda} \frac{\partial^{2} \widetilde{T}_{1}^{(2)}}{\partial \bar{Y}^{2}}=-\sigma\left(\frac{\alpha c}{2} \frac{\mathrm{d}}{\mathrm{d} \widetilde{\mathcal{X}}}+\mathrm{i}\right) \widetilde{A} \\
\left(\frac{\partial}{\partial \widetilde{\mathcal{X}}}+\mathrm{i} \bar{Y}\right) \widetilde{Q}_{1}^{(0)}+\bar{\Lambda} \frac{\partial^{2}}{\partial \bar{Y}^{2}}\left(\sigma_{c} \widetilde{T}_{1}^{(0)}-\widetilde{Q}_{1}^{(0)}\right)=0 \\
\frac{\partial \widetilde{Q}_{0}^{(1)}}{\partial \widetilde{\mathcal{X}}}+\frac{\mathrm{i}}{2} \frac{\partial}{\partial \bar{Y}}\left(\widetilde{A}^{*}\left(\widetilde{Q}_{1}^{(0)}-\widetilde{T}_{1}^{(0)}\right)-\widetilde{A}\left(\widetilde{Q}_{-1}^{(0)}-\widetilde{T}_{-1}^{(0)}\right)\right)+\bar{\Lambda} \frac{\partial^{2}}{\partial \bar{Y}^{2}}\left(\sigma_{c} \widetilde{T}_{0}^{(1)}-\widetilde{Q}_{0}^{(1)}\right)=0 \\
\left(\frac{\partial}{\partial \widetilde{\mathcal{X}}}+2 \mathrm{i} \bar{Y}\right) \widetilde{Q}_{2}^{(1)}+\frac{\mathrm{i}}{2} \frac{\partial}{\partial \bar{Y}}\left(-\widetilde{A}\left(\widetilde{Q}_{1}^{(0)}-\widetilde{T}_{1}^{(0)}\right)\right)+\bar{\Lambda} \frac{\partial^{2}}{\partial \bar{Y}^{2}}\left(\sigma_{c} \widetilde{T}_{2}^{(1)}-\widetilde{Q}_{2}^{(1)}\right)=0 \\
\left(\frac{\partial}{\partial \widetilde{\mathcal{X}}}+\mathrm{i} \bar{Y}\right) \widetilde{Q}_{1}^{(2)}+\frac{\mathrm{i}}{2} \frac{\partial}{\partial \bar{Y}}\left(\widetilde{A}^{*}\left(\widetilde{Q}_{2}^{(1)}-\widetilde{T}_{2}^{(1)}\right)-\widetilde{A}\left(\widetilde{Q}_{0}^{(1)}-\widetilde{T}_{0}^{(1)}\right)\right) \\
+\bar{\Lambda} \frac{\partial^{2}}{\partial \bar{Y}^{2}}\left(\sigma_{c} \widetilde{T}_{1}^{(2)}-\widetilde{Q}_{1}^{(2)}\right)=(1+\sigma)\left(\frac{\alpha c}{2} \frac{\mathrm{d} \widetilde{A}}{\mathrm{~d} \widetilde{\mathcal{X}}}+\mathrm{i} \widetilde{A}\right)
\end{gathered}
$$

while expansion of the jump condition (4.21) leads to

$$
\int_{-\infty}^{\infty} \widetilde{Q}_{1}^{(2)}(\widetilde{\mathcal{X}}, \bar{Y}) \mathrm{d} \bar{Y}=-2 \mathrm{i} \frac{T_{c} U_{c}^{\prime 2}}{\alpha U_{c}^{\prime \prime \prime}}\left(\frac{\alpha c}{2} J_{1} \frac{\mathrm{d}}{\mathrm{d} \widetilde{\mathcal{X}}}+\mathrm{i} J_{2}\right) \widetilde{A}
$$

where we have used the fact that $\widetilde{T}_{-m}^{(n)}=\widetilde{T}_{m}^{(n) *}$ and $\widetilde{Q}_{-m}^{(n)}=\widetilde{Q}_{m}^{(n) *}$.

Equations (A.1)-(A.8) is solved in sequence by taking Fourier transform with respect to $\bar{Y}$. The full solutions are rather lengthy, and in the following, we shall write out only the relevant parts of the solutions which affect the velocity jump and hence the amplitude equation. These solutions in Fourier space, to be denoted by a subscript 'F', are

$$
\begin{gathered}
\left(\widetilde{T}_{1}^{(0) *}\right)_{F}=-2 \operatorname{sgn}\left(T_{c}^{\prime}\right) \pi \mathrm{ie}^{-\frac{1}{3} \bar{\Lambda} \tilde{s}^{3}} \widetilde{A}^{*}(\widetilde{\mathcal{X}}-\tilde{s}) H(\tilde{s}), \\
\widetilde{T}_{0, F}^{(1)}=\operatorname{sgn}\left(T_{c}^{\prime}\right) \pi \mathrm{i} \tilde{s} \int_{0}^{\infty} \mathrm{e}^{-\frac{1}{3} \bar{\Lambda} \tilde{s}^{2}(\tilde{s}+3 \lambda)} \widetilde{A}(\widetilde{\mathcal{X}}-\lambda) \widetilde{A}^{*}(\tilde{\mathcal{X}}-\tilde{s}-\lambda) H(\tilde{s}) \mathrm{d} \lambda \\
\widetilde{T}_{1, F}^{(2)}=-\operatorname{sgn}\left(T_{c}^{\prime}\right) \frac{\pi \mathrm{i}}{2} \int_{0}^{\infty} \mathrm{e}^{-\frac{1}{3} \bar{\Lambda}\left(2(\tilde{s}+\lambda)^{3}-\tilde{s}^{3}\right)} \widetilde{A}(\widetilde{\mathcal{X}}-\lambda)(\tilde{s}+\lambda)^{2} H(\tilde{s}+\lambda) \\
\times \int_{0}^{\infty} \mathrm{e}^{-\bar{\Lambda}(\tilde{s}+\lambda)^{2} \mu} \widetilde{A}(\widetilde{\mathcal{X}}-\lambda-\mu) \widetilde{A}^{*}(\widetilde{\mathcal{X}}-\tilde{s}-2 \lambda-\mu) \mathrm{d} \mu \mathrm{d} \lambda, \\
\left(\widetilde{Q}_{1}^{(0) *}\right)_{F}=-\operatorname{sgn}\left(T_{c}^{\prime}\right) \frac{2 \pi \mathrm{i}}{3} \bar{\Lambda} \sigma_{c} \tilde{s}^{3} \mathrm{e}^{-\frac{1}{3} \bar{\Lambda} \tilde{s}^{3}} H(\tilde{s}) \widetilde{A}^{*}(\tilde{\mathcal{X}}-\tilde{s}), \\
\widetilde{Q}_{0, F}^{(1)}=\operatorname{sgn}\left(T_{c}^{\prime}\right) \pi \mathrm{i} \tilde{s} \int_{0}^{\infty} \mathrm{e}^{-\frac{1}{3} \bar{\Lambda} \sigma_{c} \tilde{s}^{2}(\tilde{s}+3 \lambda)} \widetilde{A}(\widetilde{\mathcal{X}}-\lambda) \widetilde{A}^{*}(\tilde{\mathcal{X}}-\tilde{s}-\lambda) H(\tilde{s}) \\
\times\left\{\frac{1}{3} \bar{\Lambda} \sigma_{c} \tilde{s}^{2}(\tilde{s}+3 \lambda)-1\right\} \mathrm{d} \lambda
\end{gathered}
$$




$$
\begin{aligned}
\widetilde{Q}_{1, F}^{(2)}= & 2 \pi \mathrm{e}^{\frac{1}{3} \bar{\Lambda} \tilde{s}^{3}} H(-\tilde{s})(1+\sigma)\left(\frac{\alpha c}{2} \frac{\mathrm{d}}{\mathrm{d} \widetilde{\mathcal{X}}}+\mathrm{i} \widetilde{\Omega}\right) \widetilde{A}(\widetilde{\mathcal{X}}+\tilde{s}) \\
+ & \operatorname{sgn}\left(T_{c}^{\prime}\right) \pi \mathrm{i} \int_{0}^{\infty}(\tilde{s}+\lambda)^{2} \mathrm{e}^{-\frac{1}{3} \bar{\Lambda}\left((\tilde{s}+\lambda)^{3}-\tilde{s}^{3}\right)} \\
& \times\left\{\int_{0}^{\infty}\left[1-\frac{1}{6} \bar{\Lambda} \sigma_{c}(\tilde{s}+\lambda)^{2}(\tilde{s}+\lambda+3 \mu)\right] \mathrm{e}^{-\frac{1}{3} \bar{\Lambda}(\tilde{s}+\lambda)^{2}(\tilde{s}+\lambda+3 \mu)}\right. \\
& \times \widetilde{A}(\widetilde{\mathcal{X}}-\lambda) \widetilde{A}(\widetilde{\mathcal{X}}-\lambda-\mu) \widetilde{A}^{*}(\widetilde{\mathcal{X}}-\tilde{s}-2 \lambda-\mu) H(\tilde{s}+\lambda) \mathrm{d} \mu \\
& -\frac{1}{2} \bar{\Lambda} \sigma_{c} \int_{0}^{\infty} \int_{0}^{\infty}(\tilde{s}+\lambda+\mu)^{2} \mathrm{e}^{-\frac{1}{3} \bar{\Lambda}\left(2(\tilde{s}+\lambda+\mu)^{3}-(\tilde{s}+\lambda)^{3}\right)} \widetilde{A}(\tilde{\mathcal{X}}-\lambda-\mu) H(\tilde{s}+\lambda+\mu) \\
& \left.\quad \times \mathrm{e}^{-\bar{\Lambda}(\tilde{s}+\lambda+\mu)^{2} \nu} \widetilde{A}(\widetilde{\mathcal{X}}-\lambda-\mu-\nu) \widetilde{A}^{*}(\widetilde{\mathcal{X}}-\tilde{s}-2 \lambda-2 \mu-\nu) \mathrm{d} \nu \mathrm{d} \mu\right\} \mathrm{d} \lambda,(\mathrm{A} .1)
\end{aligned}
$$

where $H$ is the Heaviside step function.

Evaluating the right-hand side of (A.9) by using (A.15) and the fact that

$$
\int_{-\infty}^{\infty} \widetilde{Q}_{1}^{(2)} \mathrm{d} \bar{Y}=\widetilde{Q}_{1, F}^{(2)}(\widetilde{\mathcal{X}}, \tilde{s}=0),
$$

we obtain the desired amplitude equation (4.30).

\section{References}

[1] BLUMEN, W. 1970 Shear layer instability of an inviscid compressible fluid. J. Fluid Mech. 40, 769-781.

[2] BLUMEN, W., DRAZIN, P.G. \& BILLINGS, D.F. 1975 Shear layer instability of an inviscid compressible fluid. Part 2. J. Fluid Mech. 71, 305-316.

[3] BROADWELL, J.E. \& BREIDENTHAL, R.E. 1982 A simple model of mixing and chemical reaction in a turbulent shear layer. J. Fluid Mech. 125, 397-410.

[4] BROWN, G.L. \& ROSHKO, A. 1974 On density effects and large structure in turbulent mixing layers. J. Fluid Mech. 64, 775-816.

[5] CLEMENS, N.T. \& MUNGAL, M.G. 1995 Large-scale structure and entrainment in the supersonic mixing layer. J. Fluid Mech. 284, 171-216.

[6] CURRAN, E.T., HEISER, W.H. \& PRATT, D.T. 1996 Fluid phenomena in scramjet combustion systems. Ann. Rev. Fluid Mech. 28, 323-360.

[7] COWLEY, S.J. \& WU, X. 1994 Asymptotic approaches to transition modelling. In Progress in Transition Modelling, AGARD Report 793, Chapter 3, 1-38.

[8] DAY, M.J., MANSOUR, N.N. \& REYNOLDS, W.C. 2001 Nonlinear stability and structure of compressible reacting mixing layers. J. Fluid Mech. 446, 375-408.

[9] DRAZIN, P.G. \& DAVEY, A. 1977 Shear layer instability of an inviscid compressible fluid. Part 3. J. Fluid Mech. 82, 255-260. 
[10] ELliOTT, G.S., SAMIMY, M. \& ARNETTE, S.A. 1995 The characteristics and evolution of large-scale structures in compressible mixing layers. Phys. Fluids 7, 864876.

[11] GOLDSTEIN, M.E. 1994 Nonlinear interactions between oblique instability waves on nearly parallel shear flows. Phys. Fluids A6, 724-735.

[12] GOLDSTEIN, M.E. \& HULTGREN, L.S. 1988 Nonlinear spatial evolution of an externally excited instability wave in a free shear layer. J. Fluid Mech. 197, 295-330.

[13] GOLDSTEIN, M.E. \& LEIB, S.J. 1988 Nonlinear roll-up of externally excited free shear layers. J. Fluid Mech. 191, 481-515.

[14] GOLDSTEIN, M.E. \& LEIB, S.J. 1989 Nonlinear evolution of oblique waves on compressible shear layers. J. Fluid Mech. 207, 73-96.

[15] GOLDSTEIN, M.E. \& WUNDROW, D.W. 1990 Spatial evolution of nonlinear acoustic mode instabilities on hypersonic boundary layers. J. Fluid Mech. 219, 585-607.

[16] GROSCH, C.E. \& JACKSON, T.L. 1991 Inviscid spatial stability of a threedimensional compressible mixing layer. J. Fluid Mech. 231, 35-50.

[17] GUTMARK, E.J., SCHADOW, K.C. \& YU, K.H. 1995 Mixing enhancement in supersonic free shear flows. Ann. Rev. Fluid Mech. 27, 375-417.

[18] HO, C. \& HUERRE, P. 1984 Perturbed free shear layers. Ann. Rev. Fluid Mech. 16, $365-422$.

[19] HULTGREN, L.S. 1992 Nonlinear spatial equilibration of an externally excited instability wave in a free shear layer. J. Fluid Mech. 236, 635-664.

[20] HUERRE, P. \& MONKEWITZ, P.A. 1990 Local and global instabilities in spatially developing flows. Ann. Rev. Fluid Mech. 22, 473-537.

[21] JACKSON, T.L. \& GROSCH, C.E. 1989 Inviscid spatial stability of a compressible mixing layer. J. Fluid Mech. 208, 609-637.

[22] JACKSON, T.L. \& GROSCH, C.E. 1991 Inviscid spatial stability of a compressible mixing layer. Part 3. Effect of thermodynamics. J. Fluid Mech. 224, 159-175.

[23] LEES, L. \& LIN, C.C. 1946 Investigation of the stability of the laminar boundary layer in a compressible fluid. NACA $T N-1115$

[24] LESSEN, M., FOX, J.A. \& ZIEN, H.M. 1965 On the inviscid stability of the laminar mixing of two parallel streams of a compressible fluid. J. Fluid Mech. 23, 355-367.

[25] LESSEN, M., FOX, J.A. \& ZIEN, H.M. 1966 Stability of the laminar mixing of two parallel streams with respect to supersonic disturbances. J. Fluid Mech. 25, 737-742.

[26] LEIB, S.J. 1991 Nonlinear evolution of subsonic and supersonic disturbances on a compressible free shear layer. J. Fluid Mech. 224, 551-578.

[27] LIU, J.T.C. 1989 Coherent structures in transitional and turbulent free shear flows. Ann. Rev. Fluid Mech. 21, 285-315.

[28] LOCK, R.C. 1951 The velocity distribution in the laminar boundary layer between parallel streams. Quart. J. Mech. 4, 42-63.

[29] MACK, L.M. 1984 Boundary layer linear instability theory. In Special Course on Stability and Transition of Laminar Flow, AGARD Rep. R-709, pp. 3-1-3-81.

[30] NGAN, K. \& SHEPHERD, T. 1997 Chaotic mixing and transport in Rossby-wave critical layers. J. Fluid Mech. 334, 315-351. 
[31] OLSEN, M.G. \& DUTTON, J.C. 2003 Planar velocity measurements in a weakly compressible mixing layer. J. Fluid Mech. 486, 51-77.

[32] RAGAB, S.A. \& SHEEN, S. 1992 The nonlinear development of supersonic instability waves in a mixing layer. Phys. Fluids A 4, 553-566.

[33] ROM-KEDAR, V., LEONARD, A. \& WIGGINS, S. 1990 An analytic study of transport, mixing and chaos in an unsteady vortical flow. J. Fluid Mech. 214, 347-394.

[34] SANDHAM, N. \& REYNOLDS, W.C. 1991 Three-dimensional simulations of a compressible mixing layer. J. Fluid Mech. 224, 133-158.

[35] SCHLICHTING, H. \& GERSTEN, K. 2000 Boundary-Layer Theory. Springer.

[36] SPARKS, C.A. 2006 Nonlinear instability of compressible mixing layers. PhD thesis, University of London, UK.

[37] STEWARTSON, K. 1964 The Theory of Laminar Boundary Layers in Compressible Fluids. Oxford University Press.

[38] STUART, J.T. 1960 On the non-linear mechanics of wave disturbances in stable and unstable parallel flows. Part 1. J. Fluid Mech. 9, 353-370.

[39] URBAN, W.D. \& MUNGAL, M.G. 2001 Planar velocity measurements in compressible mixing layers. J. Fluid Mech. 431, 189-222.

[40] WINANT, C.D. \& BROWAND, F.K. 1974 Vortex pairing: the mechanism of turbulent mixing-layer growth at moderate Reynolds number. J. Fluid Mech. 63, 237-255. 